UNIVERSIDAD POLITÉCNICA DE VALENCIA DEPARTAMENTO DE MATEMÁTICA APLICADA

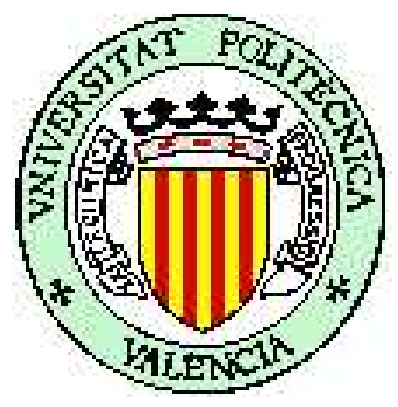

\title{
FUNCIONES ESPECIALES \\ Y ECUACIONES \\ DIFERENCIALES \\ MATRICIALES
}

TESIS DOCTORAL

Presentada por:

Juan Carlos Cortés López

Dirigida por:

Dr.D. Lucas Jódar Sánchez

Dr.D. José Luis Morera Fos

Valencia, Octubre de 1997 


\section{Índice general}

I MOTIVACION Y PRELIMINARES 1

1. MOTIVACION Y PRELIMINARES 2

II LAS FUNCIONES BETA, GAMMA E HIPERGEOMETRICA MATRICIALES 4

2. SOBRE ALGUNAS PROPIEDADES DE LAS FUNCIONES BETA Y GAMMA MATRICIALES 5

2.1. Introducción . . . . . . . . . . . . . . 5

2.2. Sobre las funciones matriciales Beta y Gamma . . . . . 7

3. SOBRE LA FUNCION Y LA ECUACION DIFERENCIAL HIPERGEOMETRICA MATRICIAL 29

3.1. Introducción . . . . . . . . . . . . . . . . . . . 29

3.2. Ecuaciones diferenciales matriciales lineales de segundo orden bilaterales . . . . . . . . . . . . . . 31

3.3. La función hipergeométrica matricial . . . . . . . . . . 34

3.4. La ecuación diferencial hipergeométrica matricial . . . . . . 41

3.5. Estudio de la función hipergeométrica matricial en la frontera de su recinto de convergencia . . . . . . . . . . . 48

3.6. Una representación integral para la función hipergeométrica matricial . . . . . . . . . . . . . . 53

\section{LAS ECUACIONES DIFERENCIALES MATRI-}


4. SOBRE LA ECUACION DIFERENCIAL MATRICIAL DE SYLVESTER CON COEFICIENTES VARIABLES $\quad \mathbf{6 4}$

4.1. Introducción . . . . . . . . . . . . . . . . 64

4.2. La convergencia de la serie solución . . . . . . . . . . 65

4.3. Lemas técnicos . . . . . . . . . . . . . . . . . 69

4.4. Construcción de aproximaciones precisas . . . . . . . 71

5. SOBRE LA ECUACION DIFERENCIAL MATRICIAL NO SIMETRICA DE RICCATI CON COEFICIENTES ANALITICOS 82

5.1. Introducción . . . . . . . . . . . . . . . 82

5.2. Lemas técnicos . . . . . . . . . . . . . . . 86

5.3. Error de truncación . . . . . . . . . . . . . . . . . 94

5.4. Sobre la estabilidad del método . . . . . . . . . . . . 105

IV BIBLIOGRAFIA 


\section{Parte I}

\section{MOTIVACION Y PRELIMINARES}




\section{Capítulo 1}

\section{MOTIVACION Y PRELIMINARES}

Este proyecto de tesis trata dos tipos de problemas relacionados con ciertas clases de ecuaciones diferenciales matriciales, como son la ecuación hipergeométrica matricial

$$
z(1-z) W^{\prime \prime}(z)-z A W^{\prime}(z)+W^{\prime}(z)(C-z(B+I))-A W(z) B=0,
$$

y la ecuación de Riccati con coeficientes matriciales variables

$$
W^{\prime}(t)=C(t)-D(t) W(t)-W(t) A(t)-W(t) B(t) W(t), \quad W(0)=W_{0} .
$$

El elemento unificador de la memoria es el método de Fröbenius matricial, que ya ha sido utilizado en las tesis doctorales de M. Legua [46], R. Company [9] y M. V. Ferrer [17]. La aportación más novedosa de esta memoria radica en la acotación del error de truncación de las soluciones en serie obtenidas, lo que permite obtener dos consecuencias de enorme interés en las aplicaciones, como son:

- La obtención de soluciones computables en forma finita.

- La construcción de soluciones aproximadas con una precisión prefijada.

Cabe decir que, por la información que tenemos, el análisis del error de truncación en términos de una precisión fijada de antemano, no está disponible en la literatura existente. 
En relación con la ecuación hipergeométrica matricial se trata en primer lugar de obtener un par de soluciones que permitan describir la solución general de (1.1) en términos de las mismas, sin considerar el problema ampliado equivalente. Se estudia también el error de truncación, cuando se obtiene la solución en serie de un problema de valores iniciales para (1.1), así como una representación integral de la función hipergeométrica matricial en términos de la función Gamma matricial.

El interés de la ecuación hipergeométrica es por una parte continuación de la emergente teoría de polinomios ortogonales matriciales, ya que en la evaluación de los coeficientes de los desarrollos en serie de polinomios ortogonales, aquéllos aparecen expresados en términos de la función hipergeométrica.

La ecuación de Riccati es una de las más estudiadas por su aparición en problemas clásicos y modernos de teoría de control, así como en la solución de problemas de contorno para sistemas lineales (véanse las referencias citadas en el capítulo dedicado a la ecuación de Riccati).

La clasificación temática de esta memoria atendiendo a los criterios de la 1991 AMS (MOS) subject classification es: 34A05, 34A25, 34A50, 41A20, 41A30, 47A60. 


\section{Parte II}

\section{LAS FUNCIONES BETA, GAMMA E HIPERGEOMETRICA MATRICIALES}




\section{Capítulo 2}

\section{SOBRE ALGUNAS PROPIEDADES DE LAS FUNCIONES BETA Y GAMMA MATRICIALES}

\subsection{Introducción}

Es bien sabido, que muchas de las funciones especiales ordinarias de la física-matemática, así como gran parte de sus propiedades, pueden deducirse a partir de la teoría de representación de grupos. También, en el estudio de funciones esféricas sobre ciertos espacios simétricos, así como en el análisis multivariante en Estadística aparecen funciones especiales de un argumento matricial, ver [28]. Asímismo, en [10] se utilizaron funciones especiales con dos argumentos matriciales diagonales. Recientemente, en el trabajo [33], sobre polinomios matriciales ortogonales se han utilizado funciones Beta con dos argumentos matriciales, pero en el que uno de dichos argumentos era un múltiplo escalar de la identidad.

En este capítulo, se demuestran algunas propiedades de las funciones matriciales Beta y Gamma que necesitaremos para estudiar en el siguiente capítulo la función hipergeométrica matricial. Concretamente, se da una expresión de la función Gamma matricial a través de un límite. Asímismo, se 
establecen condiciones para que dadas dos matrices $P, Q$ en $C^{r \times r}, B(P, Q)$ esté bien definida y se verifiquen las relaciones

$$
\begin{gathered}
B(P, Q)=B(Q, P), \\
B(P, Q)=\Gamma(P) \Gamma(Q) \Gamma^{-1}(P+Q) .
\end{gathered}
$$

Para mayor claridad en la presentación de las ideas, recordaremos algunas propiedades del cálculo funcional de Riesz-Dunford, las cuales pueden encontrarse en [15] , [23] y [21].

Si $P$ es una matriz en $C^{r \times r}$ denotamos por $\|P\|$ su 2-norma, la cual está definida en [21, p.56].

El conjunto de todos los valores propios de la matriz $P$ lo denotaremos por $\sigma(P)$.

Si $P$ es una matriz en $C^{r \times r}$ tal que, $\operatorname{Re}(z)>0$ para todo valor propio $z$ de $P$, entonces $\Gamma(P)$ dada por

$$
\Gamma(P)=\int_{0}^{\infty} e^{-t} t^{P-I} d t, \quad t^{P-I}=\exp ((P-I) \ln t)
$$

está bien definida.

Como la inversa de la función Gamma denotada por $\Gamma^{-1}(z)=1 / \Gamma(z)$, es una función entera de variable compleja $z$, para cualquier matriz $P$ en $C^{r \times r}$, el cálculo funcional de Riesz-Dunford muestra que la imagen $\operatorname{de} \Gamma^{-1}(z)$ actuando sobre $P$, denotada por $\Gamma^{-1}(P)$, es una matriz que está bien definida (ver [15, capítulo 7]). Además, si $P$ es una matriz que verifica la siguiente condición

$$
P+n I \text { es invertible para cualquier entero } n \geq 0
$$

entonces, $\Gamma(P)$ es invertible, su inversa coincide con $\Gamma^{-1}(P)$, y

$$
P(P+I) \ldots(P+(n-1) I) \Gamma^{-1}(P+n I)=\Gamma^{-1}(P), \quad n \geq 1
$$

(ver [23, p.253]).

FUNC. ESPECIALES Y EC. DIF. MATRICIALES 
Si $f(z)$ y $g(z)$ son funciones holomorfas de variable compleja $z$, las cuales están definidas en un conjunto abierto $\Omega$ del plano complejo, y $P$ es una matriz en $C^{r \times r}$ tal que $\sigma(P) \subset \Omega$, entonces por las propiedades del cálculo funcional matricial [15, p.558], se deduce que $f(P) g(P)=g(P) f(P)$. Entonces, por ésto, bajo la condición (2.2), la ecuación (2.3) puede escribirse en la forma

$$
P(P+I) \ldots(P+(n-1) I)=\Gamma(P+n I) \Gamma^{-1}(P), \quad n \geq 1
$$

Si tenemos en cuenta que la función factorial escalar, denotada por $(z)_{n}$ y definida por $(z)_{n}=z(z+1) \ldots(z+n-1), \quad n \geq 1, \quad(z)_{0}=1$ es entera, entonces por aplicación del cálculo funcional matricial sobre esta función, se deduce que para cualquier matriz $P$ en $C^{r \times r}$ se cumple

$$
(P)_{n}=P(P+I) \ldots(P+(n-1) I), \quad n \geq 1, \quad(P)_{0}=I
$$

Si $f(P)$ está bien definida y $S$ es una matriz invertible en $C^{r \times r}$, entonces por [21, p.541]

$$
f\left(S P S^{-1}\right)=S f(P) S^{-1}
$$

Si $P$ varía en $C^{r \times r}$, usando su descomposición de Schur y denotando por $\alpha(P)=\operatorname{máx}_{z \in \sigma(P)}\{\operatorname{Re}(z)\}$ para $t \in R$ se deduce por [21, p.336, 556] que

$$
\left\|e^{t P}\right\| \leq e^{t \alpha(P)} \sum_{k=0}^{r-1} \frac{(\|P\| \sqrt{r} t)^{k}}{k !}
$$

\subsection{Sobre las funciones matriciales Beta y Gam- ma}

Sea $M$ una matriz en $C^{r \times r}$ tal que

$$
\operatorname{Re}(z)>0 \text { para todo } z \text { en } \sigma(M)
$$

y sea $n$ un entero, $n \geq 1$. Usando la técnica de integración por partes para obtener primero una fórmula de reducción, es sencillo deducir tal y como puede verse en [52, p.17] que

$$
g(z)=\int_{0}^{1}(1-s)^{n} s^{z-1} d s=n ![z(z+1) \ldots(z+n)]^{-1}, \quad \operatorname{Re}(z)>0
$$

FUNC. ESPECIALES Y EC. DIF. MATRICIALES 
y de aquí deducimos

$$
f(z)=\int_{0}^{n}\left(1-\frac{s}{n}\right)^{n} s^{z-1} d s=n ! n^{z}[z(z+1) \ldots(z+n)]^{-1}, \quad \operatorname{Re}(z)>0
$$

Como $f$ y $g$ son funciones holomorfas en $\operatorname{Re}(z)>0$, por aplicación del cálculo funcional matricial en (2.9) y (2.10) se tiene

$$
\begin{gathered}
g(M)=\int_{0}^{1}(1-s)^{n} s^{M-I} d s=n ![M(M+I) \ldots(M+n I)]^{-1} \\
f(M)=\int_{0}^{n}\left(1-\frac{s}{n}\right)^{n} s^{M-I} d s=n ! n^{M}[M(M+I) \ldots(M+n I)]^{-1} .
\end{gathered}
$$

Por (2.1) y (2.12) podemos escribir

$$
\begin{gathered}
\Gamma(M)-n ! n^{M}[M(M+I) \ldots(M+n I)]^{-1} \\
=\int_{0}^{\infty} e^{-t} t^{M-I} d t-\int_{0}^{n}\left(1-\frac{t}{n}\right)^{n} t^{M-I} d t \\
=\int_{0}^{n}\left[e^{-t}-\left(1-\frac{t}{n}\right)^{n}\right] t^{M-I} d t+\int_{n}^{\infty} e^{-t} t^{M-I} d t
\end{gathered}
$$

Como $\int_{0}^{\infty} e^{-t} t^{M-I} d t$ es convergente, se tiene

$$
\lim _{n \rightarrow \infty} \int_{n}^{\infty} e^{-t} t^{M-I} d t=0
$$

Ahora probamos que

$$
\lim _{n \rightarrow \infty} \int_{0}^{n}\left[e^{-t}-\left(1-\frac{t}{n}\right)^{n}\right] t^{M-I} d t=0
$$

Por [52, p.16] sabemos

$$
0 \leq e^{-t}-\left(1-\frac{t}{n}\right)^{n} \leq \frac{t^{2} e^{-t}}{n}, \quad 0 \leq t<n
$$

luego

$$
\left\|\int_{0}^{n}\left[e^{-t}-\left(1-\frac{t}{n}\right)^{n}\right] t^{M-I} d t\right\| \leq \frac{1}{n} \int_{0}^{n}\left\|t^{M+I}\right\| e^{-t} d t
$$


Por (2.7) y usando que $\ln t \leq t$ para $t>0$, podemos escribir

$$
\begin{gathered}
\left\|t^{M+I}\right\| \leq t^{\alpha(M)+1} \sum_{j=0}^{r-1} \frac{[(\|M\|+1) \sqrt{r} \ln t]^{j}}{j !} \\
\leq t^{\alpha(M)+1} \sum_{j=0}^{r-1} \frac{[(\|M\|+1) \sqrt{r} t]^{j}}{j !}, \quad t>0
\end{gathered}
$$

Por (2.16) y (2.17),

$$
\frac{1}{n} \int_{0}^{n}\left\|t^{M+I}\right\| e^{-t} d t \leq \frac{1}{n}\left\{\sum_{j=0}^{r-1} \frac{[(\|M\|+1) \sqrt{r}]^{j}}{j !} \int_{0}^{n} e^{-t} t^{\alpha(M)+1+j} d t\right\}
$$

Como para $0 \leq j \leq r-1$, tenemos

$$
\int_{0}^{\infty} e^{-t} t^{\alpha(M)+1+j} d t<+\infty
$$

por (2.16)-(2.19) se tiene (2.15).

Por tanto acabamos de establecer el siguiente resultado:

Teorema 2.2.1. Sea $M$ una matriz que satisface (2.8) y sea $n \geq 1$ un entero. Entonces

$$
\Gamma(M)=\lim _{n \rightarrow \infty}(n-1) !(M)_{n}^{-1} n^{M},
$$

donde $(M)_{n}$ está definida por (2.5).

Sean $P, Q$ matrices en $C^{r \times r}$ tales que

$$
\operatorname{Re}(z)>0, \operatorname{Re}(w)>0 \text { para todo } z \in \sigma(P), \quad w \in \sigma(Q)
$$

Por (2.7) y usando que $\ln t<t$ y $\ln (1-t)<1-t$ para $0<t<1$, se 
deduce que

$$
\begin{gathered}
\int_{0}^{1}\left\|t^{P-I}\right\|\left\|(1-t)^{Q-I}\right\| d t \\
\leq \sum_{j=0}^{r-1} \sum_{k=0}^{r-1} \frac{(\|P\|+1)^{j}(\|Q\|+1)^{k}(\sqrt{r})^{j+k}}{j ! k !} \int_{0}^{1} t^{\alpha(P)-1}(1-t)^{\alpha(Q)-1} \ln ^{j}(t) \ln ^{k}(1-t) d t \\
\leq \sum_{j=0}^{r-1} \sum_{k=0}^{r-1} \frac{(\|P\|+1)^{j}(\|Q\|+1)^{k} r^{\frac{j+k}{2}}}{j ! k !} \int_{0}^{1} t^{\alpha(P)+j-1}(1-t)^{\alpha(Q)+k-1} d t \\
=\sum_{j=0}^{r-1} \sum_{k=0}^{r-1} \frac{(\|P\|+1)^{j}(\|Q\|+1)^{k} r^{\frac{j+k}{2}} B(\alpha(P)+j, \alpha(Q)+k)}{j ! k !}<+\infty
\end{gathered}
$$

Luego podemos definir

$$
B(P, Q)=\int_{0}^{1} t^{P-I}(1-t)^{Q-I} d t
$$

En [33] se muestra que si $P, Q$ son matrices en $C^{r \times r}$ satisfaciendo (2.20) y $P$ ó $Q$ son múltiplos escalares de la matriz identidad, entonces se verifica que $B(P, Q)=B(Q, P)$.

El siguiente ejemplo muestra que si $P$ o $Q$ no conmutan, entonces la propiedad $B(P, Q)=B(Q, P)$ no es cierta.

\section{Ejemplo 1. Sean}

$$
P=\left[\begin{array}{ll}
1 & 0 \\
1 & 2
\end{array}\right], \quad Q=\left[\begin{array}{ll}
1 & 1 \\
0 & 2
\end{array}\right]
$$

matrices en $C^{2 \times 2}$ para las cuales se tiene que $\sigma(P)=\sigma(Q)=\{1,2\}$. Entonces se cumple que ambas matrices no conmutan pues

$$
P Q=\left[\begin{array}{ll}
1 & 1 \\
1 & 5
\end{array}\right] \neq\left[\begin{array}{ll}
2 & 2 \\
2 & 4
\end{array}\right]=Q P .
$$

Notar que $\left[\begin{array}{ll}0 & 0 \\ 1 & 1\end{array}\right]^{n}=\left[\begin{array}{ll}0 & 0 \\ 1 & 1\end{array}\right]$ y $\left[\begin{array}{ll}0 & 1 \\ 0 & 1\end{array}\right]^{n}=\left[\begin{array}{ll}0 & 1 \\ 0 & 1\end{array}\right]$ para todo $n \geq 1$. 
Luego para $0<t<1$ se tiene

$t^{P-I}=t\left[\begin{array}{ll}0 & 0 \\ 1 & 1\end{array}\right]=\left[\begin{array}{cc}1 & 0 \\ t-1 & t\end{array}\right] ; \quad(1-t)^{Q-I}=(1-t)\left[\begin{array}{ll}0 & 1 \\ 0 & 1\end{array}\right]=\left[\begin{array}{cc}1 & -t \\ 0 & 1-t\end{array}\right]$

$\mathrm{y}$

$(1-t)^{P-I}=(1-t)\left[\begin{array}{ll}0 & 0 \\ 1 & 1\end{array}\right]=\left[\begin{array}{cc}1 & 0 \\ -t & 1-t\end{array}\right] ; t^{Q-I}=t\left[\begin{array}{ll}0 & 1 \\ 0 & 1\end{array}\right]=\left[\begin{array}{cc}1 & t-1 \\ 0 & t\end{array}\right]$

Luego

$$
\begin{gathered}
B(P, Q)=\int_{0}^{1} t^{P-I}(1-t)^{Q-I} d t=\int_{0}^{1}\left[\begin{array}{cc}
1 & 0 \\
t-1 & t
\end{array}\right]\left[\begin{array}{cc}
1 & -t \\
0 & 1-t
\end{array}\right] d t \\
=\int_{0}^{1}\left[\begin{array}{cc}
1 & -t \\
t-1 & 2 t(1-t)
\end{array}\right] d t=\left[\begin{array}{cc}
1 & -1 / 2 \\
-1 / 2 & 1 / 3
\end{array}\right]
\end{gathered}
$$

$\mathrm{y}$

$$
\begin{gathered}
B(Q, P)=\int_{0}^{1} t^{Q-I}(1-t)^{P-I} d t=\int_{0}^{1}\left[\begin{array}{cc}
1 & t-1 \\
0 & t
\end{array}\right]\left[\begin{array}{cc}
1 & 0 \\
-t & 1-t
\end{array}\right] d t \\
\quad=\int_{0}^{1}\left[\begin{array}{cc}
-t^{2}+t+1 & -(1-t)^{2} \\
-t^{2} & t(1-t)
\end{array}\right] d t=\left[\begin{array}{cc}
7 / 6 & -1 / 3 \\
-1 / 3 & 1 / 6
\end{array}\right]
\end{gathered}
$$

Por tanto $B(P, Q) \neq B(Q, P)$.

Los siguientes lemas son fáciles de probar, por lo que tan sólo demostraremos el primero.

Lema 2.2.1 . Sean $P, Q$ matrices que conmutan en $C^{r \times r}$, satisfaciendo (2.20), entonces:

$$
B(P, Q)=B(Q, P)
$$


Demostración. Como $P Q=Q P$, se deduce

$$
(P-I)(\ln t)(Q-I) \ln (1-t)=(Q-I)(\ln (1-t))(P-I) \ln t
$$

para $0<t<1$. Luego podemos escribir

$$
\begin{gathered}
B(P, Q)=\int_{0}^{1} t^{P-I}(1-t)^{Q-I} d t \\
=\int_{0}^{1} e^{(P-I) \ln t} e^{(Q-I) \ln (1-t)} d t=\int_{0}^{1} e^{(Q-I) \ln (1-t)} e^{(P-I) \ln t} d t \\
=\int_{0}^{1} e^{(Q-I) \ln u} e^{(P-I) \ln (1-u)} d u=B(Q, P)
\end{gathered}
$$

Lema 2.2.2 . Sean D, E matrices diagonales satisfaciendo (2.20). Entonces

$$
B(D, E)=\Gamma(D) \Gamma(E) \Gamma^{-1}(D+E)
$$

Teorema 2.2.2 . Sean P, $Q$ matrices diagonalizables en $C^{r \times r}$ verificando que $P Q=Q P$ y que satisfacen la condición (2.20). Entonces

$$
B(P, Q)=\Gamma(P) \Gamma(Q) \Gamma^{-1}(P+Q)
$$

Demostración. Como $P, Q$ son diagonalizables y conmutan, por el teorema 1.3.12 de [24], se deduce que son simultáneamente diagonalizables. Sea $S$ una matriz invertible en $C^{r \times r}$ tal que

$$
S^{-1} P S=D, S^{-1} Q S=E ; \quad D, E \text { son matrices diagonales }
$$

Para demostrar (2.22) observar que por [24, p.54], si $\sigma(P)=\left\{\lambda_{1}, \ldots, \lambda_{r}\right\}$ y $\sigma(Q)=\left\{\mu_{1}, \ldots, \mu_{r}\right\}$, entonces

$$
\sigma(P+Q)=\left\{\lambda_{j}+\mu_{i_{j}}\right\}_{j=1}^{r}
$$

para alguna permutación $i_{1}, i_{2}, \ldots, i_{r}$ de $1,2, \ldots, r$.

Como las matrices P y Q satisfacen (2.20), se deduce que

$$
\operatorname{Re}(s)>0 \quad \text { para todo } s \in \sigma(P+Q)
$$


Por el lema 2.2.2, por (2.6) y por (2.23) se deduce

$$
P+Q=S(D+E) S^{-1}
$$

y

$$
\begin{gathered}
\Gamma(P+Q)=S\left[\int_{0}^{\infty} e^{-t} t^{D+E-I} d t\right] S^{-1}=S \Gamma(D+E) S^{-1}, \\
\Gamma(P)=S \Gamma(D) S^{-1}, \quad \Gamma(Q)=S \Gamma(E) S^{-1} \\
B(P, Q)=S B(D, E) S^{-1}=S\left[\Gamma(D) \Gamma(E) \Gamma^{-1}(D+E)\right] S^{-1},
\end{gathered}
$$

Por (2.25) se tiene $\Gamma^{-1}(D+E)=S^{-1} \Gamma^{-1}(P+Q) S$, y por (2.26) y (2.27) se sigue que

$$
\begin{gathered}
B(P, Q)=S \Gamma(D) \Gamma(E)\left[S^{-1} \Gamma^{-1}(P+Q) S\right] S^{-1} \\
=\left(S \Gamma(D) S^{-1}\right)\left(S \Gamma(E) S^{-1}\right) \Gamma^{-1}(P+Q) \\
=\Gamma(P) \Gamma(Q) \Gamma^{-1}(P+Q)
\end{gathered}
$$

Por tanto el resultado queda probado.

Nota 1. Además de la hipótesis de conmutatividad, la condición de diagonalización del teorema 2.2.2 garantiza que cualquier valor propio $z$ de la matriz $P+Q$ está en el semiplano derecho del plano complejo. El siguiente ejemplo muestra que en general si $P, Q$ son matrices satisfaciendo (2.20) su suma $P+Q$ no satisface esta condición.

Sean $a$ y $b$ números positivos tales que $a b>1$. Entonces las matrices

$$
P=\left[\begin{array}{cc}
1 / 2 & 0 \\
a & 1 / 2
\end{array}\right], \quad Q=\left[\begin{array}{cc}
1 / 2 & b \\
0 & 1 / 2
\end{array}\right]
$$

FUNC. ESPECIALES Y EC. DIF. MATRICIALES 
cumplen que $\sigma(P)=\sigma(Q)=\{1 / 2\}$, pero

$$
P+Q=\left[\begin{array}{ll}
1 & b \\
a & 1
\end{array}\right]
$$

y $\sigma(P+Q)=\{1-\sqrt{a b}, 1+\sqrt{a b}\}$ con $1-\sqrt{a b}<0$.

Ejemplo 2. Sean

$$
P=\left[\begin{array}{ll}
1 & 1 \\
0 & 1
\end{array}\right], \quad Q=\left[\begin{array}{ll}
1 & 0 \\
0 & 2
\end{array}\right] .
$$

Entonces, $\sigma(P)=\{1\}, \sigma(Q)=\{1,2\} \mathrm{y}$

$$
P Q=\left[\begin{array}{ll}
1 & 2 \\
0 & 2
\end{array}\right] \neq\left[\begin{array}{ll}
1 & 1 \\
0 & 2
\end{array}\right]=Q P
$$

Notar que: $P-I=\left[\begin{array}{ll}0 & 1 \\ 0 & 0\end{array}\right]$ y $\left[\begin{array}{ll}0 & 1 \\ 0 & 0\end{array}\right]^{2}=0 \in C^{2 \times 2}$. Luego para $t>0$ se tiene

$$
t^{P-I}=\exp \left(\left[\begin{array}{ll}
0 & 1 \\
0 & 0
\end{array}\right] \ln t\right)=\left[\begin{array}{ll}
1 & 0 \\
0 & 1
\end{array}\right]+\left[\begin{array}{ll}
0 & 1 \\
0 & 0
\end{array}\right] \ln t=\left[\begin{array}{cc}
1 & \ln t \\
0 & 1
\end{array}\right] .
$$

Además, $Q-I=\left[\begin{array}{ll}0 & 0 \\ 0 & 1\end{array}\right]$ y $\left[\begin{array}{ll}0 & 0 \\ 0 & 1\end{array}\right]^{k}=\left[\begin{array}{ll}0 & 0 \\ 0 & 1\end{array}\right]$ para $k \geq 1$. Luego, para $0<t<1$ se tiene

$$
\begin{gathered}
(1-t)^{Q-I}=\exp \left(\left[\begin{array}{ll}
0 & 0 \\
0 & 1
\end{array}\right] \ln (1-t)\right) \\
=\left[\begin{array}{ll}
1 & 0 \\
0 & 1
\end{array}\right]+\sum_{k \geq 1}\left[\begin{array}{ll}
0 & 0 \\
0 & 1
\end{array}\right] \frac{\ln ^{k}(1-t)}{k !}=\left[\begin{array}{cc}
1 & 0 \\
0 & 1-t
\end{array}\right],
\end{gathered}
$$

y

$$
t^{P-I}(1-t)^{Q-I}=\left[\begin{array}{cc}
1 & (1-t) \ln t \\
0 & 1-t
\end{array}\right]
$$


por tanto, como ya disponemos de todos los cálculos estamos en condiciones de poder evaluar la función Beta de argumentos matriciales $P$ y $Q$ dadas anteriormente

$B(P, Q)=\int_{0}^{1} t^{P-I}(1-t)^{Q-I} d t=\left[\begin{array}{cc}\int_{0}^{1} d t & \int_{0}^{1}(1-t) \ln t d t \\ 0 & \int_{0}^{1}(1-t) d t\end{array}\right]=\left[\begin{array}{cc}1 & -3 / 4 \\ 0 & 1 / 2\end{array}\right]$.

Ahora pasamos a calcular $B(Q, P)$.

De una forma análoga, para $0<t<1$ se tiene

$$
\begin{gathered}
t^{Q-I}=\exp \left(\left[\begin{array}{ll}
0 & 0 \\
0 & 1
\end{array}\right] \ln t\right)=\left[\begin{array}{ll}
1 & 0 \\
0 & t
\end{array}\right], \\
(1-t)^{P-I}=\exp \left(\left[\begin{array}{ll}
0 & 1 \\
0 & 0
\end{array}\right] \ln (1-t)\right)=\left[\begin{array}{cc}
1 & \ln (1-t) \\
0 & 1
\end{array}\right],
\end{gathered}
$$

$\mathrm{y}$

$$
B(Q, P)=\int_{0}^{1} t^{Q-I}(1-t)^{P-I} d t=\left[\begin{array}{cc}
\int_{0}^{1} d t & \int_{0}^{1} \ln (1-t) d t \\
0 & \int_{0}^{1} t d t
\end{array}\right]=\left[\begin{array}{cc}
1 & -1 \\
0 & 1 / 2
\end{array}\right] .
$$

Luego $B(P, Q) \neq B(Q, P)$. Además

$$
\begin{gathered}
\Gamma(P)=\int_{0}^{\infty} e^{-t} t^{P-I} d t=\int_{0}^{\infty} e^{-t}\left[\begin{array}{cc}
1 & \ln t \\
0 & 1
\end{array}\right] d t \\
=\int_{0}^{\infty}\left[\begin{array}{cc}
e^{-t} & e^{-t} \ln t \\
0 & e^{-t}
\end{array}\right] d t=\left[\begin{array}{cc}
1 & -C \\
0 & 1
\end{array}\right],
\end{gathered}
$$

donde $C=0,577215 \ldots$ es la constante de Euler-Mascheroni, y hemos utilizado, por $[22$, p.602] que

$$
\int_{0}^{\infty} e^{-t} \ln t d t=-C
$$


$\mathrm{y}$

$$
\begin{aligned}
& \Gamma(Q)=\int_{0}^{\infty} e^{-t} t^{Q-I} d t=\int_{0}^{\infty}\left[\begin{array}{cc}
e^{-t} & 0 \\
0 & t e^{-t}
\end{array}\right] d t=\left[\begin{array}{ll}
1 & 0 \\
0 & 1
\end{array}\right] \\
& P+Q-I=\left[\begin{array}{ll}
1 & 1 \\
0 & 2
\end{array}\right]=\left[\begin{array}{ll}
1 & 1 \\
0 & 1
\end{array}\right]\left[\begin{array}{ll}
1 & 0 \\
0 & 2
\end{array}\right]\left[\begin{array}{cc}
1 & -1 \\
0 & 1
\end{array}\right]
\end{aligned}
$$

luego

$$
\begin{gathered}
e^{-t} t\left[\begin{array}{ll}
1 & 1 \\
0 & 2
\end{array}\right]=\exp \left(-t\left[\begin{array}{ll}
1 & 0 \\
0 & 1
\end{array}\right]+\left[\begin{array}{ll}
1 & 1 \\
0 & 1
\end{array}\right]\left[\begin{array}{ll}
1 & 0 \\
0 & 2
\end{array}\right]\left[\begin{array}{rr}
1 & -1 \\
0 & 1
\end{array}\right] \ln t\right) \\
=\exp \left\{\left[\begin{array}{ll}
1 & 1 \\
0 & 1
\end{array}\right]\left(-t\left[\begin{array}{ll}
1 & 0 \\
0 & 1
\end{array}\right]+\left[\begin{array}{ll}
1 & 0 \\
0 & 2
\end{array}\right] \ln t\right)\left[\begin{array}{rr}
1 & -1 \\
0 & 1
\end{array}\right]\right\} \\
=\exp \left(\left[\begin{array}{ll}
1 & 1 \\
0 & 1
\end{array}\right]\left[\begin{array}{cc}
-t+\ln t & 0 \\
0 & -t+2 \ln t
\end{array}\right]\left[\begin{array}{rr}
1 & -1 \\
0 & 1
\end{array}\right]\right) \\
=\left[\begin{array}{ll}
1 & 1 \\
0 & 1
\end{array}\right] \exp \left(\left[\begin{array}{cc}
-t+\ln t & 0 \\
0 & -t+2 \ln t
\end{array}\right]\right)\left[\begin{array}{rr}
1 & -1 \\
0 & 1
\end{array}\right] \\
=\left[\begin{array}{cc}
t e^{-t} t^{2} e^{-t}-t e^{-t} \\
0 & t^{2} e^{-t}
\end{array}\right]
\end{gathered}
$$

Luego

$$
\Gamma(P+Q)=\int_{0}^{\infty} e^{-t} t^{P+Q-I} d t=\int_{0}^{\infty}\left[\begin{array}{cc}
t e^{-t} & t^{2} e^{-t}-t e^{-t} \\
0 & t^{2} e^{-t}
\end{array}\right] d t=\left[\begin{array}{ll}
1 & 1 \\
0 & 2
\end{array}\right]
$$

Por tanto

$$
B(P, Q)=\left[\begin{array}{cc}
1 & -3 / 4 \\
0 & 1 / 2
\end{array}\right] \neq \Gamma(P) \Gamma(Q) \Gamma^{-1}(P+Q)
$$


ya que

$$
\begin{gathered}
\Gamma(P) \Gamma(Q) \Gamma^{-1}(P+Q)=\left[\begin{array}{cc}
1 & -C \\
0 & 1
\end{array}\right]\left[\begin{array}{ll}
1 & 0 \\
0 & 1
\end{array}\right]\left[\begin{array}{ll}
1 & 1 \\
0 & 2
\end{array}\right]^{-1} \\
\quad=\frac{1}{2}\left[\begin{array}{cc}
1 & -C \\
0 & 1
\end{array}\right]\left[\begin{array}{cc}
2 & -1 \\
0 & 1
\end{array}\right]=\frac{1}{2}\left[\begin{array}{cc}
2 & -1-C \\
0 & 1
\end{array}\right] .
\end{gathered}
$$

luego en este caso, en que la matriz $P$ no es diagonalizable y $P Q \neq Q P$, el teorema 2.2.2 de este capítulo no es válido.

En el teorema 2.2.2 hemos establecido la fórmula

$$
B(P, Q)=\Gamma(P) \Gamma(Q) \Gamma^{-1}(P+Q)
$$

suponiendo que $P, Q$ son matrices diagonalizables en $C^{r \times r}$ que conmutan y verifican la condición (2.20). En lo que sigue estableceremos el mismo resultado a partir de hipótesis distintas, entre las cuales desaparece la condición de diagonalización de las matrices $P$ y $Q$. La idea de relajar las hipótesis del teorema 2.2.2 eliminando la condición de diagonalización de ambas matrices $P$ y $Q$, viene motivada por el siguiente ejemplo en el que aún no siendo una de las dos matrices diagonalizable, la fórmula (2.22) sigue siendo válida.

Ejemplo 3. Sean las matrices

$$
P=\left[\begin{array}{cc}
1 / 2 & 0 \\
0 & 1 / 2
\end{array}\right], \quad Q=\left[\begin{array}{cc}
1 / 2 & b \\
0 & 1 / 2
\end{array}\right] \quad b \neq 0 .
$$

Entonces se tiene que $P$ es diagonalizable y $\sigma(P)=\{1 / 2\}>0$. $Q$ no es diagonalizable y $\sigma(Q)=\{1 / 2\}>0$. Notar que $P$ y $Q$ conmutan,

$$
P Q=\left[\begin{array}{cc}
1 / 4 & b / 2 \\
0 & 1 / 4
\end{array}\right]=Q P
$$

Además,

$$
P+Q=\left[\begin{array}{ll}
1 & b \\
0 & 1
\end{array}\right]
$$

y $\sigma(P+Q)=\{1\}>0$.

FUNC. ESPECIALES Y EC. DIF. MATRICIALES 
Ahora desarrollando los cálculos que necesitamos, obtenemos

$$
t^{P-I}=t\left[\begin{array}{cc}
-1 / 2 & 0 \\
0 & -1 / 2
\end{array}\right]=\left[\begin{array}{cc}
t^{-1 / 2} & 0 \\
0 & t^{-1 / 2}
\end{array}\right]
$$

Luego

$$
\Gamma(P)=\int_{0}^{\infty} e^{-t} t^{P-I} d t=\int_{0}^{\infty}\left[\begin{array}{cc}
e^{-t} t^{-1 / 2} & 0 \\
0 & e^{-t} t^{-1 / 2}
\end{array}\right] d t=\left[\begin{array}{cc}
\sqrt{\pi} & 0 \\
0 & \sqrt{\pi}
\end{array}\right]
$$

donde hemos utilizado la conocida relación

$$
\int_{0}^{\infty} t^{\nu-1} e^{-\mu t} d t=\frac{\Gamma(\nu)}{\mu^{\nu}}
$$

aplicada para $\nu=1 / 2$ y $\mu=1$, y que $\Gamma(1 / 2)=\sqrt{\pi}$.

Como

$$
t^{Q-I}=t\left[\begin{array}{cc}
t^{-1 / 2} & b t^{-1 / 2} \ln t \\
0 & t^{-1 / 2}
\end{array}\right]
$$

se tiene

$$
\begin{gathered}
\Gamma(Q)=\int_{0}^{\infty} e^{-t} t^{Q-I} d t=\int_{0}^{\infty}\left[\begin{array}{cc}
e^{-t} t^{-1 / 2} & b e^{-t} t^{-1 / 2} \ln t \\
0 & e^{-t} t^{-1 / 2}
\end{array}\right] d t \\
=\left[\begin{array}{cc}
\sqrt{\pi} & -b \sqrt{\pi}(C+2 \ln 2) \\
0 & \sqrt{\pi}
\end{array}\right]
\end{gathered}
$$

donde $C$ como en el ejemplo anterior, denota la constante de Euler-Mascheroni. Observemos que en el último paso de los cálculos anteriores $\operatorname{para} \Gamma(Q)$ hemos utilizado, según [22, p.576] que

$$
\int_{0}^{\infty} t^{\nu-1} e^{-\mu t} \ln t d t=\frac{1}{\mu^{\nu}} \Gamma(\nu)[\psi(\nu)-\ln \mu], \quad \operatorname{Re}(\mu)>0, \quad \operatorname{Re}(\nu)>0
$$

siendo $\psi$ la función psi de Euler. En nuestro caso hemos aplicado la fórmula anterior para $\nu=1 / 2$ y $\mu=1$. Así obtenemos

$$
\int_{0}^{\infty} e^{-t} t^{-1 / 2} \ln t d t=\frac{1}{1^{1 / 2}} \Gamma(1 / 2)[\psi(1 / 2)-\ln 1]
$$

FUNC. ESPECIALES Y EC. DIF. MATRICIALES 
y ahora teniendo en cuenta que $\psi(1 / 2)=-C-2 \ln 2$ concluimos que

$$
\int_{0}^{\infty} e^{-t} t^{-1 / 2} \ln t d t=-(C+2 \ln 2) \sqrt{\pi}
$$

Calculamos ahora $t^{P+Q-I}$

$$
t^{P+Q-I}=t\left[\begin{array}{ll}
0 & b \\
0 & 0
\end{array}\right]=\left[\begin{array}{cc}
1 & b \ln t \\
0 & 1
\end{array}\right]
$$

luego

$$
\Gamma(P+Q)=\int_{0}^{\infty} e^{-t} t^{P+Q-I} d t=\int_{0}^{\infty}\left[\begin{array}{cc}
e^{-t} & b e^{-t} \ln t \\
0 & e^{-t}
\end{array}\right] d t=\left[\begin{array}{cc}
1 & -b C \\
0 & 1
\end{array}\right]
$$

Ahora calculamos $B(P, Q)$, para ello empezamos evaluando

$$
t^{P-I}(1-t)^{Q-I}=\left[\begin{array}{cc}
t^{-1 / 2}(1-t)^{-1 / 2} & b t^{-1 / 2}(1-t)^{-1 / 2} \ln (1-t) \\
0 & t^{-1 / 2}(1-t)^{-1 / 2}
\end{array}\right]
$$

así deducimos que

$$
\begin{gathered}
B(P, Q)=\int_{0}^{1} t^{P-I}(1-t)^{Q-I} d t \\
=\int_{0}^{1}\left[\begin{array}{cc}
t^{-1 / 2}(1-t)^{-1 / 2} & b t^{-1 / 2}(1-t)^{-1 / 2} \ln (1-t) \\
0 & t^{-1 / 2}(1-t)^{-1 / 2}
\end{array}\right] d t=\left[\begin{array}{cc}
\pi & -2 b \pi \ln 2 \\
0 & \pi
\end{array}\right]
\end{gathered}
$$

donde según $[22$, p.558] hemos utilizado que

$$
\int_{0}^{1} \frac{t^{\mu-1} \ln (1-t)}{(1-t)^{1-\nu}} d t=B(\mu, \nu)[\psi(\nu)-\psi(\mu+\nu)], \quad \operatorname{Re}(\mu)>0, \operatorname{Re}(\nu)>0,
$$

aplicada para $\mu=1 / 2$ y $\nu=1 / 2$, en cuyo caso se deduce

$$
\int_{0}^{1} t^{-1 / 2}(1-t)^{-1 / 2} \ln (1-t) d t=B(1 / 2,1 / 2)[\psi(1 / 2)-\psi(1 / 2+1 / 2)]
$$

y ahora teniendo en cuenta que $B(1 / 2,1 / 2)=\pi$ y $\psi(1)=-C$, se deduce

$$
\int_{0}^{1} t^{-1 / 2}(1-t)^{-1 / 2} \ln (1-t) d t=-2 \pi \ln 2
$$


Con todo ello tenemos

$$
\begin{gathered}
\Gamma(P) \Gamma(Q) \Gamma^{-1}(P+Q)= \\
=\left[\begin{array}{cc}
\sqrt{\pi} & 0 \\
0 & \sqrt{\pi}
\end{array}\right]\left[\begin{array}{cc}
\sqrt{\pi} & -b \sqrt{\pi}(C+2 \ln 2) \\
0 & \sqrt{\pi}
\end{array}\right]\left[\begin{array}{cc}
1 & -b C \\
0 & 1
\end{array}\right]^{-1} \\
=\left[\begin{array}{cc}
\pi & -2 b \pi \ln 2 \\
0 & \pi
\end{array}\right]=B(P, Q)
\end{gathered}
$$

por lo que aún eliminando la hipótesis de diagonalización de ambas matrices $P$ y $Q$, la relación

$$
B(P, Q)=\Gamma(P) \Gamma(Q) \Gamma^{-1}(P+Q)
$$

sigue siendo válida.

Como hemos dicho anteriormente en lo que sigue relajaremos la condición de diagonalización de las matrices $P$ y $Q$.

Conviene señalar que implícitamente se utilizará la identidad

$$
\sigma(A+n I)=\sigma(A)+n, \quad A \in C^{r \times r}, \quad n \text { entero }
$$

Para alcanzar el objetivo anteriormente mencionado empezamos dando el siguiente

Lema 2.2.3 Sean $\hat{P}, \hat{Q} \in C^{r \times r}$ de modo que $\hat{P} \hat{Q}=\hat{Q} \hat{P}, \hat{P}+\hat{Q}+n I$ es invertible $\forall n \geq 0$ y

$$
\begin{array}{ll}
\operatorname{Re}(z)>0 & \forall \in \sigma(\hat{P}) \\
\operatorname{Re}(w)>0 & \forall \in \sigma(\hat{Q})
\end{array}
$$

entonces

$$
\begin{array}{lll}
\text { (i) } & B(\hat{P}, \hat{Q}+n I)=(\hat{P}+\hat{Q})_{n}^{-1}(\hat{Q})_{n} B(\hat{P}, \hat{Q}), & n \geq 0 \\
\text { (ii }) & B(\hat{P}+n I, \hat{Q}+n I)=(\hat{P})_{n}(\hat{Q})_{n}(\hat{P}+\hat{Q})_{2 n}^{-1} B(\hat{P}, \hat{Q}), & n \geq 0
\end{array}
$$


Demostración. Empezamos probando (i). Notar que la identidad es trivial para $\mathrm{n}=0$, ya que, por $(2.5)$ se tiene que $(M)_{0}=I, \quad M \in C^{r \times r}$. Sea ahora un natural $n \geq 1$ arbitrario, pero fijo y observemos que razonando igual que para justificar la representación integral (2.21) es sencillo probar que la integral matricial

$$
\int_{0}^{1} t^{\hat{P}-I}(1-t)^{\hat{Q}+(n-1) I} d t \quad \text { converge }
$$

por lo que podemos escribir

$$
\begin{gathered}
B(\hat{P}, \hat{Q}+n I)=\int_{0}^{1} t^{\hat{P}-I}(1-t)^{\hat{Q}+(n-1) I} d t \\
=\lim _{\delta \rightarrow 0} \int_{\delta}^{1-\delta} t^{\hat{P}-I}(1-t)^{\hat{Q}+(n-1) I} d t \\
=\lim _{\delta \rightarrow 0} \int_{\delta}^{1-\delta} t^{\hat{P}+\hat{Q}+(n-2) I}(1-t)^{\hat{Q}+(n-1) I} t^{-(\hat{Q}+(n-1) I)} d t=
\end{gathered}
$$

integrando por partes

$$
\begin{gathered}
=\lim _{\delta \rightarrow 0}\left[(\hat{P}+\hat{Q}+(n-1) I)^{-1}(1-t)^{\hat{Q}+(n-1) I} t^{\hat{P}}\right]_{\delta}^{1-\delta} \\
+\lim _{\delta \rightarrow 0}(\hat{P}+\hat{Q}+(n-1) I)^{-1} \cdot \\
\cdot \int_{\delta}^{1-\delta}\left\{(\hat{Q}+(n-1) I)(1-t)^{\hat{Q}+(n-2) I} t^{\hat{P}}+(1-t)^{\hat{Q}+(n-1) I}(\hat{Q}+(n-1) I) t^{\hat{P}-I}\right\} d t \\
=(\hat{P}+\hat{Q}+(n-1) I)^{-1}(\hat{Q}+(n-1) I) \lim _{\delta \rightarrow 0} \int_{\delta}^{1-\delta} t^{\hat{P}-I}(1-t)^{\hat{Q}+(n-2) I} d t \\
=(\hat{P}+\hat{Q}+(n-1) I)^{-1}(\hat{Q}+(n-1) I) \int_{0}^{1} t^{\hat{P}-I}(1-t)^{\hat{Q}+(n-2) I} d t \\
=(\hat{P}+\hat{Q}+(n-1) I)^{-1}(\hat{Q}+(n-1) I) B(\hat{P}, \hat{Q}+(n-1) I)
\end{gathered}
$$

donde hemos usado implícitamente en las manipulaciones la conmutatividad 
de las matrices $\hat{P}$ y $\hat{Q}$, y en la última igualdad que

$$
\int_{0}^{1} t^{\hat{P}-I}(1-t)^{\hat{Q}+(n-2) I} d t \quad \text { converge }
$$

Por tanto hemos obtenido

$$
\left.\begin{array}{c}
B(\hat{P}, \hat{Q}+n I) \\
=(\hat{P}+\hat{Q}+(n-1) I)^{-1}(\hat{Q}+(n-1) I) B(\hat{P}, \hat{Q}+(n-1) I)
\end{array}\right]
$$

Razonando como antes se obtiene igual que

$$
\left.\begin{array}{c}
B(\hat{P}, \hat{Q}+(n-1) I) \\
=(\hat{P}+\hat{Q}+(n-2) I)^{-1}(\hat{Q}+(n-2) I) B(\hat{P}, \hat{Q}+(n-2) I)
\end{array}\right]
$$

por lo que sustituyendo (2.31) en (2.30) y teniendo en cuenta que $\hat{P} \hat{Q}=\hat{Q} \hat{P}$ deducimos que

$$
\begin{gathered}
B(\hat{P}, \hat{Q}+n I) \\
=(\hat{P}+\hat{Q}+(n-1) I)^{-1}(\hat{P}+\hat{Q}+(n-2) I)^{-1} . \\
\cdot(\hat{Q}+(n-1) I)(\hat{Q}+(n-2) I) B(\hat{P}, \hat{Q}+(n-2) I)
\end{gathered}
$$

Prosiguiendo este camino de descenso se conjetura que

$$
B(\hat{P}, \hat{Q}+n I)=(\hat{P}+\hat{Q}+(n-1) I)^{-1} \ldots(\hat{P}+\hat{Q})^{-1}(\hat{Q}+(n-1) I) \ldots \hat{Q} B(\hat{P}, \hat{Q})
$$

y usando (2.5) sobre las matrices $\hat{Q}, \hat{P}+\hat{Q}$, sabemos

$$
\begin{gathered}
(\hat{P}+\hat{Q})_{n}=(\hat{P}+\hat{Q}) \ldots(\hat{P}+\hat{Q}+(n-1) I) \\
(\hat{Q})_{n}=\hat{Q} \ldots(\hat{Q}+(n-1) I)
\end{gathered}
$$


por lo que la última identidad se reescribe en términos del factorial generalizado matricial como

$$
B(\hat{P}, \hat{Q}+n I)=(\hat{P}+\hat{Q})_{n}^{-1}(\hat{Q})_{n} B(\hat{P}, \hat{Q})
$$

la cual es fácil de demostrar por inducción.

Veamos ahora la prueba de la identidad (ii), para $n \geq 1$, pues si $n=0$ la demostración es trivial.

Como

$$
\int_{0}^{1} t^{\hat{P}}(1-t)^{\hat{Q}} d t \quad \text { converge }
$$

se tiene

$$
\begin{gathered}
B(\hat{P}+I, \hat{Q}+I)=\int_{0}^{1} t^{\hat{P}}(1-t)^{\hat{Q}} d t \\
=\lim _{\delta \rightarrow 0} \int_{\delta}^{1-\delta} t^{\hat{P}}(1-t)^{\hat{Q}} d t=\lim _{\delta \rightarrow 0} \int_{\delta}^{1-\delta} t^{\hat{P}}(1-t)^{-\hat{P}}(1-t)^{\hat{P}+\hat{Q}} d t \\
=\lim _{\delta \rightarrow 0}\left[(\hat{P}+\hat{Q}+I)^{-1} t^{\hat{P}}(1-t)^{\hat{Q}+I}\right]_{\delta}^{1-\delta} \\
+\lim _{\delta \rightarrow 0} \hat{P}(\hat{P}+\hat{Q}+I)^{-1} \int_{\delta}^{1-\delta}\left[t^{\hat{P}-I}(1-t)^{-\hat{P}}+t^{\hat{P}}(1-t)^{-(\hat{P}+I)}\right](1-t)^{\hat{P}+\hat{Q}+I} d t \\
=\hat{P}(\hat{P}+\hat{Q}+I)^{-1} \lim _{\delta \rightarrow 0} \int_{\delta}^{1-\delta}\left[t^{\hat{P}-I}(1-t)^{\hat{Q}+I}+t^{\hat{P}}(1-t)^{\hat{Q}}\right] d t \\
=\hat{P}(\hat{P}+\hat{Q}+I)^{-1} \lim _{\delta \rightarrow 0} \int_{\delta}^{1-\delta} t^{\hat{P}-I}(1-t)^{\hat{Q}} d t \\
=\hat{P}(\hat{P}+\hat{Q}+I)^{-1} B(\hat{P}, \hat{Q}+I)
\end{gathered}
$$

luego hemos concluido que

$$
B(\hat{P}+I, \hat{Q}+I)=\hat{P}(\hat{P}+\hat{Q}+I)^{-1} B(\hat{P}, \hat{Q}+I)
$$

FUNC. ESPECIALES Y EC. DIF. MATRICIALES 
aplicando el apartado (i) ya demostrado, y la conmutatividad de las matrices $\hat{P}$ y $\hat{Q}$, deducimos que

$$
\begin{gathered}
B(\hat{P}+I, \hat{Q}+I)=\hat{P}(\hat{P}+\hat{Q}+I)^{-1}(\hat{P}+\hat{Q})^{-1} \hat{Q} B(\hat{P}, \hat{Q}) \\
=\hat{P} \hat{Q}(\hat{P}+\hat{Q}+I)^{-1}(\hat{P}+\hat{Q})^{-1} B(\hat{P}, \hat{Q})
\end{gathered}
$$

por lo tanto (2.29) es cierta para $n=1$.

Supongamos por hipótesis de inducción que (2.29) es válida para un cierto $n$, esto es,

$$
B(\hat{P}+n I, \hat{Q}+n I)=(\hat{P})_{n}(\hat{Q})_{n}(\hat{P}+\hat{Q})_{2 n}^{-1} B(\hat{P}, \hat{Q})
$$

y veamos que también se cumple para $n+1$, es decir

$$
B(\hat{P}+(n+1) I, \hat{Q}+(n+1) I)=(\hat{P})_{n+1}(\hat{Q})_{n+1}(\hat{P}+\hat{Q})_{2 n+2}^{-1} B(\hat{P}, \hat{Q})
$$

En efecto, usando que $\hat{P} \hat{Q}=\hat{Q} \hat{P}$,

$$
\begin{gathered}
(\hat{P})_{n+1}(\hat{Q})_{n+1}(\hat{P}+\hat{Q})_{2 n+2}^{-1} B(\hat{P}, \hat{Q}) \\
=\hat{P}(\hat{P}+I)_{n} \hat{Q}(\hat{Q}+I)_{n}(\hat{P}+\hat{Q})^{-1}(\hat{P}+\hat{Q}+I)^{-1}(\hat{P}+\hat{Q}+2 I)_{2 n}^{-1} B(\hat{P}, \hat{Q}) \\
=\hat{P} \hat{Q}(\hat{P}+\hat{Q})^{-1}(\hat{P}+\hat{Q}+I)^{-1} B(\hat{P}, \hat{Q})(\hat{P}+I)_{n}(\hat{Q}+I)_{n}(\hat{P}+\hat{Q}+2 I)_{2 n}^{-1} \\
=B(\hat{P}+I, \hat{Q}+I)(\hat{P}+I)_{n}(\hat{Q}+I)_{n}(\hat{P}+\hat{Q}+2 I)_{2 n}^{-1}=
\end{gathered}
$$

aplicando la hipótesis de inducción continuamos con la última igualdad

$$
=B(\hat{P}+(n+1) I, \hat{Q}+(n+1) I)
$$

como queríamos demostrar.

Con objeto de dar validez a la fórmula que relaciona las funciones Beta y Gamma matriciales probada en el teorema 2.2.2 anterior, pero ahora a partir de hipótesis distintas, y en un recinto más amplio, introducimos a continuación la siguiente

FUNC. ESPECIALES Y EC. DIF. MATRICIALES 
Definición 2.2.1 Dadas las matrices $P, Q \in C^{r \times r}$, de manera que $P$ y $Q$ conmutan, y $P+n I, Q+n I$ y $P+Q+n I$ son invertibles para todo entero no negativo $n$, definimos

$$
B(P, Q)=(P)_{n_{0}}^{-1}(Q)_{n_{0}}^{-1}(P+Q)_{2 n_{0}} B\left(P+n_{0} I, Q+n_{0} I\right)
$$

siendo $n_{0}=\left[\left|\tau_{0}\right|\right]+1$, donde $|\cdot|$ denota el valor absoluto, [.] la parte entera $y$ $\tau_{0}=\min \left\{\tau_{1}, \tau_{2}, \tau_{3}\right\}$ con

$$
\begin{aligned}
& \tau_{1}=\min \{\operatorname{Re}(z): z \in \sigma(P)\} \\
& \tau_{2}=\min \{\operatorname{Re}(w): w \in \sigma(Q)\} \\
& \tau_{3}=\min \{\operatorname{Re}(s): s \in \sigma(P+Q)\}
\end{aligned}
$$

Nota 2. Observamos que usando el lema 2.2.3 anterior, obtenemos que esta definición es buena, ya que, coincide con la que teníamos hasta ahora.

Con todo ello estamos ya preparados para demostrar el siguiente

Teorema 2.2.3 Sean $P, Q$ matrices en $C^{r \times r}$ verificando $P Q=Q P$ y de modo que

$$
\sigma(P), \sigma(Q), \sigma(P+Q) \subset C-\{-n, \quad n \geq 0\}
$$

entonces

$$
B(P, Q)=\Gamma(P) \Gamma(Q) \Gamma^{-1}(P+Q)
$$

Demostración. En primer lugar demostraremos el resultado para el caso particular en que

$$
\left.\begin{array}{ll}
\operatorname{Re}(z)>0 & \forall z \in \sigma(P) \\
\operatorname{Re}(w)>0 & \forall w \in \sigma(Q) \\
\operatorname{Re}(s)>0 & \forall s \in \sigma(P+Q)
\end{array}\right\}
$$

y luego abordaremos el caso general. 
Bajo la hipótesis (2.33) se tiene

$$
\begin{gathered}
\Gamma(P) \Gamma(Q)=\left(\int_{0}^{\infty} e^{-u} u^{P-I} d u\right)\left(\int_{0}^{\infty} e^{-v} v^{Q-I} d v\right) \\
=\int_{0}^{\infty} \int_{0}^{\infty}\left(e^{-u} u^{P-I} e^{-v} v^{Q-I} d u d v\right)=
\end{gathered}
$$

efectuando el cambio de variable

$$
\begin{aligned}
& x=\frac{u}{u+v} \quad \rightarrow \quad u=x y \\
& y=u+v \quad \rightarrow \quad v=y(1-x)
\end{aligned}
$$

y como el jacobiano $J(x, y)$ de esta transformación es

$$
J(x, y)=\left|\begin{array}{rc}
y & x \\
-y & 1-x
\end{array}\right|=y
$$

se tiene continuando con la última igualdad

$$
=\int_{0}^{\infty} \int_{0}^{1} e^{-x y}(x y)^{P-I} e^{-y(1-x)}[y(1-x)]^{Q-I} y d x d y=
$$

por la conmutatividad de $P$ y $Q$

$$
\begin{gathered}
=\int_{0}^{\infty} \int_{0}^{1} e^{-y} y^{P+Q-I} x^{P-I}(1-x)^{Q-I} d x d y \\
=\left(\int_{0}^{\infty} e^{-y} y^{P+Q-I} d y\right)\left(\int_{0}^{1} x^{P-I}(1-x)^{Q-I} d x\right) \\
=\Gamma(P+Q) B(P, Q)
\end{gathered}
$$

Es interesante señalar hemos utilizado la representación integral $\operatorname{de} \Gamma(P+Q)$ gracias a la hipótesis (2.33), $R e(s)>0 \quad \forall s \in \sigma(P+Q)$.

En consecuencia, hemos deducido que

$$
\Gamma(P) \Gamma(Q)=\Gamma(P+Q) B(P, Q)
$$


luego

$$
B(P, Q)=\Gamma^{-1}(P+Q) \Gamma(P) \Gamma(Q)=\Gamma(P) \Gamma(Q) \Gamma^{-1}(P+Q)
$$

tal y como queríamos demostrar.

Analizamos ahora el caso general, esto es, supongamos $P, Q$ matrices en $C^{r \times r}$ que conmutan y cumplen (2.32).

Sean

$$
\begin{array}{ll}
\tau_{1}=\min \{\operatorname{Re}(z): & z \in \sigma(P)\} \\
\tau_{2}=\min \{\operatorname{Re}(w): & w \in \sigma(Q)\} \\
\tau_{3}=\min \{\operatorname{Re}(s): & s \in \sigma(P+Q)\}
\end{array}
$$

y llamemos

$$
\tau_{0}=\min \left\{\tau_{1}, \tau_{2}, \tau_{3}\right\}
$$

entonces tomando $n_{0}=\left[\left|\tau_{0}\right|\right]+1$, siendo $[\cdot]$ y $|\cdot|$ la parte entera y el valor absoluto respectivamente, es claro que

$$
\operatorname{Re}(z)>0 \quad \forall z \in \sigma\left(P+n_{0} I\right), \sigma\left(Q+n_{0} I\right), \sigma\left(P+Q+n_{0} I\right)
$$

ya que, $\sigma(A+n I)=\sigma(A)+n, \quad A \in C^{r \times r}$.

Por otra parte, como trabajamos bajo la hipótesis (2.32) sabemos que

$$
\begin{gathered}
\Gamma(P)=\Gamma\left(P+n_{0} I\right)\left(P+\left(n_{0}-1\right) I\right)^{-1} \ldots(P+I)^{-1} P^{-1} \\
\Gamma(Q)=\Gamma\left(Q+n_{0} I\right)\left(Q+\left(n_{0}-1\right) I\right)^{-1} \ldots(Q+I)^{-1} Q^{-1} \\
\Gamma(P+Q)=\Gamma\left(P+Q+2 n_{0} I\right)\left(P+Q+\left(2 n_{0}-1\right) I\right)^{-1} \ldots(P+Q+I)^{-1}(P+Q)^{-1}
\end{gathered}
$$


en consecuencia usando $P Q=Q P$

$$
\begin{gathered}
\Gamma(P) \Gamma(Q) \Gamma^{-1}(P+Q)=\Gamma\left(P+n_{0} I\right) \Gamma\left(Q+n_{0} I\right) \Gamma^{-1}\left(P+Q+2 n_{0} I\right) \\
\cdot\left(P+\left(n_{0}-1\right) I\right)^{-1} \ldots(P+I)^{-1} P^{-1}\left(Q+\left(n_{0}-1\right) I\right) \ldots(Q+I)^{-1} Q^{-1} \\
\cdot\left(P+Q+\left(2 n_{0}-1\right) I\right) \ldots(P+Q+I)(P+Q) \\
=\Gamma\left(P+n_{0} I\right) \Gamma\left(Q+n_{0} I\right) \Gamma^{-1}\left(P+Q+2 n_{0} I\right)(P)_{n_{0}}^{-1}(Q)_{n_{0}}^{-1}(P+Q)_{2 n_{0}} \\
=B\left(P+n_{0} I, Q+n_{0} I\right)(P)_{n_{0}}^{-1}(Q)_{n_{0}}^{-1}(P+Q)_{2 n_{0}}=B(P, Q)
\end{gathered}
$$

por lo tanto hemos deducido

$$
B(P, Q)=\Gamma(P) \Gamma(Q) \Gamma^{-1}(P+Q)
$$

siendo $P$ y $Q$ matrices que conmutan y cumplen (2.32), con lo que el resultado queda probado. 


\section{Capítulo 3}

\section{SOBRE LA FUNCION Y LA ECUACION DIFERENCIAL HIPERGEOMETRICA MATRICIAL}

\subsection{Introducción}

Empezamos este tercer capítulo recordando en primer lugar, que las referencias bibliográficas [45], [47], [49], [52] se dedican al estudio de las funciones especiales, de la física matemática y de los polinomios ortogonales. Asímismo, en los trabajos [10], [28], [49] y [16], [32], [36], [33] se muestran las funciones especiales matriciales en conexión con la Estadística, la Física Teórica, la teoría de representación de grupos y los polinomios ortogonales matriciales.

El propósito de este capítulo es el estudio de la ecuación diferencial hipergeométrica matricial y su conexión con la función hipergeométrica matricial. La función hipergeométrica matricial es interesante para desarrollar la relación entre la emergente teoría de los polinomios matriciales ortogonales y las ecuaciones diferenciales matriciales, así como la evaluación exacta de ciertas integrales que involucran funciones matriciales en términos de las funciones matriciales hipergeométricas. 
Este capítulo está organizado de la siguiente forma. En el segundo apartado resumimos algunos resultados sobre ecuaciones diferenciales matriciales bilaterales e introducimos el concepto de conjunto fundamental de soluciones para una ecuación diferencial matricial del tipo

$$
X^{\prime \prime}=f_{1}(z) X^{\prime}+f_{2}(z) X f_{3}(z)+X^{\prime} f_{4}(z),
$$

donde $f_{i}(z)$ son funciones con valores matriciales de variable compleja $z$.

En el tercer apartado se introduce la función hipergeométrica matricial $F(A, B ; C ; z)$. Se dan algunas de las propiedades de $F(A, B ; C ; z)$, tales como su invertibilidad, cotas de error para su desarrollo en serie truncado, así como cotas para su inversa y para su derivada en términos de los datos.

En el apartado cuarto se aporta la solución general en forma cerrada de la ecuación diferencial hipergeométrica matricial

$$
z(1-z) W^{\prime \prime}-z A W^{\prime}+W^{\prime}(C-z(B+I))-A W B=0,
$$

donde $A, B, C$ son matrices en $C^{r \times r}$ y $z$ es un número complejo.

En el apartado quinto, aprovechando la representación límite de la función Gamma matricial dada en el segundo capítulo, se demuestra una condición suficiente para garantizar la convergencia de $F(A, B ; C ; z)$ en la frontera de su dominio de convergencia.

En la sexta y última sección, bajo ciertas hipótesis se da una representación integral de $F(A, B ; C ; z)$. Para ello utilizaremos la relación entre las funciones Beta y Gamma matriciales establecida en el capítulo anterior.

Si $P$ es una matriz en $C^{p \times q}$, su 2-norma, denotada por $\|P\|$, está definida por $[21$, p.56]

$$
\|P\|=\sup _{x \neq 0} \frac{\|P x\|_{2}}{\|x\|_{2}}
$$

donde para un vector $y$ de $C^{q},\|y\|_{2}=\left(y^{T} y\right)^{1 / 2}$ es la norma euclídea usual de $y$.

FUNC. ESPECIALES Y EC. DIF. MATRICIALES 
Recordemos, pues más adelante nos será útil, que por el lema de perturbación [15, p.5], si $P, Q$ son matrices en $C^{r \times r}$ siendo $Q$ invertible, y si

$$
\|P-Q\|<\left\|Q^{-1}\right\|^{-1},
$$

entonces $P$ es invertible y

$$
\begin{gathered}
\left\|P^{-1}\right\| \leq \frac{\left\|Q^{-1}\right\|}{1-\left\|Q^{-1}\right\|\|P-Q\|} \\
\left\|P^{-1}-Q^{-1}\right\| \leq \frac{\left\|Q^{-1}\right\|^{2}\|P-Q\|}{1-\left\|Q^{-1}\right\|\|P-Q\|} .
\end{gathered}
$$

\subsection{Ecuaciones diferenciales matriciales linea- les de segundo orden bilaterales}

Para mayor claridad en el desarrollo de este segundo apartado, recordamos previamente un resultado cuya demostración puede encontrarse en el texto [14, capítulo X].

Teorema 3.2.1 ([14, p.287]). Sea B $\left(z_{0} ; r\right)$ un disco abierto en el plano complejo de radio $r$ centrado en el punto $z_{0}$. Sea $E$ el espacio de Banach de todas las matrices de $C^{r \times r}$ dotado con la 2-norma. Sea $f: B\left(z_{0} ; r\right) \times E \rightarrow E$ una función continua, tal que

$$
\left\|f\left(z, X_{1}\right)-f\left(z, X_{2}\right)\right\| \leq K\left(\left|z-z_{0}\right|\right)\left\|X_{1}-X_{2}\right\|,
$$

donde $z \in B\left(z_{0} ; r\right) ; X_{i}$ varía en $E$ para $i=1,2$ y $\xi \rightarrow K(\xi)$ es una función continua a valores reales definida sobre el intervalo $[0, r]$. Entonces para cada $X_{0}$ en $E$, existe una y sólo una solución $U$ de

$$
X^{\prime}=f(z, X),
$$

definida en $B\left(z_{0} ; r\right)$, tal que $U\left(z_{0}\right)=X_{0}$.

FUNC. ESPECIALES Y EC. DIF. MATRICIALES 
En particular, si consideramos una ecuación diferencial matricial lineal bilateral de la forma

$$
X^{\prime}=A_{1}(z) X+X B_{1}(z)+A_{2}(z) X B_{2}(z)+A_{3}(z) X B_{3}(z)+C(z),
$$

donde $A_{i}, B_{i}$ para $i=1,2,3$, y $C$ son funciones continuas de $B\left(z_{0} ; r\right)$ en $E$, entonces la ecuación (3.6) es del tipo (3.5), donde

$$
f(z, X)=A_{1}(z) X+X B_{1}(z)+A_{2}(z) X B_{2}(z)+A_{3}(z) X B_{3}(z)+C(z),
$$

y $f$ satisface una condición de Lipschitz del tipo (3.4). Por el teorema 3.2.1 anterior, para cada $X_{0}$ en $E$, existe única solución $U$ de (3.6), para $z$ en $B\left(z_{0} ; r\right)$ de manera que $U\left(z_{0}\right)=X_{0}$.

La siguiente definición es una extensión del concepto de conjunto fundamental de soluciones introducido en [29] para un caso particular.

Definición 3.2.1. Sean $f_{j}: B\left(z_{0} ; r\right) \rightarrow$ E funciones continuas y acotadas en $B\left(z_{0} ; r\right)$ para $1 \leq j \leq 4$, y sean $U_{1}, U_{2}$ dos soluciones de la ecuación diferencial de segundo orden

$$
X^{\prime \prime}=f_{1}(z) X^{\prime}+f_{2}(z) X f_{3}(z)+X^{\prime} f_{4}(z) .
$$

Decimos que $\left\{U_{1}, U_{2}\right\}$ es un conjunto fundamental de soluciones de (3.7) en $B\left(z_{0} ; r\right)$, si cualquier solución $U$ de (3.7) admite una única representación de la forma

$$
U(z)=U_{1}(z) P+U_{2}(z) Q, \quad z \in B\left(z_{0} ; r\right),
$$

donde $P, Q$ son matrices en $C^{r \times r}$, únicamente determinadas por $U$.

Teorema 3.2.2 . Si $\left\{U_{1}, U_{2}\right\}$ es una pareja de soluciones de la ecuación (3.7) en $B\left(z_{0} ; r\right)$ de manera que la $C^{2 r \times 2 r}$ matriz

$$
W\left(U_{1}, U_{2}, z_{0}\right)=\left[\begin{array}{cc}
U_{1}\left(z_{0}\right) & U_{2}\left(z_{0}\right) \\
U_{1}^{\prime}\left(z_{0}\right) & U_{2}^{\prime}\left(z_{0}\right)
\end{array}\right] \quad \text { es invertible, }
$$

entonces $\left\{U_{1}, U_{2}\right\}$ es un conjunto fundamental de soluciones de (3.7) en $B\left(z_{0} ; r\right)$. 
Demostración. Observemos que para cualquier pareja de matrices $P, Q$ en $C^{r \times r}$, la función $V(z)=U_{1}(z) P+U_{2}(z) Q$ es una solución de (3.7). Dada una solución $U$ de (3.7) con condiciones iniciales $C_{0}=U\left(z_{0}\right), C_{1}=U^{\prime}\left(z_{0}\right)$, sean $P_{0}, Q_{0}$ las matrices definidas por

$$
\left[\begin{array}{l}
P_{0} \\
Q_{0}
\end{array}\right]=\left[W\left(U_{1}, U_{2}, z_{0}\right)\right]^{-1}\left[\begin{array}{l}
C_{0} \\
C_{1}
\end{array}\right] .
$$

Entonces $V(z)=U_{1}(z) P_{0}+U_{2}(z) Q_{0}$ es una solución de (3.7) en $B\left(z_{0} ; r\right)$, satisfaciendo las mismas condiciones iniciales que $U$ ya que,

$$
\begin{gathered}
V^{\prime}(z)=U_{1}^{\prime}(z) P_{0}+U_{2}^{\prime}(z) Q_{0}, \\
{\left[\begin{array}{c}
V\left(z_{0}\right) \\
V^{\prime}\left(z_{0}\right)
\end{array}\right]=\left[\begin{array}{cc}
U_{1}\left(z_{0}\right) & U_{2}\left(z_{0}\right) \\
U_{1}^{\prime}\left(z_{0}\right) & U_{2}^{\prime}\left(z_{0}\right)
\end{array}\right]\left[\begin{array}{c}
P_{0} \\
Q_{0}
\end{array}\right]} \\
=W\left(U_{1}, U_{2}, z_{0}\right)\left[W\left(U_{1}, U_{2}, z_{0}\right)\right]^{-1}\left[\begin{array}{c}
C_{0} \\
C_{1}
\end{array}\right] . \\
{\left[\begin{array}{c}
V\left(z_{0}\right) \\
V^{\prime}\left(z_{0}\right)
\end{array}\right]=\left[\begin{array}{c}
C_{0} \\
C_{1}
\end{array}\right]=\left[\begin{array}{c}
U\left(z_{0}\right) \\
U^{\prime}\left(z_{0}\right)
\end{array}\right] .}
\end{gathered}
$$

Ahora probamos que un problema de valor inicial para la ecuación (3.7) admite una única solución. En efecto, notemos que considerando el cambio de variable $Y=\left[\begin{array}{ll}X & X^{\prime}\end{array}\right]^{T}$, la ecuación (3.7) es equivalente a la ecuación diferencial matricial de primer orden

$$
Y^{\prime}=\left[\begin{array}{ll}
0 & I \\
0 & 0
\end{array}\right] Y+\left[\begin{array}{cc}
0 & 0 \\
0 & f_{1}(z)
\end{array}\right] Y+\left[\begin{array}{ll}
0 & 0 \\
0 & I
\end{array}\right] Y f_{4}(z)+\left[\begin{array}{cc}
0 & 0 \\
f_{2}(z) & 0
\end{array}\right] Y f_{3}(z) .
$$

Por el teorema 3.2.1 anterior, un problema de valor inicial del tipo (3.12) admite una única solución. Como la relación entre las soluciones de (3.7) y (3.12) está dada por

$$
X=\left[\begin{array}{ll}
I & 0
\end{array}\right] Y,
$$

entonces un problema de valor inicial del tipo (3.7) admite una única solución. Por (3.11) $V(z)=U_{1}(z) P_{0}+U_{2}(z) Q_{0}$ satisface la misma condición inicial que $U(z)$. Por la unicidad $V(z)=U(z)$ para todo $z$ en $B\left(z_{0} ; r\right)$. Por tanto el resultado queda demostrado.

Ejemplo 1. Sea $z_{0}$ un número complejo tal que $0<\left|z_{0}\right|<1$ y sean $A$, 
$B, C$ matrices complejas en $C^{r \times r}$. Sea $0<\delta_{0}<\left|z_{0}\right|<1-\delta_{1}$, con $0<\delta_{1}<1$ y $r=\operatorname{mín}\left(\delta_{0}, \delta_{1}\right)$. Entonces en $B\left(z_{0} ; r\right)$ la ecuación

$$
W^{\prime \prime}=\frac{A}{1-z} W^{\prime}+\frac{A}{z(1-z)} W B-W^{\prime}\left[\frac{C-z(B+I)}{z(1-z)}\right],
$$

es del tipo (3.7) con:

$$
f_{1}(z)=\frac{A}{1-z} ; \quad f_{2}(z)=\frac{A}{z(1-z)} ; \quad f_{3}(z)=B ; \quad f_{4}(z)=\frac{-C+z(B+I)}{z(1-z)} .
$$

\subsection{La función hipergeométrica matricial}

Empezamos este apartado, con la definición de la función hipergeométrica matricial.

Definición 3.3.1 . Sean $A, B, C$ matrices en $C^{r \times r}$ donde

$$
C+n I \text { es invertible para todo entero } n \geq 0 \text {, }
$$

entonces denotamos por $F(A, B ; C ; z)$ la función hipergeometrica matricial, definida por

$$
F(A, B ; C ; z)=\sum_{n \geq 0} \frac{1}{n !}(A)_{n}(B)_{n}\left[(C)_{n}\right]^{-1} z^{n} .
$$

donde la notación $(\cdot)$, está definida en (2.5) del capítulo 2.

A continuación, probaremos que la serie de potencias matricial (3.17) converge para todo $z$ tal que $|z|<1$. Notar que si $n$ es un entero positivo suficientemente grande, de modo que, $n>\|C\|$, entonces por el lema de perturbación, ver (3.2), podemos escribir

$$
\left\|\left(\frac{C}{n}+I\right)^{-1}\right\| \leq \frac{1}{1-\frac{\|C\|}{n}}=\frac{n}{n-\|C\|},
$$

FUNC. ESPECIALES Y EC. DIF. MATRICIALES 
y por tanto

$$
\left\|(C+n I)^{-1}\right\|=\left\|\frac{1}{n}\left(\frac{C}{n}+I\right)^{-1}\right\|=\frac{1}{n}\left\|\left(\frac{C}{n}+I\right)^{-1}\right\| \leq \frac{1}{n-\|C\|}, \quad n>\|C\| .
$$

En lo que sigue denotaremos

$$
\Upsilon(n)=\left\|C^{-1}\right\|\left\|(C+I)^{-1}\right\| \ldots\left\|(C+(n-1) I)^{-1}\right\|, \quad n \geq 0,
$$

y es conveniente observar, para las próximas acotaciones que

$$
\left\|(A)_{n}\right\| \leq(\|A\|)_{n}, \quad\left\|(B)_{n}\right\| \leq(\|B\|)_{n}
$$

Por (3.19), (3.20), se tiene

$$
\left\|\frac{(A)_{n}(B)_{n}\left[(C)_{n}\right]^{-1}}{n !} z^{n}\right\| \leq\left.\frac{\left\|(A)_{n}\right\|\left\|(B)_{n}\right\| \Upsilon(n)}{n !}\left|z^{n} \leq \frac{(\|A\|)_{n}(\|B\|)_{n} \Upsilon(n)}{n !}\right|\right|^{n}
$$

Ahora probamos que

$$
\sum_{n \geq 0} \frac{(\|A\|)_{n}(\|B\|)_{n} \Upsilon(n)}{n !} z^{n}
$$

converge para $|z|<1$ usando el criterio del cociente. En efecto por (3.18) se sigue que para $n>\|C\|$

$$
\begin{aligned}
& \lim _{n \rightarrow \infty} \frac{(\|A\|)_{n+1}(\|B\|)_{n+1} \Upsilon(n+1)|z|^{n+1}}{(\|A\|)_{n}(\|B\|)_{n} \Upsilon(n)|z|^{n}(n+1)} \\
= & \lim _{n \rightarrow \infty} \frac{(\|A\|+n)(\|B\|+n)\left\|(C+n I)^{-1}\right\|}{n+1}|z| \\
\leq & \lim _{n \rightarrow \infty} \frac{(\|A\|+n)(\|B\|+n)}{(n+1)(n-\|C\|)}|z|=|z| .
\end{aligned}
$$

Luego la serie de potencias (3.17) es absolutamente convergente para $|z|<1$.

Ahora estamos interesados en la determinación de un dominio en el que $U_{1}(z)=F(A, B ; C ; z)$ sea invertible. Sea $0<\gamma<1$ y notemos que

$$
\lim _{n \rightarrow \infty} \frac{(\|A\|+n)(\|B\|+n)}{(n+1)(n-\|C\|)}=1
$$

FUNC. ESPECIALES Y EC. DIF. MATRICIALES 
Tomemos $n_{0} \geq \operatorname{máx}(1,\|C\|)$ un entero positivo de manera que verifique la siguiente desigualdad

$$
\frac{(\|A\|+n)(\|B\|+n)}{(n+1)(n-\|C\|)}<1+\gamma, \quad n \geq n_{0},
$$

Consideremos ahora los números reales positivos $\Lambda$ y $L$, definidos respectivamente como

$$
\Lambda=\sum_{n=1}^{n_{0}-1} \frac{(\|A\|)_{n}(\|B\|)_{n} \Upsilon(n)}{n !} ; \quad L=\frac{(\|A\|)_{n_{0}}(\|B\|)_{n_{0}} \Upsilon\left(n_{0}\right)}{n_{0} !}
$$

donde $\Upsilon(n)$ está definido por (3.19). Observemos que como la función real de variable real

$$
f(x)=\Lambda x+L \frac{x}{1-x}, \quad 0 \leq x<1,
$$

es creciente,

$$
f(0)=0 \quad y \quad \lim _{x \rightarrow 1^{-}} f(x)=+\infty,
$$

existe un único valor $\rho_{0}$ tal que $0<\rho_{0}<1$, de forma que

$$
f\left(\rho_{0}\right)=1-\gamma .
$$

Tomamos $\rho_{1}$ con $0<\rho_{1}<\rho_{0}$, de manera que $(1+\gamma) \rho_{1}<\rho_{0}$. Por $(3.22)$ podemos escribir para $n \geq n_{0}$,

$$
\frac{\frac{1}{(n+1) !}(\|A\|)_{n+1}(\|B\|)_{n+1} \Upsilon(n+1)}{\frac{1}{n !}(\|A\|)_{n}(\|B\|)_{n} \Upsilon(n)} \leq \frac{(\|A\|+n)(\|B\|+n)}{(n+1)(n-\|C\|)} \leq 1+\gamma,
$$

y en consecuencia por la elección de $\rho_{1}$, de las desigualdades anteriores se deduce

$$
\frac{1}{(n+1) !}(\|A\|)_{n+1}(\|B\|)_{n+1} \Upsilon(n+1) \rho_{1} \leq \rho_{0} \frac{1}{n !}(\|A\|)_{n}(\|B\|)_{n} \Upsilon(n), \quad n \geq n_{0} .
$$

Luego podemos deducir la siguiente acotación

FUNC. ESPECIALES Y EC. DIF. MATRICIALES 


$$
\begin{gathered}
\left\|U_{1}\left(\rho_{1}\right)-U_{1}(0)\right\|=\left\|U_{1}\left(\rho_{1}\right)-I\right\| \\
\leq \sum_{n \geq 1} \frac{1}{n !}(\|A\|)_{n}(\|B\|)_{n} \Upsilon(n) \rho_{1}^{n} \\
\leq\left\{\sum_{n=1}^{n_{0}-1} \frac{1}{n !}(\|A\|)_{n}(\|B\|)_{n} \Upsilon(n)\right\} \rho_{1} \\
+\sum_{n \geq n_{0}} \frac{1}{n !}(\|A\|)_{n}(\|B\|)_{n} \Upsilon(n) \rho_{1}^{n} \\
\leq \Lambda \rho_{0}+\sum_{n \geq n_{0}} \frac{1}{n !}(\|A\|)_{n}(\|B\|)_{n} \Upsilon(n) \rho_{1}^{n} .
\end{gathered}
$$

donde la última serie cumple

$$
\begin{gathered}
\sum_{n \geq n_{0}} \frac{1}{n !}(\|A\|)_{n}(\|B\|)_{n} \Upsilon(n) \rho_{1}^{n} \\
\leq\left[\frac{1}{n_{0} !}(\|A\|)_{n_{0}}(\|B\|)_{n_{0}} \Upsilon\left(n_{0}\right)\right] \sum_{n \geq n_{0}} \rho_{0}^{n} \\
=\frac{1}{n_{0} !}(\|A\|)_{n_{0}}(\|B\|)_{n_{0}} \Upsilon\left(n_{0}\right) \frac{\rho_{0}^{n_{0}}}{1-\rho_{0}}<L \frac{\rho_{0}}{1-\rho_{0}} .
\end{gathered}
$$

y por (3.27), (3.28) se deduce

$$
\left\|U_{1}\left(\rho_{1}\right)-I\right\| \leq \Lambda \rho_{0}+L \frac{\rho_{0}}{1-\rho_{0}}=f\left(\rho_{0}\right)=1-\gamma .
$$

En consecuencia $\left\|U_{1}(\rho)-I\right\|<1-\gamma$, para $0 \leq \rho \leq \rho_{1}$ y por el lema de perturbación, se tiene que

$$
U_{1}(z)=F(A, B ; C ; z) \text { es invertible y }\left\|U_{1}(z)-I\right\|<1-\gamma \text { para }|z| \leq \rho_{1} \text {. (3.29) }
$$

Por (3.2) y (3.29),

$$
\left\|\left(U_{1}(z)\right)^{-1}-I\right\| \leq \frac{\left\|U_{1}(z)-I\right\|}{1-\left\|U_{1}(z)-I\right\|}<\frac{1-\gamma}{\gamma}, \quad|z| \leq \rho_{1} .
$$


$\mathrm{y}$

$$
\left\|\left(U_{1}(z)\right)^{-1}\right\| \leq 1+\frac{1-\gamma}{\gamma}=\frac{1}{\gamma}, \quad|z| \leq \rho_{1} .
$$

Además, por (3.28) para $n \geq n_{0}$ y $|z| \leq \rho_{1}$ se deduce que

$$
\begin{gathered}
\left\|U_{1}(z)-\sum_{j=1}^{n-1} \frac{1}{j !}(A)_{j}(B)_{j}\left[(C)_{j}\right]^{-1} z^{j}\right\| \\
\leq \sum_{m \geq n} \frac{1}{m !}(\|A\|)_{m}(\|B\|)_{m} \Upsilon(m) \rho_{1}^{m} \\
\leq L \sum_{m \geq n} \rho_{0}^{m}=L \frac{\rho_{0}^{n}}{1-\rho_{0}} .
\end{gathered}
$$

Luego, dado $\varepsilon>0$, tomando $n \geq n_{0}$ de modo que

$$
\rho_{0}^{n}<\frac{\varepsilon\left(1-\rho_{0}\right)}{L}
$$

o equivalentemente

$$
n \geq \operatorname{máx}\left\{\frac{\ln \left[\frac{\varepsilon\left(1-\rho_{0}\right)}{L}\right]}{\ln \left(\rho_{0}\right)}, \quad n_{0}\right\}=n_{\varepsilon},
$$

si denotamos por

$$
F_{n}(A, B ; C ; z)=\sum_{j=0}^{n} \frac{(A)_{j}(B)_{j}\left[(C)_{j}\right]^{-1} z^{j}}{j !}
$$

el truncamiento de orden $n$ de la función matricial $F(A, B ; C ; z)$, se deduce que cumple

$$
\left\|U_{1}(z)-F_{n-1}(A, B ; C ; z)\right\| \leq \varepsilon, \quad|z| \leq \rho_{1}, \quad n \geq n_{\varepsilon} .
$$

Así pues, y resumiendo los razonamientos anteriores, el siguiente resultado queda demostrado:

FUNC. ESPECIALES Y EC. DIF. MATRICIALES 
Teorema 3.3.1. Sean $0<\gamma<1$ y $\varepsilon>0$. Sean $n_{0}$ y $n_{\varepsilon}$ enteros positivos satisfaciendo (3.22) y (3.31) respectivamente. Sea $\rho_{0}$ la solución de la ecuación (3.25) donde $f$ está definida por (3.24). Sea $\rho_{1}$ de manera que $\rho_{1}<(1+\gamma)^{-1} \rho_{0}, U_{1}(z)=F(A, B ; C ; z)$ y $F_{n}(A, B ; C ; z)$ definida por (3.32). Entonces

(i) $U_{1}(z)$ es invertible $y\left\|\left(U_{1}(z)\right)^{-1}\right\| \leq \gamma^{-1}$ para $|z| \leq \rho_{1}$.

(ii) $\left\|U_{1}(z)-F_{n}(A, B ; C ; z)\right\|<\varepsilon, \quad|z| \leq \rho_{1}, \quad n \geq n_{\varepsilon}$.

Consideremos ahora las cotas para la función matricial $U_{1}^{\prime}(z)$ en el recinto $|z| \leq \rho_{1}$ donde $\rho_{1}$ está dado por el teorema 3.3.1 anterior.

Notemos que para $|z| \leq \rho_{1}$ se tiene

$$
\left\|U_{1}^{\prime}(z)\right\| \leq \sum_{n \geq 1} \frac{(\|A\|)_{n}(\|B\|)_{n} \Upsilon(n)}{(n-1) !} \rho_{1}^{n-1} .
$$

Dada $\gamma$ con $0<\gamma<1$, sea $n_{1} \geq n_{0}$ tal que

$$
\frac{(\|A\|+n)(\|B\|+n)}{n(n-\|C\|)}<1+\gamma, \quad n \geq n_{1}>\operatorname{máx}(1,\|C\|),
$$

Sea $\rho_{2}$ tal que $(1+\gamma) \rho_{2}<\rho_{1} \mathrm{y}$

$$
\Lambda_{1}=\sum_{n=1}^{n_{1}-1} \frac{(\|A\|)_{n}(\|B\|)_{n} \Upsilon(n)}{(n-1) !}, \quad L_{1}=\frac{(\|A\|)_{n_{1}}(\|B\|)_{n_{1}} \Upsilon\left(n_{1}\right)}{\left(n_{1}-1\right) !}
$$

donde $\Upsilon(n)$ está definida por (3.19). Observemos que para $|z| \leq \rho_{2}$ podemos escribir

$$
\left\|U_{1}^{\prime}(z)\right\| \leq \sum_{n=1}^{n_{1}-1} \frac{(\|A\|)_{n}(\|B\|)_{n} \Upsilon(n)}{(n-1) !} \rho_{2}^{n-1}+\sum_{n \geq n_{1}} \frac{(\|A\|)_{n}(\|B\|)_{n} \Upsilon(n)}{(n-1) !} \rho_{2}^{n-1},
$$

y por (3.33)

$$
\frac{1}{n !}(\|A\|)_{n+1}(\|B\|)_{n+1} \Upsilon(n+1)<(1+\gamma) \frac{1}{(n-1) !}(\|A\|)_{n}(\|B\|)_{n} \Upsilon(n), \quad n \geq n_{1},
$$

FUNC. ESPECIALES Y EC. DIF. MATRICIALES 
se deduce

$$
\left.\begin{array}{c}
\sum_{n \geq n_{1}} \frac{(\|A\|)_{n}(\|B\|)_{n} \Upsilon(n)}{(n-1) !} \rho_{2}^{n-1} \\
\leq \frac{(\|A\|)_{n_{1}}(\|B\|)_{n_{1}} \Upsilon\left(n_{1}\right)}{\left(n_{1}-1\right) !} \sum_{n \geq n_{1}}\left[\rho_{2}(1+\gamma)\right]^{n-1} \\
\leq L_{1} \sum_{n \geq n_{1}} \rho_{1}^{n-1}=\frac{L_{1} \rho_{1}^{n_{1}-1}}{1-\rho_{1}} \leq \frac{L_{1}}{1-\rho_{1}}
\end{array}\right]
$$

Si $F_{n}(A, B ; C ; z)$ está definida por (3.32), usando (3.34)-(3.36) se tienen las siguientes acotaciones

$$
\begin{gathered}
\left\|U_{1}^{\prime}(z)-F_{n}^{\prime}(A, B ; C ; z)\right\| \leq \frac{L_{1}}{1-\rho_{1}} \rho_{1}^{n_{1}-1}, \quad n \geq n_{1}, \quad|z| \leq \rho_{2}, \\
\left\|U_{1}^{\prime}(z)\right\| \leq \Lambda_{1}+\frac{L_{1}}{1-\rho_{1}}, \quad|z| \leq \rho_{2} .
\end{gathered}
$$

Resumiendo, el siguiente resultado que nos proporciona cotas para $U_{1}^{\prime}(z)$ y para su truncamiento de orden $n$, queda probado:

Corolario 3.3.1 . Con la notación del teorema 3.3.1 anterior, sea $\rho_{2}>0$ elegido de manera que $\rho_{2}(1+\gamma)<\rho_{1}$. Sea $n_{1}$ un entero positivo satisfaciendo (3.33) $y$

$$
n_{\varepsilon}^{\prime}=1+\operatorname{máx}\left\{\frac{\ln \left(\frac{\varepsilon\left(1-\rho_{1}\right)}{L_{1}}\right)}{\ln \left(\rho_{1}\right)}, \quad n_{1}\right\},
$$

donde $\Lambda_{1}$ y $L_{1}$ están definidas por (3.34). Entonces

(i) $\left\|U_{1}^{\prime}(z)\right\| \leq \Lambda_{1}+\left(1-\rho_{1}\right)^{-1} L_{1}, \quad|z| \leq \rho_{2}$.

(ii) $\left\|U_{1}^{\prime}(z)-F_{n}^{\prime}(A, B ; C ; z)\right\| \leq \varepsilon, \quad|z| \leq \rho_{2}, \quad n \geq n_{\varepsilon}^{\prime}$

Los resultados establecidos en este apartado los utilizaremos próximamente para determinar la solución general de la ecuación diferencial hipergeométrica matricial.

FUNC. ESPECIALES Y EC. DIF. MATRICIALES 


\subsection{La ecuación diferencial hipergeométrica ma- tricial}

Consideremos la ecuación diferencial matricial hipergeométrica

$$
z(1-z) W^{\prime \prime}-z A W^{\prime}+W^{\prime}(C-z(B+I))-A W B=0, \quad 0<|z|<1,
$$

donde

$$
C B=B C .
$$

y $C$ satiface (3.16). Notar que la ecuación (3.39) puede escribirse en la forma (3.14). Buscamos una solución $W$ de (3.39) de la forma

$$
W(z)=\sum_{n \geq 0} W_{n} z^{n}, \quad|z|<1 .
$$

Tomando derivadas formales en (3.41) y sustituyendo en (3.39) se tiene

$$
\begin{gathered}
W^{\prime}(z)=\sum_{n \geq 1} n W_{n} z^{n-1}, \quad W^{\prime \prime}(z)=\sum_{n \geq 2} n(n-1) W_{n} z^{n-2}, \\
\sum_{n \geq 2} n(n-1) W_{n} z^{n-1}-\sum_{n \geq 2} n(n-1) W_{n} z^{n}-\sum_{n \geq 1} n A W_{n} z^{n}-\sum_{n \geq 1} n W_{n} B z^{n} \\
+\sum_{n \geq 1} n W_{n} C z^{n-1}-\sum_{n \geq 1} n W_{n} z^{n}-\sum_{n \geq 0} A W_{n} B z^{n}=0, \\
\left(W_{1} C-A W_{0} B\right)+\left(2 W_{2}-A W_{1}-W_{1} B-W_{1}+2 W_{2} C-A W_{1} B\right) z \\
+\sum_{n \geq 2}\left\{n(n+1) W_{n+1}-n(n-1) W_{n}-n A W_{n}-n W_{n} B-n W_{n}+(n+1) W_{n+1} C-A W_{n} B\right\} z^{n}=0
\end{gathered}
$$

Igualando a cero los coeficientes de cada potencia de $z^{n}$ se tiene que

$$
\left.\begin{array}{c}
W_{1} C-A W_{0} B=0, \\
2 W_{2}-A W_{1}-W_{1} B-W_{1}+2 W_{2} C-A W_{1} B=0, \\
n(n+1) W_{n+1}-n(n-1) W_{n}-n A W_{n} \\
-n W_{n} B-n W_{n}+(n+1) W_{n+1} C-A W_{n} B=0
\end{array}\right]
$$

FUNC. ESPECIALES Y EC. DIF. MATRICIALES 
Luego

$$
\begin{gathered}
W_{1}=A W_{0} B C^{-1}, \\
W_{n+1}=\frac{1}{(n+1)}(A+n I) W_{n}(B+n I)(n I+C)^{-1}, \quad n \geq 0
\end{gathered}
$$

Observar que por (3.44) y (3.40), tomando $W_{0}=I$ se deduce que

$$
\begin{gathered}
W_{n+1}=\frac{1}{(n+1) !} A(A+I) \ldots(A+n I) B(B+I) \ldots(B+n I)(C+n I)^{-1} \ldots(C+I)^{-1} C^{-1} \\
W_{n+1}=\frac{1}{(n+1) !}(A)_{n+1}(B)_{n+1}\left[(C)_{n+1}\right]^{-1} .
\end{gathered}
$$

Luego existe una solución $W_{1}$ de (3.39) la cual está bien definida en $|z|<1$ satisfaciendo $W_{1}(0)=I$, dada por

$$
W_{1}(z)=F(A, B ; C ; z) .
$$

Ahora buscaremos una segunda solución $W_{2}$ de la ecuación (3.39), bajo las hipótesis: $C+n I$ invertible para todo entero $n \leq-2$, (3.40) junto con

$$
A C=C A .
$$

Sea $D_{0}$ el plano complejo cortado a lo largo del eje real negativo, y denotemos por $z^{I-C}=\exp ((I-C) \log z)$ donde log representa la determinación principal del logaritmo, [56, p.72]. Buscamos una solución de la forma

$$
W_{2}(z)=V(z) z^{I-C}, \quad|z|<1, \quad z \in D_{0},
$$

donde $V$ es una función por determinar. Tomando derivadas de $W_{2}$ se tiene

$$
\begin{gathered}
W_{2}^{\prime}(z)=V^{\prime}(z) z^{I-C}+V(z) z^{-C}(I-C), \\
W_{2}^{\prime \prime}(z)=V^{\prime \prime}(z) z^{I-C}+2 V^{\prime}(z) z^{-C}(I-C)-V(z) z^{-C-I} C(I-C) .
\end{gathered}
$$

Sustituyendo estas expresiones en (3.39), y denotando $V(z)=V$, se deduce

$$
\left.\begin{array}{c}
z(1-z) V^{\prime \prime}+\left\{2 V^{\prime}-V^{\prime} C-3 z V^{\prime}+2 z V^{\prime} C-A z V^{\prime}-z V^{\prime} B\right\} \\
+\left\{V C-V C^{2}-A V+A V C-V B-V+V C B+V C-A V B\right\}=0
\end{array}\right]
$$


Supongamos por un momento que

$$
V(z) C=C V(z), \quad V^{\prime}(z) C=C V^{\prime}(z)
$$

entonces (3.49) es equivalente a

$$
z(1-z) V^{\prime \prime} z(A+I-C) V^{\prime}+V^{\prime}[(2 I-C)-z(B+2 I-C)]-(A+I-C) V(B+I-C)=0 .
$$

Observar que la ecuación (3.51) es una ecuación diferencial hipergeométrica matricial del tipo (3.39) con parámetros

$$
A^{\prime}=A+I-C, \quad B^{\prime}=B+I-C, \quad C^{\prime}=2 I-C .
$$

Como probamos anteriormente

$$
V(z)=F(A+I-C, B+I-C ; 2 I-C ; z), \quad|z|<1,
$$

es una solución de (3.51) porque $C B=B C$. Además, notemos que bajo las hipótesis (3.40) y (3.47), efectivamente $F(A+I-C, B+I-C ; 2 I-C ; z)$ y su derivada conmutan con $C$ pues los coeficientes matriciales de la función $F(A+I-C, B+I-C ; 2 I-C ; z)$ son

$$
\frac{1}{n !}(A+I-C)_{n}(B+I-C)_{n}\left[(2 I-C)_{n}\right]^{-1}, \quad n \geq 0 .
$$

Por tanto

$$
W_{2}(z)=F(A+I-C, B+I-C ; 2 I-C ; z) z^{I-C},
$$

es además una solución de (3.39) para $z \in D_{0},|z|<1$.

En lo que sigue, construimos una solución general en forma cerrada de la ecuación diferencial matricial hipergeométrica (3.39) bajo las hipótesis: $C+n I$ invertible para todo entero $n,(3.40)$ y (3.47). Notar que

$$
\left.\begin{array}{l}
W_{1}(z)=U_{1}(z), \quad W_{2}(z)=U_{2} z^{I-C}, \quad 0<|z|<1, \quad z \in D_{0} \\
(z)=F(A, B ; C ; z), \quad U_{2}(z)=F(A+I-C, B+I-C ; 2 I-C ; z)
\end{array}\right]
$$

son soluciones de (3.39). Por el teorema 3.2.2 anterior, la pareja $\left\{W_{1}, W_{2}\right\}$ es un conjunto fundamental de soluciones de (3.39) en el dominio

$$
\Omega(\delta)=\left\{z \in D_{0}, \quad 0<|z|<\delta\right\}, \quad \delta<1
$$

FUNC. ESPECIALES Y EC. DIF. MATRICIALES 
si la matriz bloque

$$
S(z)=\left[\begin{array}{ll}
W_{1}(z) & W_{2}(z) \\
W_{1}^{\prime}(z) & W_{2}^{\prime}(z)
\end{array}\right],
$$

es invertible en $\Omega(\delta)$. Por las propiedades del complemento de Schur de una matriz, ver [6], la matriz $S(z)$ dada por (3.56) es invertible si y sólo si

$$
M(z)=W_{2}^{\prime}(z)-W_{1}^{\prime}(z)\left[W_{1}(z)\right]^{-1} W_{2}(z) \quad \text { es invertible. }
$$

Notar que

$$
\begin{aligned}
& M(z)=U_{2}^{\prime}(z) z^{I-C}+U_{2}(z) z^{-C}(I-C)-U_{1}^{\prime}(z)\left[U_{1}(z)\right]^{-1} U_{2}(z) z^{I-C}, \\
& M(z)=\left\{U_{2}^{\prime}(z) z+U_{2}(z)(I-C)-U_{1}^{\prime}(z)\left[U_{1}(z)\right]^{-1} U_{2}(z) z\right\} z^{-C} .
\end{aligned}
$$

Por (3.57), notemos que $M(z)$ es invertible si y sólo si

$$
N(z)=z\left[U_{2}^{\prime}(z)-U_{1}^{\prime}(z)\left[U_{1}(z)\right]^{-1} U_{2}(z)\right]+U_{2}(z)(I-C) \text { es invertible. }
$$

Por las demostraciones del teorema 3.3.1 y del corolario 3.3.1 de este capítulo, sabemos que si $0<\gamma<1$, y $n_{2}$ es un entero positivo tal que

$$
\frac{(\|A+I-C\|+n)(\|B+I-C\|+n)}{(n+1)(n-\|2 I-C\|)}<1+\gamma, \quad n \geq n_{2} \geq \operatorname{máx}(1,\|2 I-C\|),
$$

denotando

$$
\begin{gathered}
L_{2}=\frac{(\|A+I-C\|)_{n_{2}}(\|B+I-C\|)_{n_{2}} \Phi\left(n_{2}\right)}{n_{2} !} \\
\Lambda_{2}=\sum_{n=1}^{n_{2}-1} \frac{(\|A+I-C\|)_{n}(\|B+I-C\|)_{n} \Phi(n)}{n !} .
\end{gathered}
$$

donde

$$
\Phi(n)=\left\|(2 I-C)^{-1}\right\|\left\|(3 I-C)^{-1}\right\| \ldots\left\|(-C+(n+1) I)^{-1}\right\|, \quad n \geq 0,
$$

y $\rho_{3}$ es la solución de

$$
h_{1}\left(\rho_{3}\right)=1-\gamma,
$$

con

$$
h_{1}(x)=\Lambda_{2} x+L_{2} \frac{x}{1-x}, \quad 0 \leq x<1
$$

FUNC. ESPECIALES Y EC. DIF. MATRICIALES 
Si $(1+\gamma) \rho_{4}<\rho_{3}$ entonces $U_{2}(z)=F(A+I-C, B+I-C ; 2 I-C ; z)$ satisface

$$
\left\|U_{2}(z)-I\right\|<1-\gamma \quad y \quad\left\|\left(U_{2}(z)\right)^{-1}\right\| \leq \gamma^{-1}, \quad|z| \leq \rho_{4} .
$$

Además, si $n_{3} \geq n_{2}$ se elige de modo que

$$
\begin{gathered}
\frac{(\|A+I-C\|+n)(\|B+I-C\|+n)}{n(n-\|2 I-C\|)}<1+\gamma, \quad n \geq n_{3}>\operatorname{máx}(1,\|2 I-C\|), \\
\left.\Lambda_{3}=\sum_{n=1}^{n_{3}-1} \frac{(\|A+I-C\|)_{n}(\|B+I-C\|)_{n} \Phi(n)}{(n-1) !},\right] \\
\left.L_{3}=\frac{(\|A+I+C\|)_{n_{3}}(\|B+I-C\|)_{n_{3}} \Phi\left(n_{3}\right)}{\left(n_{3}-1\right) !},\right]
\end{gathered}
$$

por los comentarios previos y la prueba del corolario 3.3.1 anterior se tiene

$$
\left.\begin{array}{c}
\left\|U_{2}(z)-I\right\|<1-\gamma, \quad\left\|\left(U_{2}(z)\right)^{-1}\right\| \leq \gamma^{-1} \quad y \\
\left\|U_{2}^{\prime}(z)\right\| \leq \Lambda_{3}+\frac{L_{3}}{1-\rho_{3}}, \quad|z| \leq \rho_{4} .
\end{array}\right]
$$

Luego, acabamos de establecer el siguiente resultado:

Corolario 3.4.1 . Sean A, B, $C$ matrices en $C^{r \times r}$ satisfaciendo: $C+n I$ es invertible para todo entero $n$, excepto posiblemente el 1 , (3.40) y (3.47), y sea $U_{2}(z)=F(A+I-C, B+I-C ; 2 I-C ; z)$. Sea $0<\gamma<1$ y sean $n_{2}$ y $n_{3}$ enteros positivos verificando (3.59) y (3.64) respectivamente. Si $L_{2}, L_{3}$, $\Lambda_{2}$ y $\Lambda_{3}$ están definidas por (3.60), (3.65), $\rho_{3}$ es la solución de la ecuación (3.62), y $\rho_{4}$ elegido de modo que $(1+\gamma) \rho_{4}<\rho_{3}$, entonces (3.66) se cumple.

Supongamos ahora que $C+n I$ es invertible para todo entero $n$. Notar que $N(z)$ dada por (3.58) está bien definida en el disco $|z| \leq \rho_{1}$ donde $U_{1}(z)=$ $F(A, B ; C ; z)$ es invertible. Además, observar que $N(0)=I-C$ es invertible. Por el lema de perturbación, $N(z)$ es invertible en el dominio $|z|<r \leq \rho_{1}$ donde se verifique

$$
\|N(z)-(I-C)\|<\left\|(I-C)^{-1}\right\|^{-1} .
$$


Escribamos para $|z| \leq \rho_{1}$,

$$
\begin{gathered}
N(z)-N(0)=N(z)-(I-C) \\
=z\left[U_{2}^{\prime}(z)-U_{1}^{\prime}(z)\left[U_{1}(z)\right]^{-1} U_{2}(z)\right]+\left(U_{2}(z)-I\right)(I-C),
\end{gathered}
$$

Notar que dado $0<\varepsilon<1$, tomando $\gamma=1-\varepsilon$ en la prueba del corolario 3.4.1 de este capítulo, considerando $n_{4} \geq$ máx $(1,\|2 I-C\|)$ tal que

$$
\frac{(\|A+I-C\|+n)(\|B+I-C\|+n)}{n(n-\|2 I-C\|)}<2-\varepsilon, \quad n \geq n_{4},
$$

y tomando

$$
\begin{gathered}
\Lambda_{4}=\sum_{n=1}^{n_{4}-1} \frac{(\|A+I-C\|)_{n}(\|B+I-C\|)_{n} \Phi(n)}{(n-1) !}, \\
L_{4}=\frac{(\|A+I-C\|)_{n_{4}}(\|B+I-C\|)_{n_{4}} \Phi\left(n_{4}\right)}{\left(n_{4}-1\right) !} . \\
h_{2}(x)=\Lambda_{4} x+L_{4} \frac{x}{1-x}, \quad 0 \leq x<1,
\end{gathered}
$$

si $\rho_{5}$ es la única solución de la ecuación

$$
h_{2}\left(\rho_{5}\right)=\varepsilon,
$$

tomando $\rho_{6}$ de modo que

$$
(1+\gamma) \rho_{6}=\rho_{6}(2-\varepsilon)<\rho_{5},
$$

entonces

$$
\left\|U_{2}(z)-I\right\|<\varepsilon, \quad|z| \leq \rho_{6} .
$$

Usando el teorema 3.3.1 de este capítulo, los corolarios 3.3.1, 3.4.1 anteriores y por (3.62)-(3.74), tomando $|z|<\operatorname{mín}\left(\rho_{1}, \rho_{4}, \rho_{6}\right)$ se deduce que

$$
\begin{gathered}
\|N(z)-N(0)\| \leq|z|\left[\left\|U_{2}^{\prime}(z)\right\|+\left\|\left(U_{1}(z)\right)^{-1}\right\|\left\|U_{1}^{\prime}(z)\right\|\left\|U_{2}(z)\right\|\right] \\
+\left\|U_{2}(z)-I\right\|\|I-C\| \\
\leq|z|\left[\left(\Lambda_{3}+\frac{L_{3}}{1-\rho_{3}}\right)+\left(\frac{1+\varepsilon}{1-\varepsilon}\right)\left(\Lambda_{1}+\frac{L_{1}}{1-\rho_{1}}\right)\right]+\varepsilon\|I-C\|,
\end{gathered}
$$


Luego, tomando

$$
\varepsilon=\frac{1}{2} \min \left\{\left\|(I-C)^{-1}\right\|^{-1}(\|I-C\|)^{-1}, \quad 1\right\},
$$

y $|z|<\operatorname{mín}\left(\rho_{1}, \rho_{4}, \rho_{6}, \rho_{7}\right)=\rho^{*}$, donde

$$
\rho_{7}=\frac{1}{2}\left\{\left[\left(\Lambda_{3}+\frac{L_{3}}{1-\rho_{3}}\right)+\left(\frac{1+\varepsilon}{1-\varepsilon}\right)\left(\Lambda_{1}+\frac{L_{1}}{1-\rho_{1}}\right)\right]\left(\left\|(I-C)^{-1}\right\|\right)\right\}^{-1}
$$

por (3.75) se tiene

$$
\|N(z)-N(0)\|<\left\|(I-C)^{-1}\right\|^{-1}, \quad|z|<\rho^{*},
$$

y por tanto

$$
N(z) \text { es invertible para }|z|<\operatorname{mín}\left(\rho_{1}, \rho_{4}, \rho_{6}, \rho_{7}\right)=\rho^{*} .
$$

Resumiendo, por los teoremas 3.2.1 y 3.3.1, los corolarios 3.3.1 y 3.4.1 anteriores, y por los comentarios previos, acabamos de demostrar el siguiente resultado:

Teorema 3.4.1 . Sean $A, B, C$ matrices en $C^{r \times r}$ que verifican las propiedades: $C+n I$ invertible para todo entero $n,(3.40)$ y (3.47), y sean $W_{1}(z)$ y $W_{2}(z)$ las definidas por (3.55) para $z$ en $D_{0}$ con $|z|<1$. Entonces existe un número positivo $\rho^{*}<1$ tal que la solución general de la ecuación (3.39) en $\Omega\left(\rho^{*}\right)=\left\{z \in D_{0} ; \quad 0<|z|<\rho^{*}\right\}$ está dada por

$$
W(z)=W_{1}(z) P+W_{2}(z) Q, \quad P, Q \in C^{r \times r} .
$$

Además, $\rho^{*}$ puede ser determinado de acuerdo con el siguiente procedimiento:

- Sea el número positivo $\varepsilon=\frac{1}{2} \operatorname{mín}\left\{1,\left\|(I-C)^{-1}\right\|^{-1}\|I-C\|^{-1}\right\}$, toma$\operatorname{mos} \gamma=1-\varepsilon$.

- Sea $n_{0}$ un entero positivo cumpliendo la desigualdad dada en (3.22).

- Sea $n_{1}$ definido por la inecuación (3.33) y $\Lambda_{1}, L_{1}$ los números reales positivos definidos por (3.34).

FUNC. ESPECIALES Y EC. DIF. MATRICIALES 
- Sea $\rho_{0}$ la solución de la ecuación (3.25) donde f está definida por (3.24) con $\Lambda$ y L los escalares dados por (3.23). Tomamos $\rho_{1}$ de manera que $\rho_{1}<$ $(1+\gamma)^{-1} \rho_{0}$.

- Sea $n_{2}$ elegido de modo que (3.59) se cumpla y sean $\Lambda_{2}, L_{2}$ los definidos por (3.60). Sea $\rho_{3}$ la solución de (3.62) con $h_{1}$ dada por (3.63). Tomamos $\rho_{4}$ con $\rho_{4}<(1+\gamma)^{-1} \rho_{3}$.

- Sea $n_{3}$ definido de forma que (3.64) se verifique, y sean $\Lambda_{3}, L_{3}$ los números reales positivos definidos por (3.65).

- Tomemos $n_{4}$ de manera que (3.69) se cumpla y sean $\Lambda_{4}, L_{4}$ los definidos por las igualdades (3.70). Si $\rho_{5}$ es la solución de la ecuación (3.72) donde la función $h_{2}$ está definida por (3.71), tomemos $\rho_{6}$ de forma que verifique la desigualdad $\rho_{6}<(1+\gamma)^{-1} \rho_{5}$.

- Sea $\rho_{7}$ definido por la expresión (3.77), entonces basta tomar $\rho^{*}$ del siguiente modo, $\rho^{*}=\operatorname{mín}\left\{\rho_{1}, \rho_{4}, \rho_{6}, \rho_{7}\right\}$.

De esta forma queda determinada la solución general de la ecuación diferencial hipergeométrica matricial en un dominio que además es posible calcular en la práctica, siguiendo el algoritmo que acabamos de describir.

\subsection{Estudio de la función hipergeométrica ma- tricial en la frontera de su recinto de con- vergencia}

Analizamos en este quinto apartado qué sucede en la frontera del dominio de convergencia de la función hipergeométrica matricial $F(A, B ; C ; z)$, es decir, en la circunferencia de centro 0 y radio $1,|z|=1$. Concretamente, estableceremos una condición suficiente para garantizar que la serie matricial que define a $F(A, B ; C ; z)$ converja en $|z|=1$. Esta condición se impondrá sobre las matrices $A, B$ y $C$.

Con esta finalidad, empezamos dando la siguiente:

FUNC. ESPECIALES Y EC. DIF. MATRICIALES 
Definición 3.5.1 . Dada $M \in C^{r \times r}$, y siendo $\sigma(M)$ el espectro de $M$, denotaremos por $\alpha(M)$ y $\beta(M)$ a los números

$$
\left.\begin{array}{l}
\alpha(M)=\operatorname{máx}\{\operatorname{Re}(\lambda): \lambda \in \sigma(M)\} \\
\beta(M)=\operatorname{mín}\{\operatorname{Re}(\lambda): \lambda \in \sigma(M)\}
\end{array}\right]
$$

Supongamos que $A, B$ y $C$ son como en (2.8) del capítulo 2, es decir, cumpliendo

$$
\operatorname{Re}(\lambda)>0 \quad \forall \lambda \in \sigma(A), \sigma(B), \sigma(C),
$$

y tales que verifican la siguiente desigualdad

$$
\beta(C)-\alpha(A)-\alpha(B)>0
$$

y llamemos

$$
\delta=\frac{1}{2}[\beta(C)-\alpha(A)-\alpha(B)]>0
$$

Para probar que la serie matricial que define la función hipergeométrica matricial

$$
F(A, B ; C ; z)=\sum_{n \geq 0} \frac{1}{n !}(A)_{n}(B)_{n}(C)_{n}^{-1} z^{n}
$$

converge absolutamente en la circunferencia unidad, i.e. $|z|=1$, la compararemos con la serie numérica de términos positivos

$$
\sum_{n \geq 1} \frac{1}{n^{1+\delta}}
$$

(la cual sabemos que es convergente, pues $\delta>0$ ), usando el siguiente resultado sobre series numéricas:

Lema 3.5.1. Sean $\sum_{n \geq 0} a_{n} y \sum_{n \geq 0} b_{n}$ series de términos positivos, siendo $\sum_{n \geq 0} b_{n}$ convergente, $y$ de modo que $\lim _{n \rightarrow \infty} \frac{a_{n}}{b_{n}}=0$, entonces la serie $\sum_{n \geq 0} a_{n}$ converge. 
Tomando

$$
a_{n}=\left\|\frac{1}{n !}(A)_{n}(B)_{n}(C)_{n}^{-1} z^{n}\right\|,
$$

y

$$
b_{n}=\frac{1}{n^{1+\delta}}
$$

si demostramos que

$$
\lim _{n \rightarrow \infty} n^{1+\delta}\left\|\frac{1}{n !}(A)_{n}(B)_{n}(C)_{n}^{-1} z^{n}\right\|=0,
$$

aplicando el criterio de comparación de series anterior, habremos probado lo que queremos.

Veamos (3.82), demostrando las dos desigualdades que determinan la igualdad deseada.

Por una parte, es claro que

$$
\lim _{n \rightarrow \infty} n^{1+\delta}\left\|\frac{1}{n !}(A)_{n}(B)_{n}(C)_{n}^{-1} z^{n}\right\| \geq 0
$$

Veamos ahora la desigualdad contraria, es decir, veamos que también se cumple que

$$
\lim _{n \rightarrow \infty} n^{1+\delta}\left\|\frac{1}{n !}(A)_{n}(B)_{n}(C)_{n}^{-1} z^{n}\right\| \leq 0
$$

Para ello, y como veremos a continuación, realizaremos distintas manipulaciones con el fin de aplicar la representación a través de un límite de la función Gamma de una matriz cuadrada, demostrada en el capítulo segundo, y que recordamos a continuación

$$
\Gamma(M)=\lim _{n \rightarrow \infty}(n-1) !(M)_{n} n^{M}
$$

siendo $M$ una matriz de $C^{r \times r}$ que satisface

$$
\operatorname{Re}(z)>0 \quad \forall z \in \sigma(M)
$$

y $n \geq 1$ un entero, y que aplicaremos a los coeficientes matriciales $A, B$ y $C$.

FUNC. ESPECIALES Y EC. DIF. MATRICIALES 
En efecto

$$
\begin{gathered}
\lim _{n \rightarrow \infty} n^{1+\delta}\left\|\frac{1}{n !}(A)_{n}(B)_{n}(C)_{n}^{-1} z^{n}\right\| \\
=\lim _{n \rightarrow \infty}\left\|\frac{n^{1+\delta}(A)_{n}(B)_{n}(C)_{n}^{-1} z^{n}}{n !}\right\| \\
=\lim _{n \rightarrow \infty} \| \frac{n^{1+\delta}}{n !} \cdot \frac{(n-1) ! n^{A} n^{-A}(A)_{n}}{(n-1) !} \cdot \\
\cdot \frac{(n-1) ! n^{B} n^{-B}(B)_{n}}{(n-1) !} \cdot \frac{(n-1) !(C)_{n}^{-1} n^{C} n^{-C}}{(n-1) !} z^{n} \| \\
=\| \lim _{n \rightarrow \infty}\left[\frac{n^{1+\delta}}{n} \cdot n^{A} \cdot \frac{n^{-A}(A)_{n}}{(n-1) !}\right. \\
\left.\cdot n^{B} \frac{n^{-B}(B)_{n}}{(n-1) !}(n-1) !(C)_{n}^{-1} n^{C} n^{-C} z^{n}\right] \| \\
=\lim _{n \rightarrow \infty}\left(\frac{n^{1+\delta} n^{A}}{n}\right) \lim _{n \rightarrow \infty}\left(\frac{n^{-A}(A)_{n}}{(n-1) !}\right) \lim _{n \rightarrow \infty}\left(n^{B}\right) \lim _{n \rightarrow \infty}\left(\frac{n^{-B}(B)_{n}}{(n-1) !}\right) \\
\lim _{n \rightarrow \infty}\left((n-1) !(C)_{n}^{-1} n^{C}\right) \lim _{n \rightarrow \infty}\left(n^{-C} z^{n}\right) \|=
\end{gathered}
$$

usando el teorema 2.2.1 sobre las matrices $A, B$ y $C$ continuamos con la última igualdad

$$
=\lim _{n \rightarrow \infty}\left\|n^{\delta} n^{A} \Gamma^{-1}(A) n^{B} \Gamma^{-1}(B) \Gamma(C) n^{-C} z^{n}\right\| \leq
$$

aplicando la propiedad submultiplicativa de la norma matricial y que $|z|=1$, proseguimos con la última desigualdad

$$
\leq \lim _{n \rightarrow \infty}\left\|\Gamma^{-1}(A)\right\|\left\|\Gamma^{-1}(B)\right\|\|\Gamma(C)\| \lim _{n \rightarrow \infty}\left[n^{\delta}\left\|n^{A}\right\|\left\|n^{B}\right\|\left\|n^{-C}\right\|\right] \leq
$$


usando la expresión (2.7) del capítulo 2, deducimos que

$$
\begin{gathered}
\left\|n^{A}\right\| \leq n^{\alpha(A)}\left(\sum_{k=0}^{r-1} \frac{(\|A\| \sqrt{r} \ln n)^{k}}{k !}\right) \\
\left\|n^{B}\right\| \leq n^{\alpha(B)}\left(\sum_{k=0}^{r-1} \frac{(\|B\| \sqrt{r} \ln n)^{k}}{k !}\right) \\
\left\|n^{-C}\right\| \leq n^{-\beta(C)}\left(\sum_{k=0}^{r-1} \frac{(\|C\| \sqrt{r} \ln n)^{k}}{k !}\right)
\end{gathered}
$$

notar que en esta cota de $\left\|n^{-C}\right\|$ hemos usado que $\alpha(-C)=-\beta(C)$. Así, haciendo uso de (3.86) en (3.85) proseguimos con la acotación

$$
\begin{gathered}
\leq\left\|\Gamma^{-1}(A)\right\|\left\|\Gamma^{-1}(B)\right\|\|\Gamma(C)\| \cdot \\
\cdot \lim _{n \rightarrow \infty}\left[n^{\delta+\alpha(A)+\alpha(B)-\beta(C)}\left(\sum_{k=0}^{r-1} \frac{(\|A\| \sqrt{r} \ln n)^{k}}{k !}\right) .\right. \\
\left.\cdot\left(\sum_{k=0}^{r-1} \frac{(\|B\| \sqrt{r} \ln n)^{k}}{k !}\right)\left(\sum_{k=0}^{r-1} \frac{(\|C\| \sqrt{r} \ln n)^{k}}{k !}\right)\right]=
\end{gathered}
$$

de (3.81) se deduce

$$
\delta+\alpha(A)+\alpha(B)-\beta(C)=-\delta
$$

que sustituido en la última acotación nos conduce a

$$
\begin{gathered}
=\left\|\Gamma^{-1}(A)\right\|\left\|\Gamma^{-1}(B)\right\|\|\Gamma(C)\| \cdot \\
\cdot \lim _{n \rightarrow \infty}\left[n^{-\delta}\left(\sum_{k=0}^{r-1} \frac{(\|A\| \sqrt{r} \ln n)^{k}}{k !}\right) \cdot\right. \\
\left.\cdot\left(\sum_{k=0}^{r-1} \frac{(\|B\| \sqrt{r} \ln n)^{k}}{k !}\right)\left(\sum_{k=0}^{r-1} \frac{(\|C\| \sqrt{r} \ln n)^{k}}{k !}\right)\right]=0
\end{gathered}
$$

FUNC. ESPECIALES Y EC. DIF. MATRICIALES 
y como por L'Hôpital

$$
\lim _{n \rightarrow \infty} n^{-\delta}(\ln n)^{r}=0
$$

se deduce que el último límite es efectivamente cero como hemos escrito en la última igualdad.

Así pues, hemos probado que

$$
\lim _{n \rightarrow \infty} n^{1+\delta}\left\|\frac{1}{n !}(A)_{n}(B)_{n}(C)_{n}^{-1} z^{n}\right\| \leq 0
$$

De (3.83) y de (3.87), se deduce

$$
\lim _{n \rightarrow \infty} n^{1+\delta}\left\|\frac{1}{n !}(A)_{n}(B)_{n}(C)_{n}^{-1} z^{n}\right\|=0
$$

Resumiendo, acabamos de establecer el siguiente resultado:

Teorema 3.5.1 . Sean $A, B$ y $C$ matrices en $C^{r \times r}$ de modo que se cumple $\operatorname{Re}(\lambda)>0, \quad \forall \lambda \in \sigma(A), \quad \sigma(B), \quad \sigma(C)$ y usando la notación de (3.80) supongamos que $\beta(C)-\alpha(A)-\alpha(B)>0$, entonces

$$
F(A, B ; C ; z)=\sum_{n \geq 0} \frac{1}{n !}(A)_{n}(B)_{n}(C)_{n}^{-1}
$$

converge en la frontera $|z|=1$

\subsection{Una representación integral para la fun- ción hipergeométrica matricial}

El propósito de este último apartado es, utilizando la relación que se dedució en el segundo capítulo entre las funciones Beta y Gamma matriciales, dar una representación integral sencilla de la función hipergeométrica matricial $F(A, B ; C ; z)$, en la cual veremos que interviene la función Gamma matricial.

Comenzamos desarrollando una serie de resultados previos que luego aplicaremos.

FUNC. ESPECIALES Y EC. DIF. MATRICIALES 
Por el teorema binomial escalar, sabemos

$$
(1+x)^{t}=\sum_{n=0}^{\infty} \frac{t(t-1) \ldots(t-n+1)}{n !} x^{n}, \quad|x|<1,
$$

si lo aplicamos para $x=-y$ y $t=-a$, obtenemos

$$
\begin{aligned}
& (1-y)^{-a}=\sum_{n=0}^{\infty} \frac{(-a)(-a-1) \ldots(-a-n+1)}{n !}(-y)^{n} \\
& =\sum_{n=0}^{\infty} \frac{(a)(a+1) \ldots(a+n-1)}{n !} y^{n}=\sum_{n=0}^{\infty} \frac{(a)_{n}}{n !} y^{n} .
\end{aligned}
$$

notar que en el último paso hemos introducido la función factorial escalar $(z)_{n}=z(z+1) \ldots(z+n-1), \quad n>0, \mathrm{y}(z)_{0}=1$, con $z \in C$. Así pues, tenemos que

$$
(1-y)^{-a}=\sum_{n=0}^{\infty} \frac{(a)_{n}}{n !} y^{n}, \quad|y|<1,
$$

con lo que aplicando el cálculo funcional holomorfo se tiene que

$$
(1-y)^{-A}=\sum_{n=0}^{\infty} \frac{(A)_{n}}{n !} y^{n}, \quad|y|<1, \quad A \in C^{r \times r} .
$$

Justifiquemos formalmente esta extensión de la fórmula escalar a la fórmula matricial por el cálculo funcional holomorfo, y para ello recordemos que por el teorema de Weierstrass, dada $\left\{f_{n}\right\}$ una sucesión de funciones holomorfas en $\Omega$, siendo $\Omega \subseteq C$ abierto, de modo que en cualquier disco $D$ cerrado y acotado, y contenido en $\Omega$, si la serie de funciones $\sum_{n \geq 0} f_{n}(z)$ converge uniformemente en $D$, entonces la función $f(z)=\sum_{n \geq 0} f_{n}(z)$ es holomorfa en $\Omega$.

Sea $y \in C$ fijo, con $|y|<1$, y sea $a \in C$. Definamos a continuación la función compleja de variable compleja

$$
f(a)=(1-y)^{-a}=\sum_{n \geq 0} f_{n}(a), \quad f_{n}(a)=\frac{(a)_{n}}{n !} y^{n}
$$

entonces, $\left\{f_{n}(a)\right\}$ es una sucesión de funciones holomorfas en el conjunto abierto $\Omega=C$. Además, dado $R>0$, arbitrario pero fijo, consideremos el

FUNC. ESPECIALES Y EC. DIF. MATRICIALES 
disco cerrado y acotado $D=\{a \in C /|a| \leq R\}$, contenido en $\Omega=C$, entonces como

$$
\left|f_{n}(a)\right| \leq \frac{1}{n !}(|a|)_{n}|y|^{n} \leq \frac{1}{n !}(R)_{n}|y|^{n} \quad \forall a \in D
$$

$\mathrm{y}$

$$
\sum_{n \geq 0} \frac{1}{n !}(R)_{n}|y|^{n}<\infty
$$

pues por el criterio del D'Alembert

$$
\lim _{n \rightarrow \infty} \frac{n !(R)_{n+1}|y|^{n+1}}{(n+1) !(R)_{n}|y|^{n}}=\lim _{n \rightarrow \infty} \frac{(R+n)|y|}{n+1}=|y|<1
$$

aplicando el criterio de mayoración de Weierstrass, se deduce que $\sum_{n \geq 0} f_{n}(a)$ converge uniformemente en $D$, y por tanto por el teorema de Weierstrass obtenemos que

$$
f(a)=(1-y)^{-a}=\sum_{n \geq 0} \frac{(a)_{n}}{n !} y^{n}
$$

es holomorfa en $\Omega=C$, así aplicando el cálculo funcional holomorfo, concluimos que

$$
f(A)=(1-y)^{-A}=\sum_{n \geq 0} \frac{(A)_{n}}{n !} y^{n}, \quad|y|<1, \quad A \in C^{r \times r} .
$$

Más adelante, necesitaremos conmutar una integral con una serie de funciones, para ello, recordamos ahora las condiciones que nos permiten realizar este paso, dando la siguiente

Definición 3.6.1 . Una serie $\sum_{n \geq 0} u_{n}(t)$ se dice acotadamente convergente en el intervalo $[a, b]$ si y sólo si dicha serie converge para cualquier valor $t \in[a, b]$, y existe una constante positiva $M$, tal que $\left|S_{n}(t)\right| \leq M \quad \forall n \geq 0$ y $\forall t \in[a, b]$, siendo $S_{n}(t)=\sum_{k=0}^{n} u_{k}(t)$.

Sabemos por [63] que una condición suficiente para integrar la serie $\sum_{n \geq 0} u_{n}(t)$ término a término en el intervalo $[a, b]$ es que $\sum_{n \geq 0} u_{n}(t)$ sea uniformemente 
convergente y acotadamente convergente en $[a, b]$.

Como se ha señalado anteriormente, próximamente necesitaremos hacer la siguiente integración término a término:

$$
\begin{aligned}
& \int_{0}^{1}\left\{\sum_{n \geq 0}\left(\frac{1}{n !}(A)_{n}(t z)^{n}\right) t^{B-I}(1-t)^{C-B-I} d t\right\} \\
= & \sum_{n \geq 0}\left\{\int_{0}^{1}\left(\frac{1}{n !}(A)_{n}(t z)^{n}\right) t^{B-I}(1-t)^{C-B-I} d t\right\}
\end{aligned}
$$

donde $t \in[0,1], z \in C$, tal que $|z|<1$, y $A, B, C \in C^{r \times r}$, de modo que $B$ y $C$ son matrices diagonalizables verificando (3.40) y

$$
\begin{gathered}
\min \{\operatorname{Re}(c-b) / b \in \sigma(B), c \in \sigma(C)\}>0 \quad \forall b \in \sigma(B), \forall c \in \sigma(C), \\
\operatorname{Re}(b)>0, \operatorname{Re}(c)>0 .
\end{gathered}
$$

Justifiquemos ahora el paso dado en (3.89) sobre la conmutación de la integral con la suma infinita. Para ello según hemos visto antes es suficiente probar que la serie

$$
\sum_{n \geq 0} u_{n}(t) \quad \text { con } \quad u_{n}(t)=\frac{1}{n !}(A)_{n}(t z)^{n} t^{B-I}(1-t)^{C-B-I}
$$

es acotadamente convergente en $[0,1]$ y uniformemente convergente en $[0,1]$. Sea $z \in C$ con $|z|<1$ fijo, y sea $t \in[0,1]$ arbitrario, entonces

$$
\left.\begin{array}{c}
\left\|S_{n}(t)\right\|=\left\|\sum_{i=0}^{n} \frac{1}{i !}(A)_{i}(t z)^{i} t^{B-I}(1-t)^{C-B-I}\right\| \\
\leq \sum_{i=0}^{n} \frac{1}{i !}(\|A\|)_{i}(|t z|)^{i}\left\|t^{B-I}\right\|\left\|(1-t)^{C-B-I}\right\| \leq
\end{array}\right]
$$


notar que por (2.7) sabemos

$$
\left.\begin{array}{c}
\left\|t^{B-I}\right\| \leq t^{\alpha(B)-1}\left(\sum_{j=0}^{r-1} \frac{(\|B-I\| \sqrt{r} \ln t)^{j}}{j !}\right) \\
\left\|t^{C-B-I}\right\| \leq t^{\alpha(C-B)-1}\left(\sum_{j=0}^{r-1} \frac{(\|C-B-I\| \sqrt{r} \ln t)^{j}}{j !}\right)
\end{array}\right]
$$

donde hemos usado que

$$
\begin{gathered}
\alpha(B-I)=\alpha(B)-1 \\
\alpha(B-C-I)=\alpha(B-C)-1
\end{gathered}
$$

Aplicando (3.92) en (3.91), y que

$$
\ln t \leq t, \quad \ln (1-t) \leq 1-t, \quad t \in(0,1)
$$


continuamos con la última desigualdad

$$
\begin{aligned}
& \leq \sum_{i=0}^{n} \frac{1}{i !}(\|A\|)_{i}|z|^{i} t^{\alpha(B)-1}(1-t)^{\alpha(C-B)-1} . \\
& \cdot\left(\sum_{m=0}^{r-1} \frac{(\|B-I\| \sqrt{r} t)^{m}}{m !}\right)\left(\sum_{l=0}^{r-1} \frac{(\|C-B-I\| \sqrt{r}(1-t))^{l}}{l !}\right) \\
& =\sum_{i=0}^{n} \frac{1}{i !}(\|A\|)_{i}|z|^{i} t^{\alpha(B)-1}(1-t)^{\alpha(C-B)-1} . \\
& \cdot\left(\sum_{h=0}^{2(r-1)} \sum_{m+l=h} \frac{(\|B-I\| \sqrt{r} t)^{m}}{m !} \cdot \frac{(\|C-B-I\| \sqrt{r}(1-t))^{l}}{l !}\right) \\
& =\sum_{i=0}^{n} \frac{1}{i !}(\|A\|)_{i}|z|^{i} t^{\alpha(B)-1}(1-t)^{\alpha(C-B)-1} . \\
& \cdot\left(\sum_{h=0}^{2(r-1)} \sum_{m+l=h} \frac{\|B-I\|^{m}(\sqrt{r})^{m} t^{m}\|C-B-I\|^{l}(\sqrt{r})^{l}(1-t)^{l}}{m ! l !}\right) \\
& =\sum_{i=0}^{n} \frac{1}{i !}(\|A\|)_{i}|z|^{i} . \\
& \cdot\left\{\sum_{h=0}^{2(r-1)} \sum_{m+l=h} \frac{\|B-I\|^{m}\|C-B-I\|^{l}(\sqrt{r})^{h}}{m ! l !} t^{\alpha(B)+m-1}(1-t)^{\alpha(C-B)+l-1}\right\}=\varphi(t)
\end{aligned}
$$


Observemos ahora que

$$
\begin{gathered}
\int_{0}^{1} \varphi(t) d t \\
=\int_{0}^{1}\left(\sum _ { i = 0 } ^ { n } \frac { 1 } { i ! } ( \| A \| ) _ { i } | z | ^ { i } \left\{\sum_{h=0}^{2(r-1)} \sum_{m+l=h} \frac{\|B-I\|^{m}\|C-B-I\|^{l}(\sqrt{r})^{h}}{m ! l !}\right.\right. \\
\left.\left.\cdot t^{\alpha(B)+m-1} \cdot(1-t)^{\alpha(C-B)+l-1}\right\}\right) d t \\
=\left\{\sum_{i=0}^{n} \frac{1}{i !}(\|A\|)_{i}|z|^{i} \cdot\right. \\
\sum_{h=0}^{2(r-1)} \sum_{m+l=h} \frac{\|B-I\|^{m}\|C-B-I\|^{l}(\sqrt{r})^{h}}{m ! l !} \\
\left.\cdot \int_{0}^{1} t^{\alpha(B)+m-1}(1-t)^{\alpha(C-B)+l-1} d t\right\}=
\end{gathered}
$$

notar que por (3.90)

$$
\begin{gathered}
\operatorname{Re}(\alpha(B)+m)=\alpha(B)+m>0 \\
\operatorname{Re}(\alpha(C-B)+l)=\alpha(C-B)+l>0
\end{gathered}
$$

así

$$
\int_{0}^{1} t^{\alpha(B)+m-1}(1-t)^{\alpha(C-B)+l-1} d t=B(\alpha(B)+m, \alpha(C-B)+l)
$$

luego continuando con la última igualdad de (3.93)

$$
\begin{gathered}
=\sum_{i=0}^{n} \frac{1}{i !}(\| A \mid)_{i}|z|^{i} . \\
\cdot\left\{\sum_{h=0}^{2(r-1)} \sum_{m+l=h} \frac{\|B-I\|^{m}\|C-B-I\|^{l}(\sqrt{r})^{h}}{m ! l !} \cdot B(\alpha(B)+m, \alpha(C-B)+l)\right\}=
\end{gathered}
$$


denotando por

$$
H=\left\{\sum_{h=0}^{2(r-1)} \sum_{m+l=h} \frac{\|B-I\|^{m}\|C-B-I\|^{l}(\sqrt{r})^{h}}{m ! l !} \cdot B(\alpha(B)+m, \alpha(C-B)+l)\right\}
$$

podemos continuar la última igualdad

$$
=H \sum_{i=0}^{n} \frac{1}{i !}(\|A\|)_{i}|z|^{i}<H \sum_{i=0}^{\infty} \frac{1}{i !}(\|A\|)_{i}|z|^{i}<\infty
$$

Para ver la convergencia de la última serie hemos utilizado el criterio de D'Alembert

$$
\lim _{i \rightarrow \infty} \frac{i !(\|A\|)_{i+1}|z|^{i+1}}{(i+1) !(\|A\|)_{i}|z|^{i}}=\lim _{i \rightarrow \infty} \frac{(\|A\|+i)}{i+1}|z|=|z|<1
$$

Este proceso sirve para probar también que la serie

$$
\sum_{n \geq 0} \frac{1}{n !}(A)_{n}(t z)^{n} t^{B-I}(1-t)^{C-B-I}
$$

converge uniformemente en $[0,1]$, por aplicación del criterio de Weierstrass. Por lo tanto, dicha serie es uniformemente convergente en $[0,1]$, y acotadamente convergente en $[0,1]$, por lo que podemos integrarla término a término en dicho intervalo.

Nos dirigimos ya al propósito de este apartado. Recordemos que estamos trabajando bajo las hipótesis: $B$ y $C$ matrices diagonalizables, que cumplen (3.40) y (3.90).

Dadas $B, C \in C^{r \times r}$ consideremos las matrices $P=B+n I, Q=C-B$, entonces como

- $R e(z)>0, \forall z \in \sigma(B+n I)$, ya que, por [24, p.54] podemos escribir que $\sigma(B+n I)=\{b+n\}$ con $b \in \sigma(B)$, y por $(3.90) \operatorname{Re}(b)>0$.

- $\operatorname{Re}(z)>0, \forall z \in \sigma(C-B)$, ya que, igual que antes por [24, p.54] se deduce que $\sigma(C-B)=\{c-b\}$ con $b \in \sigma(B)$ y $c \in \sigma(C)$ y por (3.90)

$$
\text { mín }\{\operatorname{Re}(c-b) \quad / b \in \sigma(B), c \in \sigma(C)\}>0 .
$$

FUNC. ESPECIALES Y EC. DIF. MATRICIALES 
- $P$ y $Q$ son diagonalizables, por serlo $B$ y $C$ por hipótesis.

- $P$ y $Q$ conmutan, por conmutar por hipótesis $B$ y $C$.

por lo que aplicando el teorema 2.2.2 del capítulo 2 sabemos

$$
\left.\begin{array}{c}
\Gamma(C-B) \Gamma(B+n I) \Gamma^{-1}(C+n I) \\
=B(B+n I, C-B)=\int_{0}^{1} t^{B+(n-1) I}(1-t)^{C-B-I} d t
\end{array}\right]
$$

Aplicando (2.4)

$$
\begin{gathered}
(B)_{n}(C)_{n}^{-1}=\Gamma^{-1}(B) \Gamma(B+n I)\left[\Gamma^{-1}(C) \Gamma(C+n I)\right]^{-1} \\
=\Gamma^{-1}(B) \Gamma(B+n I) \Gamma^{-1}(C+n I) \Gamma(C) \\
=\Gamma^{-1}(B) \Gamma^{-1}(C-B) \Gamma(C-B) \Gamma(B+n I) \Gamma^{-1}(C+n I) \Gamma(C)=
\end{gathered}
$$

usando (3.94) continuamos con la última igualdad

$$
=\Gamma^{-1}(B) \Gamma^{-1}(C-B)\left(\int_{0}^{1} t^{B+(n-1) I}(1-t)^{C-B-I} d t\right) \Gamma(C)
$$

Así pues, hemos deducido que

$$
(B)_{n}(C)_{n}^{-1}=\Gamma^{-1}(B) \Gamma^{-1}(C-B)\left(\int_{0}^{1} t^{B+(n-1) I}(1-t)^{C-B-I} d t\right) \Gamma(C)
$$

Por otra parte para $|z|<1$, sabemos que

$$
F(A, B ; C ; z)=\sum_{n \geq 0} \frac{1}{n !}(A)_{n}(B)_{n}(C)_{n}^{-1} z^{n}=
$$


aplicando (3.95),

$$
\begin{aligned}
& =\sum_{n \geq 0} \frac{1}{n !}(A)_{n} \Gamma^{-1}(B) \Gamma^{-1}(C-B)\left(\int_{0}^{1} t^{B+(n-1) I}(1-t)^{C-B-I} d t\right) \Gamma(C) z^{n} \\
& =\sum_{n \geq 0}\left\{\int_{0}^{1} \frac{1}{n !}(A)_{n} \Gamma^{-1}(B) \Gamma^{-1}(C-B) t^{B+(n-1) I}(1-t)^{C-B-I} \Gamma(C) z^{n} d t\right\} \\
& =\sum_{n \geq 0}\left\{\int_{0}^{1} \frac{1}{n !}(A)_{n} \Gamma^{-1}(B) \Gamma^{-1}(C-B) t^{B-I} t^{n}(1-t)^{C-B-I} \Gamma(C) z^{n} d t\right\} \\
& =\sum_{n \geq 0}\left\{\int_{0}^{1} \frac{1}{n !}(A)_{n} \Gamma^{-1}(B) \Gamma^{-1}(C-B) t^{B-I}(1-t)^{C-B-I}(t z)^{n} \Gamma(C) d t\right\}=
\end{aligned}
$$

continuamos con la última igualdad, en un primer paso, agrupando términos, y después permutando el sumatorio con la integral en virtud de (3.89)

$$
\begin{aligned}
& =\sum_{n \geq 0}\left\{\int_{0}^{1} \frac{1}{n !}(A)_{n}(t z)^{n} t^{B-I}(1-t)^{C-B-I} d t\right\} \Gamma^{-1}(B) \Gamma^{-1}(C-B) \Gamma(C) \\
= & \int_{0}^{1}\left\{\sum_{n \geq 0}\left(\frac{1}{n !}(A)_{n}(t z)^{n}\right) t^{B-I}(1-t)^{C-B-I}\right\} d t \Gamma^{-1}(B) \Gamma^{-1}(C-B) \Gamma(C)=
\end{aligned}
$$

usando (3.88), pues como $t \in[0,1]$ y $|z|<1$, entonces $|t z|<1$ podemos continuar con la última igualdad

$$
=\int_{0}^{1}(1-t z)^{-A} t^{B-I}(1-t)^{C-B-I} d t \cdot \Gamma^{-1}(B) \Gamma^{-1}(C-B) \Gamma(C)
$$

Resumiendo, acabamos de establecer la siguiente representación integral de la función hipergeométrica matricial

Teorema 3.6.1 . Sean B y $C$ matrices diagonalizables satisfaciendo las condiciones (3.40) y (3.90), sea $A \in C^{r \times r}, z \in C$ de modo que $|z|<1$, entonces

$$
F(A, B ; C ; z)=\int_{0}^{1}(1-t z)^{-A} t^{B-I}(1-t)^{C-B-I} d t \cdot \Gamma^{-1}(B) \Gamma^{-1}(C-B) \Gamma(C)
$$




\section{Parte III}

\section{LAS ECUACIONES DIFERENCIALES MATRICIALES DE SYLVESTER Y DE RICCATI}




\section{Capítulo 4}

\section{SOBRE LA ECUACION DIFERENCIAL MATRICIAL DE SYLVESTER CON COEFICIENTES VARIABLES}

\subsection{Introducción}

Las ecuaciones diferenciales matriciales de Sylvester del tipo

$$
X^{\prime}(t)=A(t) X(t)+X(t) B(t)+F(t), \quad X(0)=C,
$$

donde los coeficientes $A(t), B(t)$ y $F(t)$, así como la incógnita $X(t)$ son matrices en $C^{r \times r}$ aparecen con frecuencia en estructuras flexibles de superespacios [4], sistemas lineales con impulsos [48], sistemas lineales de control con cambios modales no markovianos [61], o cuando se usa la técnica de semidiscretización para resolver ecuaciones en derivadas parciales escalares [54]. Para el caso particular en que los coeficientes son matrices reales y $B(t)$ es la matriz transpuesta de $A(t)$, la ecuación (4.1) se transforma en una ecuación diferencial de Lyapunov. En [20], pueden encontrarse una gran variedad de aplicaciones, propiedades y ejemplos de ecuaciones diferenciales de Lyapunov.

En [58], [25], [5], [12], [31], se dan varios métodos de integración numérica para resolver el problema (4.1) para el caso en que $A(t), B(t)$ y $F(t)$ 
son matrices constantes. Una modificación del método de Runge-Kutta para el problema (4.1) está propuesta en [60]. Además, un método para la construcción de soluciones numéricas continuas de (4.1) fue recientemente dado en [34], usando problemas asociados unilaterales lineales y métodos matriciales de un paso. Sin embargo, el método propuesto en [12] es costoso, desde un punto de vista computacional.

Aquí consideraremos el problema (4.1) donde los coeficientes $A(t), B(t)$ y $F(t)$ son funciones analíticas en $|t|<c$ con valores en $C^{r \times r}$, digamos

$$
A(t)=\sum_{n \geq 0} A_{n} t^{n}, \quad B(t)=\sum_{n \geq 0} B_{n} t^{n}, \quad F(t)=\sum_{n \geq 0} F_{n} t^{n}, \quad|t|<c .
$$

El propósito del presente capítulo es la construcción de soluciones analíticonuméricas del problema (4.1) con una aproximación prefijada en un dominio $|t| \leq A<c$, y la organización del capítulo es como sigue. En el segundo apartado demostramos la convergencia de la serie solución del problema (4.1) en $|t|<c$, bajo las hipótesis (4.2). En el tercer apartado se demuestran algunos lemas técnicos importantes que posteriormente usaremos en el análisis del error. En el cuarto apartado analizaremos la siguiente cuestión: cómo construir una solución analítico-numérica en forma de serie finita en $|t| \leq A$, cuyo error con respecto a la solución exacta en forma de serie infinita esté acotado superior y uniformemente para un error admisible prefijado $\epsilon>0$. Además se incluirá un procedimiento iterativo para la construcción de la mencionada solución aproximada.

En todo el capítulo, la norma $\|D\|$ de una matriz $D \in C^{r \times r}$, es la 2-norma de $D$, definida en $[21$, p.56]

$$
\|D\|=\sup _{x \neq 0} \frac{\|D x\|_{2}}{\|x\|_{2}}
$$

donde para un vector $y$ de $C^{r},\|y\|_{2}$ denota la norma euclídea usual de $y$. Si $x$ es un número real, denotaremos por $[x]$ su parte entera.

\subsection{La convergencia de la serie solución}

FUNC. ESPECIALES Y EC. DIF. MATRICIALES 
En este apartado buscaremos una solución $X(t)$ del problema (4.1) en forma de serie analítica

$$
X(t)=\sum_{n \geq 0} X_{n} t^{n}, \quad|t|<c,
$$

donde $X_{n}$ son matrices en $C^{r \times r}$ por determinar. Tomando derivadas formales en (4.3) se tiene

$$
X^{\prime}(t)=\sum_{n \geq 0}(n+1) X_{n+1} t^{n} .
$$

Supongamos por un momento que una solución en la forma (4.3) existe, entonces por el teorema de Mertens para el producto de series matriciales, y por (4.2) y (4.3) se deduce que

$$
\begin{aligned}
& A(t) X(t)=\left(\sum_{n \geq 0} A_{n} t^{n}\right)\left(\sum_{n \geq 0} X_{n} t^{n}\right)=\sum_{n \geq 0}\left(\sum_{k=0}^{n} A_{n-k} X_{k}\right) t^{n}, \\
& X(t) B(t)=\left(\sum_{n \geq 0} X_{n} t^{n}\right)\left(\sum_{n \geq 0} B_{n} t^{n}\right)=\sum_{n \geq 0}\left(\sum_{k=0}^{n} X_{n-k} B_{k}\right) t^{n} .
\end{aligned}
$$

Imponiendo que la $X(t)$ dada por (4.3) cumpla (4.1), y teniendo en cuenta (4.4)-(4.6), se deduce que

$$
\begin{gathered}
\sum_{n \geq 0}(n+1) X_{n+1} t^{n} \\
=\sum_{n \geq 0}\left(\sum_{k=0}^{n} A_{n-k} X_{k}\right) t^{n}+\sum_{n \geq 0}\left(\sum_{k=0}^{n} X_{n-k} B_{k}\right) t^{n}+\sum_{n \geq 0} F_{n} t^{n} \\
=\sum_{n \geq 0}\left\{F_{n}+\sum_{k=0}^{n}\left(A_{n-k} X_{k}+X_{n-k} B_{k}\right)\right\} t^{n}
\end{gathered}
$$

Igualando los coeficientes con la misma potencia de $t^{n}$ en (4.7) se deduce que

$$
(n+1) X_{n+1}=F_{n}+\sum_{k=0}^{n}\left(A_{n-k} X_{k}+X_{n-k} B_{k}\right), \quad n \geq 0, \quad X_{0}=C .
$$

FUNC. ESPECIALES Y EC. DIF. MATRICIALES 
Tomando normas en (4.8) se tiene

$$
(n+1)\left\|X_{n+1}\right\| \leq\left\|F_{n}\right\|+\sum_{k=0}^{n}\left(\left\|A_{n-k}\right\|\left\|X_{k}\right\|+\left\|X_{n-k}\right\|\left\|B_{k}\right\|\right) .
$$

Por las desigualdades de Cauchy [14, p.222], existe una constante positiva $M>0$ tal que

$$
\left\|A_{n}\right\| \leq \frac{M}{\rho^{n}}, \quad\left\|B_{n}\right\| \leq \frac{M}{\rho^{n}}, \quad\left\|F_{n}\right\| \leq \frac{M}{\rho^{n}}, \quad 0<\rho<c, \quad n \geq 0 .
$$

Por (4.9) y (4.10) se deduce que

$$
(n+1)\left\|X_{n+1}\right\| \leq \frac{M}{\rho^{n}}\left(1+2 \sum_{k=0}^{n}\left\|X_{k}\right\| \rho^{k}\right), \quad n \geq 0, \quad 0<\rho<c .
$$

Luego

$$
\left\|X_{n+1}\right\| \leq \frac{M}{(n+1) \rho^{n}}\left(1+2 \sum_{k=0}^{n}\left\|X_{k}\right\| \rho^{k}\right), \quad n \geq 0, \quad 0<\rho<c .
$$

Definamos la sucesión de números reales positivos $\left\{\delta_{n}\right\}_{n \geq 0}$ definida por $\delta_{0}=\left\|X_{0}\right\|=\|C\|$, y $\delta_{n}$ para $n \geq 0$ como la solución de la ecuación

$$
\delta_{n+1}=\frac{M}{(n+1) \rho^{n}}\left(1+2 \sum_{k=0}^{n} \delta_{k} \rho^{k}\right), \quad n \geq 0 .
$$

Por la definición de $\left\{\delta_{n}\right\}_{n \geq 0} \mathrm{y}$ (4.12), usando el principio de inducción, es fácil probar que

$$
\left\|X_{n}\right\| \leq \delta_{n}, \quad n \geq 0
$$

Por (4.14), para probar la convergencia de la serie (4.3) donde $X_{n}$ está dado por (4.8), es suficiente demostrar la convergencia de la serie numérica

$$
\sum_{n \geq 0} \delta_{n} t^{n}, \quad|t|<c
$$

Por la definición de $\delta_{n}$, ver (4.13), se tiene

$$
(n+1) \delta_{n+1}-\rho^{-1} n \delta_{n}=2 M \delta_{n}, \quad n>0 .
$$


Luego

$$
\frac{\delta_{n+1}}{\delta_{n}}=\frac{2 M \rho+n}{(n+1) \rho}
$$

y en consecuencia

$$
\lim _{n \rightarrow \infty} \frac{\delta_{n+1}|t|^{n+1}}{\delta_{n}|t|^{n}}=\frac{|t|}{\rho}<1, \quad \text { si } \quad|t|<\rho
$$

Luego (4.15) converge si $|t|<\rho$, donde $\rho$ es cualquier número positivo cumpliendo: $0<\rho<c$, i.e, la serie (4.15) converge en $|t|<c$. Esto significa que la serie (4.3), (4.8), no es sólo una solución formal, sino también la solución rigurosa del problema (4.1).

Nota 1. Dado un punto $t_{0}$ con $0<\left|t_{0}\right|<c$, por las propiedades de las funciones analíticas, las funciones $A(t), B(t)$ y $F(t)$ admiten un desarrollo en serie de potencias de la forma

$$
\begin{aligned}
& A(t)=\sum_{n \geq 0} A_{n}\left(t_{0}\right)\left(t-t_{0}\right)^{n}, \quad\left|t-t_{0}\right|<c-\left|t_{0}\right| \\
& B(t)=\sum_{n \geq 0} B_{n}\left(t_{0}\right)\left(t-t_{0}\right)^{n}, \quad\left|t-t_{0}\right|<c-\left|t_{0}\right| \\
& F(t)=\sum_{n \geq 0} F_{n}\left(t_{0}\right)\left(t-t_{0}\right)^{n}, \quad\left|t-t_{0}\right|<c-\left|t_{0}\right|
\end{aligned}
$$

Si consideramos el problema de valor inicial

$$
X^{\prime}(t)=A(t) X(t)+X(t) B(t)+F(t) ; \quad X\left(t_{0}\right)=C\left(t_{0}\right), \quad t_{0} \leq t<c,
$$

por los argumentos previos es sencillo comprobar que la solución exacta en forma de serie de potencias matricial del problema de valor inicial (4.17) está dada por

$$
\begin{gathered}
X(t)=\sum_{n \geq 0} X_{n}\left(t_{0}\right)\left(t-t_{0}\right)^{n}, \quad t_{0} \leq t<c, \\
X_{0}\left(t_{0}\right)=C\left(t_{0}\right), \\
X_{n+1}=\frac{1}{n+1}\left\{F_{n}\left(t_{0}\right)+\sum_{k=0}^{n}\left(A_{n-k}\left(t_{0}\right) X_{k}\left(t_{0}\right)+X_{n-k}\left(t_{0}\right) B_{k}\left(t_{0}\right)\right)\right\}, \quad n \geq 0
\end{gathered}
$$

FUNC. ESPECIALES Y EC. DIF. MATRICIALES 
Además, por las desigualdades de Cauchy [14, p.222] aplicadas en el disco $\left|t-t_{0}\right|<c-\left|t_{0}\right|$, se tiene

$$
\begin{aligned}
& \left\|A_{n}\left(t_{0}\right)\right\| \leq \frac{M}{\left(c-\left|t_{0}\right|\right)^{n}}, \quad n \geq 0 \\
& \left\|B_{n}\left(t_{0}\right)\right\| \leq \frac{M}{\left(c-\left|t_{0}\right|\right)^{n}}, \quad n \geq 0 \\
& \left\|F_{n}\left(t_{0}\right)\right\| \leq \frac{M}{\left(c-\left|t_{0}\right|\right)^{n}}, \quad n \geq 0
\end{aligned}
$$

donde $M \geq \sup \left\{\|A(t)\|,\|B(t)\|,\|F(t)\| \quad ; \quad\left|t-t_{0}\right| \leq c-t_{0}\right\}$.

Para mayor claridad en la notación, en lo sucesivo los coeficientes de los desarrollos en serie de potencias de $A(t), B(t)$, y $F(t)$ alrededor del punto $t=(j-1) b_{1}, j>1$, se denotarán por $A_{n}(j-1), B_{n}(j-1)$ y $F_{n}(j-1)$ respectivamente.

\subsection{Lemas técnicos}

Empezamos esta sección con un resultado que proporciona una cota de error a priori de la solución teórica del problema (4.1), y que utilizaremos en el siguiente apartado, cuando estudiemos el error de la solución aproximada que construiremos.

Lema 4.3.1 . Sean $A(t), B(t)$ y $F(t)$ funciones continuas, definidas sobre $[0, A]$ y con valores en $C^{r \times r}$, y sea $X(t)$ la solución del problema (4.1) en $[0, A]$. Entonces, para $0 \leq t \leq A$ se cumple

$$
\|X(t)\| \leq\left(\|C\|+\int_{0}^{A}\|F(s)\| d s\right) \exp \left(\int_{0}^{A}(\|A(s)\|+\|B(s)\|) d s\right),
$$

Demostración. Integrando la ecuación diferencial (4.1) se tiene que la solución $X(t)$ verifica

$$
X(t)-C=\int_{0}^{t}\{A(s) X(s)+X(s) B(s)+F(s)\} d s
$$

FUNC. ESPECIALES Y EC. DIF. MATRICIALES 
Sea $f(t)=\|X(t)\|$ y $g(t)=\|A(t)\|+\|B(t)\|$. Tomando normas en (4.19) se deduce que

$$
f(t) \leq f(0)+\int_{0}^{t} g(s) f(s) d s, \quad 0 \leq t \leq A
$$

Aplicando la desigualdad de Gronwall [18, p.95] a (4.20), se tiene (4.18).

Lema 4.3.2 . Sean $A(t), B(t)$ y $F(t)$ funciones continuas con valores en $C^{r \times r}$, sea $X_{1}(t)$ la solución de

$$
X_{1}^{\prime}(t)=A(t) X_{1}(t)+X_{1}(t) B(t)+F(t), \quad X_{1}(\alpha)=P, \quad \alpha \leq t \leq \beta,
$$

y sea $X_{2}(t)$ la solución de

$$
X_{2}^{\prime}(t)=A(t) X_{2}(t)+X_{2}(t) B(t)+F(t), \quad X_{2}(\alpha)=Q, \quad \alpha \leq t \leq \beta,
$$

Entonces, para $\alpha \leq t \leq \beta$ se cumple

$$
\left\|X_{1}(t)-X_{2}(t)\right\| \leq\|P-Q\| \exp \{(\beta-\alpha)(\|A(t)\|+\|B(t)\|)\},
$$

Demostración. A partir de las soluciones $X_{1}(t)$ y $X_{2}(t)$, definamos la función $G(t)=X_{1}(t)-X_{2}(t)$. Integrando (4.21) y (4.22) se tiene

$$
\begin{aligned}
& X_{1}(t)=P+\int_{\alpha}^{t}\left\{A(s) X_{1}(s)+X_{1}(s) B(s)+F(s)\right\} d s, \quad \alpha \leq t \leq \beta, \\
& X_{2}(t)=Q+\int_{\alpha}^{t}\left\{A(s) X_{2}(s)+X_{2}(s) B(s)+F(s)\right\} d s, \quad \alpha \leq t \leq \beta,
\end{aligned}
$$

Luego

$$
G(t)=P-Q+\int_{\alpha}^{t}\left\{A(s)\left(X_{1}(s)-X_{2}(s)\right)+\left(X_{1}(s)-X_{2}(s)\right) B(s)\right\} d s
$$

Tomando normas en (4.24) y por denotando $g(t)=\|G(t)\|$, se deduce que

$$
g(t) \leq\|P-Q\|+\int_{\alpha}^{t}(\|A(s)\|+\|B(s)\|) g(s) d s, \quad \alpha \leq t \leq \beta .
$$

Finalmente, aplicando la desigualdad de Gronwall a (4.25) ver [18, p.95], obtenemos (4.23).

Para mayor claridad en la presentación de los resultados siguientes, recordamos el siguiente lema relativo a la sumación de series dobles, cuya demostración puede encontrarse en [55, p.173].

FUNC. ESPECIALES Y EC. DIF. MATRICIALES 
Lema 4.3.3 . Dada una sucesión doble $\left\{a_{i j}\right\}, i \geq 1, j \geq 1$, supongamos que

$$
\sum_{j \geq 1}\left|a_{i j}\right|=b_{i}, \quad i \geq 1,
$$

y que $\sum_{i \geq 1} b_{i}$ converge. Entonces

$$
\sum_{i \geq 1} \sum_{j \geq 1} a_{i j}=\sum_{j \geq 1} \sum_{i \geq 1} a_{i j}
$$

\subsection{Construcción de aproximaciones precisas}

En esta sección, bajo las hipótesis (4.2) analizamos la siguiente cuestión: dado un dominio acotado $[0, A]$, con $A<c$, y un error admisible $\epsilon>0$, "cómo construir una solución aproximada finita $X(t, \epsilon)$ definida en $[0, A]$ de manera que dicha solución esté, con respecto a la solución en forma de serie infinita dada en el segundo apartado, acotada superior y uniformemente por $\epsilon$ en $[0, A]$ ?.

Dado $\epsilon>0$ y $A>1$, sea $h=[A]+1$ y denotemos por

$$
b_{1}=\frac{A}{[A]+1}<1, \quad b_{1} h=A .
$$

Sean $b$ y $a$ números positivos tales que

$$
0<b_{1}<b<1, \quad A<a<c, \quad b<b_{1}+(a-A),
$$

donde $b_{1}$ está definido por (4.28) y $c$ por (4.2). Notemos que de esta forma el intervalo $[0, A]$ está dividido en $h$ subintervalos

$$
\left[0, b_{1}\right],\left[b_{1}, 2 b_{1}\right], \ldots,\left[(h-1) b_{1}, A\right] .
$$

Por lo desarrollado en la segunda sección, sabemos que la solución exacta del problema (4.1) en $\left[0, b_{1}\right]$ está dada por

$$
\begin{gathered}
X_{1}(t)=\sum_{n \geq 0} X_{n} t^{n}, \quad 0 \leq t \leq b_{1} \\
X_{0}=C, \quad X_{n+1}=\frac{1}{n+1}\left\{F_{n}+\sum_{k=0}^{n}\left(A_{n-k} X_{k}+X_{n-k} B_{k}\right)\right\} \quad n \geq 0,
\end{gathered}
$$

FUNC. ESPECIALES Y EC. DIF. MATRICIALES 
Ahora consideramos la serie truncada de orden $m$ de $X_{1}(t)$

$$
Y_{1}(t, m)=\sum_{n=0}^{m} X_{n} t^{n}, \quad 0 \leq t \leq b_{1} .
$$

Para $|t| \leq b_{1}$ se deduce que

$$
\left\|X_{1}(t)-Y_{1}(t, m)\right\|=\left\|\sum_{n \geq m+1} X_{n} t^{n}\right\| \leq \sum_{n \geq m+1}\left\|X_{n}\right\| b_{1}^{n}
$$

Sea $\left\|X_{n}\right\|=\varphi_{n}$, y sea $M>0$ de manera que

$$
\sup _{0 \leq t \leq a}\{\|A(t)\|,\|B(t)\|,\|F(t)\|,\|X(t)\|\} \leq M,
$$

y recordemos por el lema 4.3.1 anterior que un tal valor de $M$ es fácil de obtener en términos de los datos. Por las desigualdades de Cauchy y el segundo apartado se tiene (ver 4.12)

$$
\begin{aligned}
& \varphi_{n+1} \leq \frac{M}{(n+1) b^{n}}\left(1+2 \sum_{k=0}^{n} \varphi_{k} b^{k}\right), \quad n \geq 0 \\
& \varphi_{n} \leq \frac{M}{n b^{n-1}}\left(1+2 \sum_{k=0}^{n-1} \varphi_{k} b^{k}\right), \quad n \geq 1,
\end{aligned}
$$

Por (4.32) y (4.34) se tiene

$$
\left.\begin{array}{c}
\left\|X_{1}(t)-Y_{1}(t, m)\right\| \\
\leq \sum_{n \geq m+1}\left\{\frac{M}{n b^{n-1}}\left(1+2 \sum_{k=0}^{n-1} \varphi_{k} b^{k}\right)\right\} b_{1}^{n} \\
=M b \sum_{n \geq m+1} \frac{1}{n}\left(\frac{b_{1}}{b}\right)^{n}+\sum_{n \geq m+1}\left(\frac{2 M}{n b^{n-1}} \sum_{k=0}^{n-1} \varphi_{k} b^{k}\right) b_{1}^{n} \\
\leq M b \sum_{n \geq m+1}\left(\frac{b_{1}}{b}\right)^{n}+\sum_{n \geq m+1}\left(\frac{2 M}{n b^{n-1}} \sum_{k=0}^{n-1} \varphi_{k} b^{k}\right) b_{1}^{n}
\end{array}\right]
$$

FUNC. ESPECIALES Y EC. DIF. MATRICIALES 
Por la convergencia de la serie $\sum_{n \geq m+1} \varphi_{n} b_{1}^{n}$ y el lema 4.3.3 de este capítulo, podemos escribir

$$
\begin{gathered}
\sum_{n \geq m+1}\left(\frac{2 M}{n b^{n-1}} \sum_{k=0}^{n-1} \varphi_{k} b^{k}\right) b_{1}^{n}=2 M b \varphi_{0} \sum_{j \geq 1} \frac{1}{m+j}\left(\frac{b_{1}}{b}\right)^{m+j} \\
+2 M b^{2} \varphi_{1} \sum_{j \geq 1} \frac{1}{m+j}\left(\frac{b_{1}}{b}\right)^{m+j}+2 M b^{3} \varphi_{2} \sum_{j \geq 1} \frac{1}{m+j}\left(\frac{b_{1}}{b}\right)^{m+j} \\
+2 M b^{m+1} \varphi_{m} \sum_{j \geq 1} \frac{1}{m+j}\left(\frac{b_{1}}{b}\right)^{m+j}+2 M b^{m+2} \varphi_{m+1} \sum_{j \geq 2} \frac{1}{m+j}\left(\frac{b_{1}}{b}\right)^{m+j} \\
+2 M b^{m+3} \varphi_{m+2} \sum_{j \geq 3} \frac{1}{m+j}\left(\frac{b_{1}}{b}\right)^{m+j} \cdots \\
+2 M b^{m+l} \varphi_{m+l-1} \sum_{j \geq l} \frac{1}{m+j}\left(\frac{b_{1}}{b}\right)^{m+j} \cdots
\end{gathered}
$$


Luego

$$
\begin{gathered}
\sum_{n \geq m+1}\left(\frac{2 M}{n b^{n-1}} \sum_{k=0}^{n-1} \varphi_{k} b^{k}\right) b_{1}^{n} \\
=\left\{2 M b \sum_{j \geq 1} \frac{1}{m+j}\left(\frac{b_{1}}{b}\right)^{m+j}\right\}\left(\varphi_{0}+b \varphi_{1}+\ldots+b^{m} \varphi_{m}\right) \\
+2 M b b^{m+1} \varphi_{m+1} \sum_{j \geq 2} \frac{1}{m+j}\left(\frac{b_{1}}{b}\right)^{m+j} \\
+2 M b b^{m+2} \varphi_{m+2} \sum_{j \geq 3} \frac{1}{m+j}\left(\frac{b_{1}}{b}\right)^{m+j} \\
+\ldots \\
+2 M b b^{m+l-1} \varphi_{m+l-1} \sum_{j \geq l} \frac{1}{m+j}\left(\frac{b_{1}}{b}\right)^{m+j}+\ldots
\end{gathered}
$$

Como para $l \geq 1$, se tiene

$$
\begin{gathered}
\sum_{j \geq l}\left(\frac{b_{1}}{b}\right)^{m+j}=\left(\frac{b_{1}}{b}\right)^{m} \sum_{j \geq l}\left(\frac{b_{1}}{b}\right)^{j}=\left(\frac{b_{1}}{b}\right)^{m} \frac{\left(b_{1} / b\right)^{l}}{1-\frac{b_{1}}{b}} \\
\leq\left(\frac{b_{1}}{b}\right)^{m} \frac{\frac{b_{1}}{b}}{1-\frac{b_{1}}{b}}=\frac{b_{1}}{b-b_{1}}\left(\frac{b_{1}}{b}\right)^{m}, \\
\sum_{j \geq l} \frac{1}{m+j}\left(\frac{b_{1}}{b}\right)^{m+j} \leq \sum_{j \geq l}\left(\frac{b_{1}}{b}\right)^{m+j},
\end{gathered}
$$

FUNC. ESPECIALES Y EC. DIF. MATRICIALES 
por (4.36), se deduce que

$$
\begin{gathered}
\sum_{n \geq m+1}\left(\frac{2 M}{n b^{n-1}} \sum_{k=0}^{n-1} \varphi_{k} b^{k}\right) b_{1}^{n} \\
\leq \frac{2 M b\left(\frac{b_{1}}{b}\right)^{m}}{1-\frac{b_{1}}{b}}\left[\sum_{n=0}^{m} b^{n} \varphi_{n}+\sum_{n \geq m+1} \varphi_{n} b^{n}\left(\frac{b_{1}}{b}\right)^{n-m+1}\right] \\
\leq \frac{2 M b\left(\frac{b_{1}}{b}\right)^{m}}{1-\frac{b_{1}}{b}} \sum_{n \geq 0} b^{n} \varphi_{n} .
\end{gathered}
$$

Por las desigualdades de Cauchy [14, ,p.222], se sigue que

$$
\varphi_{n} \leq \frac{M}{a^{n}}, \quad n \geq 0,
$$

donde $M$ está dada por (4.33). Como

$$
\sum_{n \geq m+1}\left(\frac{b_{1}}{b}\right)^{n}=\frac{\left(b_{1} / b\right)^{m+1}}{1-\frac{b_{1}}{b}}
$$

por (4.35), (4.37), (4.38) y (4.39) se sigue que

$$
\begin{gathered}
\left\|X_{1}(t)-Y_{1}(t, m)\right\| \leq \frac{M b}{1-\frac{b_{1}}{b}}\left\{\left(b_{1} / b\right)^{m+1}+\frac{2 M\left(b_{1} / b\right)^{m}}{1-\frac{b}{a}}\right\} \\
\leq \frac{M b}{1-\frac{b_{1}}{b}}\left(1+\frac{2 M}{1-\frac{b}{a}}\right)\left(\frac{b_{1}}{b}\right)^{m}
\end{gathered}
$$

Supongamos por un momento que elegimos el primer entero positivo $m_{1}$ 
tal que

$$
\left(\frac{b_{1}}{b}\right)^{m_{1}}<\frac{\varepsilon\left(1-\frac{b_{1}}{b}\right)}{M b\left(1+\frac{2 M}{1-\frac{b}{a}}\right)\left[h+(h+1) e^{L b_{1}}\right]}
$$

donde

$$
L=\operatorname{máx}\{\|A(s)\|+\|B(s)\| ; \quad 0 \leq s \leq A\},
$$

Entonces por (4.40)-(4.41), se obtiene

$$
\left\|X_{1}(t)-Y_{1}\left(t, m_{1}\right)\right\| \leq \frac{\varepsilon}{h+(h-1) e^{L b_{1}}}, \quad|t| \leq b_{1}
$$

Observar que $m_{1}$ puede calcularse tomando el primer entero positivo $m_{1}$ verificando

$$
\ln \left\{\frac{\varepsilon\left(1-\frac{b_{1}}{b}\right)}{M b\left(1+\frac{2 M}{1-\frac{b}{a}}\right)\left[h+(h-1) e^{\left.L b_{1}\right]}\right.}\right\}
$$

Ahora consideramos el problema de valor inicial en $\left[b_{1}, 2 b_{1}\right]$

$$
X^{\prime}(t)=A(t) X(t)+X(t) B(t)+F(t) ; \quad X\left(b_{1}\right)=Y_{1}\left(b_{1}, m_{1}\right)
$$

Aplicando el método desarrollado en el segundo apartado y teniendo en cuenta la nota 1, la solución de (4.45) puede escribirse en la forma

$$
\begin{gathered}
X_{2}(t)=\sum_{n \geq 0} X_{n}(1)\left(t-b_{1}\right)^{n}, \quad b_{1} \leq t \leq 2 b_{1}, \\
X_{0}(1)=Y_{1}\left(b_{1}, m_{1}\right), \\
X_{n+1}(1)=\frac{1}{n+1}\left\{F_{n}(1)+\sum_{k=0}^{n}\left(A_{n-k}(1) X_{k}(1)+X_{n-k}(1) B_{k}(1)\right)\right\}
\end{gathered}
$$

FUNC. ESPECIALES Y EC. DIF. MATRICIALES 
donde $F_{n}(1), B_{n}(1)$ y $A_{n}(1)$ son los coeficientes del desarrollo en serie de potencias de Taylor de $F(t), B(t)$ y $A(t)$ respectivamente, alrededor del punto $t=b_{1}$. Notemos que por (4.33), y las desigualdades de Cauchy aplicadas a $F(t), B(t)$ y $A(t)$ en el disco $\left|t-b_{1}\right|<a-b_{1}=a_{1}$, se deduce que

$$
\left\|A_{n}(1)\right\| \leq \frac{M}{a_{1}^{n}}, \quad\left\|F_{n}(1)\right\| \leq \frac{M}{a_{1}^{n}}, \quad\left\|B_{n}(1)\right\| \leq \frac{M}{a_{1}^{n}}, \quad n \geq 0
$$

Si truncamos la serie (4.46) por su $m$-ésima suma parcial

$$
Y_{2}(t, m)=\sum_{n=0}^{m} X_{n}(1)\left(t-b_{1}\right)^{n}, \quad b_{1} \leq t \leq 2 b_{1},
$$

entonces por la nota $1,(4.47)$ y (4.44), sustituyendo $a$ por $a_{1}=a-b_{1}$, si $m_{2}$ es el primer entero positivo cumpliendo

$$
\ln \left\{\frac{\varepsilon\left(1-\frac{b_{1}}{b}\right)}{M b\left(1+\frac{2 M}{1-\frac{b}{a_{1}}}\right)\left[h+(h-1) e^{\left.L b_{1}\right]}\right.}\right\}
$$

deducimos que

$$
\left\|X_{2}(t)-Y_{2}\left(t, m_{2}\right)\right\| \leq \frac{\varepsilon}{h+(h-1) e^{L b_{1}}}, \quad b_{1} \leq t \leq 2 t_{1},
$$

donde $L$ está dado por (4.42). Inductivamente, razonamos igual para pasar de $\left[b_{1}, 2 b_{1}\right]$ a $\left[2 b_{1}, 3 b_{1}\right]$ y así sucesivamente, si denotamos por $Y_{j-1}\left(t, m_{j-1}\right)$ la 
aproximación de

$$
\left.\begin{array}{c}
X_{j}(t)=\sum_{n \geq 0} X_{n}(j-1)\left(t-(j-1) b_{1}\right)^{n}, \quad(j-1) b_{1} \leq t \leq j b_{1}, \\
X_{0}(j-1)=Y_{j-1}\left((j-1) b_{1}, m_{j-1}\right), \\
X_{n+1}(j-1)=\frac{1}{n+1}\left\{F_{n}(j-1)\right\} \\
+\frac{1}{n+1}\left\{\sum_{k=0}^{n}\left(A_{n-k}(j-1) X_{k}(j-1)+X_{n-k}(j-1) B_{k}(j-1)\right)\right\}
\end{array}\right]
$$

donde $m_{j}$ es el primer entero positivo verificando

$$
\ln \left\{\frac{\varepsilon\left(1-\frac{b_{1}}{b}\right)}{M b\left(1+\frac{2 M}{1-\frac{b}{a_{j-1}}}\right)\left[h+(h-1) e^{L b_{1}}\right]} m_{j}^{\ln b_{1} / b}\right\}
$$

$\mathrm{y}$

$$
a_{j-1}=a-(j-1) b_{1}, \quad 1 \leq j \leq h,
$$

por los argumentos previos, la serie truncada de orden $m_{j}$ de $X_{j}(t)$, definida por

$$
Y_{j}\left(t, m_{j}\right)=\sum_{n=0}^{m_{j}} X_{n}(j-1)\left(t-(j-1) b_{1}\right)^{n}
$$

cumple

$$
\left\|X_{j}(t)-Y_{j}\left(t, m_{j}\right)\right\| \leq \frac{\varepsilon}{h+(h-1) e^{L b_{1}}}, \quad(j-1) b_{1} \leq t \leq j b_{1}
$$

Observemos que para seleccionar $m_{j}$, hemos usado que las matrices coeficientes $A_{n}(j-1), B_{n}(j-1), F_{n}(j-1)$ de los desarrollos en serie de potencias de 
$A(t), B(t)$ y $F(t)$ respectivamente, verifican

$$
\left\|A_{n}(j-1)\right\| \leq \frac{M}{a_{j-1}^{n}},\left\|B_{n}(j-1)\right\| \leq \frac{M}{a_{j-1}^{n}},\left\|F_{n}(j-1)\right\| \leq \frac{M}{a_{j-1}^{n}}, n \geq 0
$$

Luego la solución aproximada $X(t, \varepsilon)$ definida por

$$
X(t, \varepsilon)=Y_{j}\left(t, m_{j}\right), \quad(j-1) b_{1} \leq t \leq j b_{1}, \quad 1 \leq j \leq h,
$$

donde $Y_{1}\left(t, m_{1}\right)$ está dada por (4.30)-(4.31), con $m=m_{1}$, y para los subíndices $1 \leq j \leq h, Y_{j}\left(t, m_{j}\right)$ está definida por (4.54), siendo $m_{j}$ el primer entero positivo cumpliendo (4.52).

Observemos que en el intervalo $\left[0, b_{1}\right]$ el error de aproximación entre la solución exacta en forma de serie $X(t)$ dada por $(4.30)$ y $X(t, \varepsilon)$ definida por (4.31), es el error de truncamiento acotado en (4.43). Sin embargo, en cada subintervalo $\left[(j-1) b_{1}, j b_{1}\right]$, para $2 \leq j \leq h$ tenemos dos contribuciones para el error: una procedente de la consideración de una condición inicial aproximada en $(j-1) b_{1}$, y otra producida por el error de truncamiento cuando consideramos la suma parcial $m_{j}$-ésima en vez de la serie infinita. Por tanto, para cualquier $t \in\left[0, h b_{1}\right]=[0, A]$, por los comentarios previos y el lema 4.3.2 anterior, se tiene

$$
\left.\begin{array}{c}
\|X(t)-X(t, \varepsilon)\| \leq \frac{\varepsilon}{h+(h-1) e^{L b_{1}}} \\
+\sum_{j=1}^{h-1}\left[\frac{\varepsilon}{h+(h-1) e^{L b_{1}}}+\frac{\varepsilon e^{L b_{1}}}{h+(h-1) e^{L b_{1}}}\right] \\
=\frac{h \varepsilon}{h+(h-1) e^{L b_{1}}}+\frac{(h-1) \varepsilon e^{L b_{1}}}{h+(h-1) e^{L b_{1}}} \\
=\frac{\varepsilon}{h+(h-1) e^{L b_{1}}}\left[h+(h-1) e^{L b_{1}}\right]=\varepsilon
\end{array}\right]
$$

Notar que si $A<1$, entonces tomando $b_{1}=A$, la solución aproximada $X(t, \varepsilon)=Y_{1}(t, m)$ definida por la serie matricial finita (4.31), verifica además que

$$
\|X(t)-X(t, \varepsilon)\|<\varepsilon, \quad 0 \leq t \leq b_{1}=A
$$

FUNC. ESPECIALES Y EC. DIF. MATRICIALES 
Asímismo, notar que por la elección de $b$ dada en (4.29), incluso en el último subintervalo de este proceso constructivo, $\left[(h-1) b_{1}, h b_{1}=A\right]$, se verifica la siguiente desigualdad $a-(h-1) b_{1}=a_{h-1}=b_{1}+(a-A)>b$. En consecuencia la serie

$$
\sum_{n \geq 0}\left(\frac{b}{a_{h-1}}\right)^{n}<+\infty,
$$

y (4.40) se verifica sustituyendo $a$ por $a_{j-1}$, con $2 \leq j \leq h$.

Resumiendo podemos establecer el siguiente resultado, que responde a la pregunta planteada al principio de este apartado,

Teorema 4.4.1 . Consideremos el problema de valor inicial (4.1) en el intervalo $[0, A]$, donde $0<A<c$ y los coeficientes matriciales $A(t), B(t) y$ $F(t)$ son funciones analiticas en $|t|<c$ con valores en $C^{r \times r}$. Sea $X(t)$ la solución exacta en forma de serie de potencias dada por (4.3), (4.8).

Dado un error admisible $\varepsilon>0$, el siguiente procedimiento proporciona la construcción de una solución aproximada $X(t, \varepsilon)$, cuyo error con respecto a $X(t)$ está uniformemente acotado por $\varepsilon$ en el intervalo $[0, A]$ :

$$
\|X(t)-X(t, \varepsilon)\|<\varepsilon
$$

Caso 1. $A<1, \varepsilon>0$.

-Sea $b_{1}=A, h=1, b=1, a>1$. Calculamos $M$ cumpliendo (4.33) usando (4.18).

-Sea $m_{1}$ el primer entero positivo cumpliendo la desigualdad dada en $(4.44)$.

-Sean $X_{0}$ y $X_{n+1}$ para $0 \leq n \leq m_{1}-1$ definidas por (4.30).

-Entonces $X(t, \varepsilon)=Y_{1}(t, m)$ definida por (4.31) es la solución aproximada del problema (4.1) en $[0, A]$ verificando (4.59).

Caso 2. $A>1, \varepsilon>0$.

FUNC. ESPECIALES Y EC. DIF. MATRICIALES 
-Sea $h=[A]+1, b_{1}=\frac{A}{[A]+1}, A=b_{1} h, A<a<c, b<b_{1}+a-A$.

-Calculamos M cumpliendo (4.33) usando (4.18).

-Sea $m_{1}$ el primer entero positivo cumpliendo (4.44).

-Calculamos $A_{n}=A_{n}(0), B_{n}=B_{n}(0)$ y $F_{n}=F_{n}(0)$ siendo $n$ un número natural, tal que: $0 \leq n \leq m_{1}$, dadas por (4.2).

-Sea $Y_{1}\left(t, m_{1}\right)$ definido por (4.30)-(4.31).

- Sea $j=2$ y $a_{1}=a-b_{1}$.

-Sea $m_{2}$ el primer entero positivo verificando (4.49) e $Y_{2}\left(t, m_{2}\right)$ definido por (4.54) $\operatorname{con} j=2$ y $X_{n}(1)$ dado por (4.51).

-Inductivamente, para cada $j>2$, dado $Y_{j-1}\left(t, m_{j-1}\right)$ definida por (4.51)(4.54), para $j-1$ en vez de $j$, sea $m_{j}$ el primer entero positivo verificando (4.52), donde $a_{j-1}$ está definido por (4.53).

-Sea $Y_{j}\left(t, m_{j}\right)$ definido por (4.51)-(4.54) en $(j-1) b_{1} \leq t \leq j b_{1}$.

-Para $j=h$, construimos $Y_{h}\left(t, m_{h}\right)$ por (4.51)- (4.54), donde $m_{h}$ es el primer entero positivo verificando (4.52) con $a_{h-1}$ definido por (4.53).

-Entonces $X(t, \varepsilon)$ definido por (4.57) es la solución aproximada buscada del problema (4.1) en $[0, A]$ verificando (4.59). 


\section{Capítulo 5}

\section{SOBRE LA ECUACION DIFERENCIAL MATRICIAL NO SIMETRICA DE RICCATI CON COEFICIENTES ANALITICOS}

\subsection{Introducción}

Los sistemas diferenciales de Riccati para problemas de valores iniciales aparecen frecuentemente en importantes aplicaciones de la teoría de control clásica [41], [7], [53], [44], [20] y en técnicas de desacoplamiento tanto en el estudio numérico como analítico de problemas de contorno [2], [42].

Los sistemas de Riccati tienen además aplicaciones en la teoría del control no cooperativo apareciendo en economía o en problemas militares, ver [1], [3], [48] y [8], y las referencias que aparecen en ellos.

En este capítulo consideraremos ecuaciones diferenciales matriciales de Riccati no simétricas rectangulares del tipo

$$
W^{\prime}(t)=C(t)-D(t) W(t)-W(t) A(t)-W(t) B(t) W(t), \quad W(0)=W_{0},
$$


donde los coeficientes $C(t) \in C^{p \times q}, D(t) \in C^{p \times p}, A(t) \in C^{q \times q}, B(t) \in C^{q \times p}$ son funciones matriciales analíticas en el intervalo $0 \leq t \leq c$, y la función incógnita $W(t) \in C^{p \times q}$. El caso en que las dimensiones $p$ y $q$ de las matrices son distintas, i.e. cumplen que $p \neq q$, aparece por ejemplo cuando un sistema de ecuaciones diferenciales matriciales de Riccati de la forma

$$
\left.\begin{array}{c}
K_{1}^{\prime}(t)=-Q_{1}(t)-A^{T}(t) K_{1}(t)-K_{1}(t) A(t) \\
+K_{1}(t) S_{1}(t) K_{1}(t)+K_{1}(t) S_{2}(t) K_{2}(t), \\
\\
K_{2}^{\prime}(t)=-Q_{2}(t)-A^{T}(t) K_{2}(t)-K_{2}(t) A(t) \\
+K_{2}(t) S_{2}(t) K_{2}(t)+K_{2}(t) S_{1}(t) K_{1}(t), \\
K_{1}\left(t_{f}\right)=K_{1 f}, K_{2}\left(t_{f}\right)=K_{2 f}, \\
K_{i}(t), Q_{i}(t), A(t), S_{i}(t) \in C^{r \times r}, i=1,2
\end{array}\right]
$$

se escribe en forma compacta

$$
\begin{gathered}
K^{\prime}(t)=-Q(t)-P(t) K(t)-K(t) M(t)+K(t) N(t) K(t), \\
K=\left[\begin{array}{c}
K_{1} \\
K_{2}
\end{array}\right], Q=\left[\begin{array}{l}
Q_{1} \\
Q_{2}
\end{array}\right], P=\left[\begin{array}{cc}
A^{T} & 0 \\
0 & A^{T}
\end{array}\right], M=A, N=\left[\begin{array}{ll}
S_{1} & S_{2}
\end{array}\right] .
\end{gathered}
$$

Sistemas de Riccati del tipo (5.2) aparecen por ejemplo cuando se abordan problemas de control no cooperativos mediante estrategias de Nash, ver [11] y [59].

Para el caso en que las matrices coeficientes sean cuadradas, es decir, $p=q$, si $A(t)^{T}$ denota la matriz traspuesta de $A(t)$, la ecuación de Riccati (5.1) se denomina simétrica cuando los coeficientes de (5.1) son matrices reales y $D(t)=A(t)^{T}$.

A pesar que la ecuación de Riccati ha sido ya extensamente estudiada, sus soluciones numéricas cuentan con numerosos problemas tales como su integración a través de singularidades [57], o el estudio de cotas de error a priori en términos de los datos.

Lo que es más, esos estudios que han sido llevados a cabo, están casi exclusivamente referidos a casos autónomos, a pesar que muchos de los sistemas reales no son autónomos, [3], [8], [1] y [48]. Algunas excepciones pueden

FUNC. ESPECIALES Y EC. DIF. MATRICIALES 
encontrarse en [51], [43], [30], [35] y [57]. En [43] se propone un estudio basado en la integración directa de (5.1) usando rutinas bien conocidas y en un método fundamentado en una modificación del algoritmo de Davison-Maki. Los resultados numéricos se han comprobado sobre un conjunto concreto de ejemplos sin aportar información alguna sobre cotas de error en términos de los datos para el caso general. En [51] se reconstruye la solución de una ecuación de Riccati simétrica a partir de la determinación previa de los valores y vectores propios de la solución matricial.

El estudio de la ecuación de Riccati (5.1) está muy relacionado con el sistema lineal siguiente

$$
\left.\begin{array}{c}
X^{\prime}(t)=S(t) X(t), \quad X(0)=\left[\begin{array}{c}
I_{q} \\
W_{0}
\end{array}\right] ; \\
X(t)=\left[\begin{array}{c}
U(t) \\
V(t)
\end{array}\right], \quad S(t)=\left[\begin{array}{cc}
A(t) & B(t) \\
C(t) & -D(t)
\end{array}\right] \in C^{(p+q) \times(p+q)} .
\end{array}\right]
$$

El método propuesto en [30] está basado en la vectorización del problema (5.3) y utiliza el método de aproximaciones sucesivas. En [30], se dan cotas de error a priori, sin embargo el intervalo de existencia de la solución límite es muy pequeño, ya que, la aplicación del método de aproximaciones sucesivas no tiene en cuenta la estructura de la ecuación de Riccati. Aparte de este inconveniente, el método propuesto en [30] converge lentamente y es caro desde el punto de vista computacional. En [13], se propone un interesante método de integración numérica para la ecuación de Riccati no simétrica y no autónoma. Aunque se dan cotas de error para las soluciones numéricas, éstas están expresadas en términos de las propiedades geométricas y conllevan constantes dicotómicas las cuales en la práctica no son conocidas a priori. En [35] se explota la estructura matricial de la ecuación (5.1) y se utilizan métodos lineales de un paso para obtener una solución numérica discreta. Utilizando funciones $\beta$-splines lineales matriciales se construye una solución numérica continua a partir de la solución numérica discreta. El análisis del error permite la determinación del tamaño del paso del método discreto para conseguir un error admisible prefijado. El método utilizado en [35] es bastante general porque sólo requiere que los coeficientes matriciales sean dos veces continuamente diferenciables. Sin embargo, este método es computacionalmente caro porque el valor requerido del tamaño del paso suele ser muy

FUNC. ESPECIALES Y EC. DIF. MATRICIALES 
pequeño. Aparte de esto, la construcción de la solución numérica continua interpolando valores numéricos discretos conlleva una considerable cantidad de cálculos.

En [57] se dan una clase de métodos matriciales explícitos de un paso para las soluciones numéricas del problema (5.1). Tales métodos los cuales pueden observarse a continuación el método de Davison-Maki de Kenney, explotan la estructura de grupo matricial subyacente en la ecuación, además son sencillos y económicos desde el punto de vista de su implementación.

La solución analítico-numérica propuesta aquí, es la la solución exacta de un problema de Riccati con coeficientes polinomiales matriciales por truncación del desarrollo en serie de Taylor de los coeficientes de (5.1).

La organización de este capítulo es la siguiente. El segundo apartado trata de la construcción de la solución $X(t)$ en forma de serie del problema (5.3) suponiendo que los coeficientes de (5.1) son funciones analíticas a valores matriciales,

$$
S(t)=\sum_{n \geq 0} S_{n} t^{n}, \quad|t|<c .
$$

Si denotamos por $X(t, n)$ la serie truncada de orden $n$, como la solución $W(t)$ de (5.1) puede escribirse en la forma

$$
W(t)=\left\{\left[\begin{array}{ll}
0 & I_{p}
\end{array}\right] X(t)\right\}\left\{\left[\begin{array}{ll}
I_{q} & 0
\end{array}\right] X(t)\right\}^{-1}=V(t)[U(t)]^{-1}
$$

en el intervalo donde $U(t)$ es invertible, una aproximación razonable de $W(t)$ es

$$
W_{n}(t)=\left\{\left[\begin{array}{ll}
0 & I_{p}
\end{array}\right] X(t, n)\right\}\left\{\left[\begin{array}{ll}
I_{q} & 0
\end{array}\right] X(t, n)\right\}^{-1}
$$

en el intervalo donde $U_{n}(t)=\left[\begin{array}{ll}I_{q} & 0\end{array}\right] X(t, n)$ es invertible. En este mismo apartado, el segundo, determinamos también el orden de truncación y el intervalo donde $U_{n}(t)$ es invertible, en términos del error de aproximación $X(t)-X(t, n)$. En el apartado segundo se obtiene además una cota de error a priori de la solución $W(t)$ de (5.1). En el tercer apartado se lleva a cabo el análisis del error respondiendo a la siguiente pregunta: $\operatorname{si} \delta>0$ es la longitud del intervalo $[0, \delta]$ donde $W(t)$ es la solución de (5.1), y si $\gamma<\delta$, "cómo determinar el menor valor $n_{1}$ tal que $U_{n}(t)$ es invertible y $\left\|W(t)-W_{n}(t)\right\|<\varepsilon$

FUNC. ESPECIALES Y EC. DIF. MATRICIALES 
para $0 \leq t \leq \gamma, n \geq n_{1}$ ?. En la última sección, la cuarta, se estudia la estabilidad del método propuesto.

Si $C$ es una matriz en $C^{r \times q}$, denotaremos por $\|C\|$ su 2-norma, definida como la raíz cuadrada del valor propio máximo de $C^{H} C$ donde $C^{H}$ es la traspuesta conjugada de $C$, ver [24, p.295-312]. La 2-norma de una matriz puede ser eficientemente calculada con los paquetes Matlab y Mathematica. La matriz identidad en $C^{r \times r}$ la denotaremos por $I_{r}$. Si $A$ es una matriz en $C^{p \times q}$, y $B=\left[\begin{array}{c}I_{q} \\ A\end{array}\right]$ entonces $B^{H} B=I+A^{H} A$ y $\|B\|=\left(1+\|A\|^{2}\right)^{\frac{1}{2}}$. Concluimos la introducción recordando un resultado relativo a la permutación del orden de sumación de una serie doble cuya demostración puede encontrarse en [55, p.173]. Si $\left\{a_{i j}\right\}_{i \geq 1, j \geq 1}$, es una sucesión doble de números complejos tal que

$$
\sum_{j \geq 1}\left|a_{i j}\right|=b_{i}, \quad \sum_{i \geq 1} b_{i}<\infty,
$$

entonces

$$
\sum_{i \geq 1} \sum_{j \geq 1} a_{i j}=\sum_{j \geq 1} \sum_{i \geq 1} a_{i j}
$$

\subsection{Lemas técnicos}

Es bien conocida la conexión entre un sistema de Riccati y un sistema lineal cuya matriz de coeficientes involucra los coeficientes de la ecuación de Riccati. En el contexto numérico esta conexión ha sido notablemnte perseguida recientemente en [57] explotando la estructura de grupo matricial subyacente en la ecuación. Existen varias aproximaciones a la solución de sistemas lineales con coeficientes variables las cuales engloban propiedades cualitativas de la solución exacta. Algunas de las más relevantes son el método de los conmutadores iterados [27], y la visión del grupo de Lie estudiado en [26]. Estos métodos son además interesantes para construir buenas aproximaciones numéricas de problemas diferenciales no lineales como puede verse en [64].

En este apartado proponemos una solución aproximada en forma de serie de sistemas diferenciales matriciales lineales con coeficientes analíticos

FUNC. ESPECIALES Y EC. DIF. MATRICIALES 
usando la aproximación de Taylor. Aunque esta aproximación impone fuertes condiciones sobre los coeficientes y parece que no recupera tan bien las características analíticas de la solución exacta como lo hacen los métodos desarrollados en [26] y [27], mencionamos algunos de los beneficios posibles del método de desarrollo de Taylor. Primero, no es necesario calcular la solución fundamental del problema correspondiente. Segundo, como se demuestra posteriormente, las desigualdades de Cauchy son una excelente herramienta que proporciona cotas de error a priori de la serie truncada de aproximación. En tercer lugar, cuando el coeficiente del sistema es un polinomio matricial, entonces la solución del sistema es también un polinomio matricial. Este hecho permitirá recuperar algunas de las ventajas de los métodos simétricos propuestos en [57] para la solución numérica de problemas de Riccati porque la ecuación de Riccati perturbada después del truncamiento del desarrollo en forma de serie de Taylor de los coeficientes, es además una ecuación de Riccati con coeficientes polinomiales.

Buscamos una solución en forma de serie analítica del problema (5.3) del tipo

$$
X(t)=\sum_{n \geq 0} X_{n} t^{n}, \quad|t|<c,
$$

donde los coeficientes $X_{n}$ son matrices en $C^{(p+q) \times q}$ que están por determinar. Tomando derivadas formales en (5.6) se tiene

$$
X^{\prime}(t)=\sum_{n \geq 0}(n+1) X_{n+1} t^{n},
$$

y suponiendo por el momento que existe una solución de la forma (5.6), por el teorema de Mertens para el producto de series matriciales, por (5.6) y (5.7) se deduce que

$$
S(t) X(t)=\left(\sum_{n \geq 0} S_{n} t^{n}\right)\left(\sum_{n \geq 0} X_{n} t^{n}\right)=\sum_{n \geq 0}\left(\sum_{k=0}^{n} S_{n-k} X_{k}\right) t^{n}
$$

Imponiendo que $X(t)$ dada por (5.6) satisfaga (5.3) y teniendo en cuenta (5.7) se tiene que

$$
\sum_{n \geq 0}(n+1) X_{n+1} t^{n}=\sum_{n \geq 0}\left(\sum_{k=0}^{n} S_{n-k} X_{k}\right) t^{n} .
$$

FUNC. ESPECIALES Y EC. DIF. MATRICIALES 
Igualando los coeficientes de $t^{n}$ en (5.9) se sigue que

$$
(n+1) X_{n+1}=\sum_{k=0}^{n} S_{n-k} X_{k}, \quad n \geq 0, \quad X_{0}=\left[\begin{array}{c}
I_{q} \\
W_{0}
\end{array}\right] \in C^{(p+q) \times q}
$$

Tomando normas en (5.10) se tiene

$$
(n+1)\left\|X_{n+1}\right\| \leq \sum_{k=0}^{n}\left\|S_{n-k}\right\|\left\|X_{k}\right\|
$$

Por las desigualdades de Cauchy [14, p.222], tomando $M=\sup _{0 \leq t \leq c}\|S(t)\|$, se sigue que

$$
\left\|S_{n}\right\| \leq \frac{M}{\rho^{n}}, \quad 0<\rho<c, \quad n \geq 0 .
$$

Por (5.11) y (5.12) se deduce que

$$
\begin{gathered}
(n+1)\left\|X_{n+1}\right\| \leq \frac{M}{\rho^{n}} \sum_{k=0}^{n}\left\|X_{k}\right\| \rho^{k}, \quad n \geq 0, \quad 0<\rho<c . \\
\left\|X_{n+1}\right\| \leq \frac{M}{(n+1) \rho^{n}} \sum_{k=0}^{n}\left\|X_{k}\right\| \rho^{k}, \quad n \geq 0 .
\end{gathered}
$$

Introducimos ahora la sucesión de números positivos $\left\{\delta_{n}\right\}_{n \geq 0}$ definida por $\delta_{0}=\left\|X_{0}\right\|=\left(1+\left\|W_{0}\right\|^{2}\right)^{\frac{1}{2}}$, siendo $\delta_{n}$ para $n>0$ la solución de la ecuación

$$
\delta_{n+1}=\frac{M}{(n+1) \rho^{n}} \sum_{k=0}^{n} \delta_{k} \rho^{k}, \quad n \geq 0 .
$$

Por definición de $\left\{\delta_{n}\right\}_{n \geq 0}$ y (5.14), usando el principio de inducción, es fácil probar que

$$
\left\|X_{n}\right\| \leq \delta_{n}, \quad n \geq 0
$$

Por (5.16), para demostrar la convergencia de la serie (5.6) con $X_{n}$ dados por (5.10), es suficiente garantizar la convergencia de la serie numérica

$$
\sum_{n \geq 0} \delta_{n} t^{n}, \quad|t|<c
$$


Por la definición de $\delta_{n}$, ver (5.15), se tiene

$$
\begin{gathered}
(n+1) \delta_{n+1}-\rho^{-1} n \delta_{n}=M \delta_{n}, \quad n>0 . \\
\frac{\delta_{n+1}}{\delta_{n}}=\frac{\rho M+n}{(n+1) \rho}, \quad n>0, \\
\lim _{n \rightarrow \infty} \frac{\delta_{n+1}|t|^{n+1}}{\delta_{n}|t|^{n}}=\frac{|t|}{\rho}<1 \text { si } \quad|t|<\rho .
\end{gathered}
$$

Luego (5.6) converge en $|t|<\rho$, donde $\rho$ es cualquier número positivo con $0<\rho<c$. En consecuencia la serie (5.6), (5.10) es una solución rigurosa del problema (5.3). Por la unicidad de solución de (5.3), ver [18], la serie (5.6), (5.10) define la única solución del problema (5.3).

Sean $U(t)$ y $V(t)$ definidas por

$$
\begin{aligned}
& U(t)=\left[\begin{array}{ll}
I_{q} & 0
\end{array}\right] X(t) \\
& V(t)=\left[\begin{array}{ll}
0 & I_{p}
\end{array}\right] X(t)
\end{aligned}
$$

donde $X(t)$ es la solución del problema (5.3). Por [53, p.28], la solución $W(t)$ de (5.1) está definida en el intervalo donde $U(t)$ es invertible, y en ese dominio $W(t)$ está dada por

$$
W(t)=V(t)[U(t)]^{-1} .
$$

Recientemente, en el artículo [35] se ha establecido información sobre el intervalo de existencia de $W(t)$ en un resultado que a continuación enunciamos para mayor claridad en la presentación de las ideas.

La prueba del lema 5.2.1 está basada en el artículo [19] y es más fuerte que el lema 2.10 del trabajo [42] porque proporciona un método para obtener el intervalo de invertibilidad de $U(t)$ y no sólo su existencia.

Lema 5.2.1 . ([35]) Consideremos los números positivos $k_{0}, q_{0}$ y $\omega_{0}$, dados por

$$
\begin{gathered}
k_{0}=\operatorname{máx}\{\|S(t)\| ; 0 \leq t \leq c\}, \\
q_{0}=\operatorname{máx}\{\|[A(t) \quad B(t)]\| ; \quad 0 \leq t \leq c\},
\end{gathered}
$$

FUNC. ESPECIALES Y EC. DIF. MATRICIALES 


$$
\omega_{0}=\left\|\left[\begin{array}{c}
I_{q} \\
W_{0}
\end{array}\right]\right\|=\left(1+\left\|W_{0}\right\|^{2}\right)^{\frac{1}{2}}
$$

y sea $\delta$ un número positivo $\delta \leq c$, satisfaciendo

$$
\delta k_{0}+\ln (\delta)<-\ln \left(q_{0} \omega_{0}\right)
$$

Entonces la solución local $W(t)$ del problema (5.1) está dada por (5.18) en el intervalo $[0, \delta]$. Además, si $X(t)$ es la solución (5.3), entonces se verifica que $U(t)=\left[\begin{array}{ll}I_{q} & 0\end{array}\right] X(t)$ es invertible en $[0, \delta] y$

$$
\begin{gathered}
\left\|(U(t))^{-1}\right\|<(1-N \delta)^{-1}, \\
N=q_{0} \exp \left(\delta k_{0}\right) \omega_{0}, \quad 0 \leq t \leq \delta .
\end{gathered}
$$

Un importante resultado que necesitaremos utilizar más adelante es una cota superior a priori para la solución teórica $W(t)$ del problema (5.1), en el intervalo de existencia determinado en el lema 5.2.1 anterior.

Lema 5.2.2 . Bajo las hipótesis y la notación del lema 5.2.1 anterior, la solución $W(t)$ del problema (5.1) cumple

$$
\|W(t)\| \leq\left(1-\delta q_{0} \exp \left(\delta k_{0}\right) \omega_{0}\right)^{-1} \omega_{0} \exp \left(\delta k_{0}\right)=M_{2}, 0 \leq t \leq \delta .
$$

Demostración. Por [53, p.28] la solución $W(t)$ del problema (5.1) está dada por

$$
W(t)=V(t)[U(t)]^{-1}=\left[\begin{array}{ll}
0 & I_{p}
\end{array}\right] X(t)\left\{\left[\begin{array}{ll}
I_{q} & 0
\end{array}\right] X(t)\right\}^{-1}, \quad 0 \leq t \leq \delta .
$$

Por el lema 5.2.1 anterior se tiene

$$
\left\|[U(t)]^{-1}\right\| \leq\left(1-\delta q_{0} \exp \left(\delta k_{0}\right) \omega_{0}\right)^{-1}, \quad 0 \leq t \leq \delta .
$$

Además, por [18, p.114], la solución $X(t)$ del problema (5.3) verifica

$$
\|X(t)\| \leq \omega_{0} \exp \left(\delta k_{0}\right), \quad 0 \leq t \leq \delta .
$$

De aquí

$$
\|V(t)\| \leq\|X(t)\| \leq \omega_{0} \exp \left(\delta k_{0}\right), \quad 0 \leq t \leq \delta
$$

FUNC. ESPECIALES Y EC. DIF. MATRICIALES 
y por (5.22) se deduce (5.21). Por lo que el resultado queda probado.

La expresión (5.18) presenta dos dificultades desde un punto de vista computacional. En primer lugar, el hecho que $V(t)$ y $U(t)$ son series infinitas, y en segundo término, el cálculo de la inversa de $U(t)$. Pensando en el análisis del error de una aproximación $W_{n}(t)$ de $W(t)$ en términos de las aproximaciones $V_{n}(t)$ y $U_{n}(t)$ de $V(t)$ y $U(t)$, respectivamente, notemos que es conveniente garantizar la invertibilidad de $U_{n}(t)$ para poder escribir

$$
W_{n}(t)=V_{n}(t)\left[U_{n}(t)\right]^{-1},
$$

y

$$
W(t)-W_{n}(t)=V(t)[U(t)]^{-1}-V_{n}(t)\left[U_{n}(t)\right]^{-1} .
$$

El siguiente resultado responde a este hecho en términos de la calidad de las aproximaciones cuando truncamos la solución $X(t)$ en forma de serie infinita del problema (5.3).

Sea $0<\gamma<\delta$, donde $\delta$ está dado por el lema 5.2.1 anterior. Entonces con la notación del citado lema 5.2.1, ver expresión (2.27) de [35], se deduce que

$$
0<h(\gamma)=\gamma \exp \left(\gamma k_{0}\right)<\left[q_{0} \omega_{0}\right]^{-1},
$$

sea $\xi>0$ tal que

$$
h(\gamma)=\left[q_{0} \omega_{0}\right]^{-1}-\xi
$$

Sea

$$
X(t, n)=\sum_{k=0}^{n} X_{k} t^{k},
$$

la serie truncada de orden $n$ de $X(t)$, y sea

$$
U_{n}(t)=\left[\begin{array}{ll}
I_{q} & 0
\end{array}\right] X(t, n),
$$

el orden de truncación $n$ de $U(t)=\left[\begin{array}{ll}I_{q} & 0\end{array}\right] X(t)$. Notar que

$$
U_{n}(0)=I
$$

FUNC. ESPECIALES Y EC. DIF. MATRICIALES 
y

$$
\left.\begin{array}{c}
\left\|U_{n}(t)-I_{q}\right\| \leq\left\|U_{n}(t)-U(t)\right\|+\left\|U(t)-I_{q}\right\| \\
\leq\|X(t, n)-X(t)\|+\left\|U(t)-I_{q}\right\| .
\end{array}\right]
$$

Si $0 \leq t \leq \gamma$, entonces

$$
U^{\prime}(t)=\left[\begin{array}{ll}
I_{q} & 0
\end{array}\right] X^{\prime}(t)=\left[\begin{array}{ll}
I_{q} & 0
\end{array}\right] S(t) X(t)=\left[\begin{array}{ll}
A(t) & B(t)
\end{array}\right] X(t),
$$

y tomando normas en la última expresión, se deduce que

$$
\left\|U^{\prime}(t)\right\| \leq q_{0} \exp \left(\gamma k_{0}\right) \omega_{0}, \quad 0 \leq t \leq \gamma<\delta .
$$

Por (5.24), (5.25) y (5.29) se sigue que

$$
\gamma \sup _{0 \leq t \leq \gamma}\left\|U^{\prime}(t)\right\| \leq \gamma q_{0} \omega_{0} \exp \left(\gamma k_{0}\right)=1-\xi q_{0} \omega_{0}<1 .
$$

Por [18, p.255], se tiene que

$$
\left\|[U(t)]^{-1}\right\| \leq\left(\xi q_{0} \omega_{0}\right)^{-1}, \quad 0 \leq t \leq \gamma .
$$

Por (5.30) y por el teorema del valor medio [14, p.158], para $0 \leq t \leq \gamma$, se deduce que

$$
\left\|U(t)-I_{q}\right\|=\|U(t)-U(0)\| \leq \gamma \sup _{0 \leq s \leq \gamma}\left\|U^{\prime}(s)\right\| \leq 1-\xi q_{0} \omega_{0}, \quad 0 \leq t \leq \gamma
$$

Supongamos que

$$
\|X(t)-X(t, n)\|<\xi q_{0} \omega_{0}, \quad 0 \leq t \leq \gamma ; \quad n \geq n_{0}
$$

entonces por $(5.28),(5.32)$ y $(5.33)$ se tiene

$$
\left\|U_{n}(t)-I_{q}\right\|<1, \quad n \geq n_{0}, \quad 0 \leq t \leq \gamma
$$

y por el lema de perturbación [50, p.45], la matriz $U_{n}(t)$ es invertible en $[0, \gamma]$. Supongamos además que

$$
\left\|X^{\prime}(t, m)-X^{\prime}(t)\right\|<\delta^{-1} \xi q_{0} \omega_{0}, \quad n \geq n_{0}, \quad 0 \leq t \leq \gamma .
$$

Como

$$
U_{n}^{\prime}(t)-U^{\prime}(t)=\left[\begin{array}{ll}
I_{q} & 0
\end{array}\right]\left[X^{\prime}(t, n)-X^{\prime}(t)\right]
$$


podemos escribir

$$
\begin{gathered}
\left\|U_{n}^{\prime}(t)\right\| \leq\left\|U_{n}^{\prime}(t)-U^{\prime}(t)\right\|+\left\|U^{\prime}(t)\right\| \\
\quad \leq\left\|X^{\prime}(t, n)-X^{\prime}(t)\right\|+\left\|U^{\prime}(t)\right\| .
\end{gathered}
$$

Por (5.29) y (5.35) se deduce que

$$
\left\|U_{n}^{\prime}(t)\right\|<\left[\delta^{-1} \xi+\exp \left(\gamma k_{0}\right)\right] q_{0} \omega_{0}, \quad 0 \leq t \leq \gamma, \quad n \geq n_{0} .
$$

Por [19, p.255] se tiene

$$
\left\|\left(U_{n}(t)\right)^{-1}\right\| \leq\left\{1-\gamma\left[\delta^{-1} \xi+\exp \left(\gamma k_{0}\right)\right] q_{0} \omega_{0}\right\}^{-1}, n \geq n_{0}, \quad 0 \leq t \leq \gamma
$$

Por (5.24), (5.25) podemos escribir

$$
\begin{array}{r}
1-\gamma\left[\delta^{-1} \xi+\exp \left(\gamma k_{0}\right)\right] q_{0} \omega_{0} \\
=1-\gamma \delta^{-1} \xi q_{0} \omega_{0}-\gamma q_{0} \exp \left(\gamma k_{0}\right) \omega_{0} \\
=1-\gamma \delta^{-1} \xi q_{0} \omega_{0}-1+\xi q_{0} \omega_{0} \\
=\left(1-\gamma \delta^{-1}\right) \xi q_{0} \omega_{0} .
\end{array}
$$

Por (5.37) se sigue que

$$
\left\|\left(U_{n}(t)\right)^{-1}\right\| \leq\left\{\left(1-\gamma \delta^{-1}\right) \xi q_{0} \omega_{0}\right\}^{-1}, 0 \leq t \leq \gamma, \quad n \geq n_{0} .
$$

Resumiendo, podemos establecer el siguiente resultado:

Lema 5.2.3 . Con la notación del lema 5.2.1 anterior, sea $\gamma<\delta$ y $\xi>0$ de manera que

$$
\gamma \exp \left(\gamma k_{0}\right)=\left[q_{0} \omega_{0}\right]^{-1}-\xi,
$$

sea $X(t)$ la solución del problema (5.3), $U(t)=\left[\begin{array}{ll}I_{q} & 0\end{array}\right] X(t)$, sea $X(t, n)=$ $\sum_{k=0}^{n} X_{k} t^{k}$, la truncación de orden $n$ de $X(t)$, donde $S(t)$ es una función matricial analítica definida sobre el intervalo $[0, \delta]$ y con valores en $C^{(p+q) \times(p+q)}$. 
Entonces las siguientes propiedades se verifican:

(i) $U(t)$ es invertible $y$

$$
\left\|(U(t))^{-1}\right\|<\left(\xi q_{0} \omega_{0}\right)^{-1}, \quad 0 \leq t \leq \gamma .
$$

(ii) Si $n_{0}$ se elige de modo que

$$
\begin{aligned}
& \|X(t)-X(t, n)\|<\xi q_{0} \omega_{0}, \quad 0 \leq t \leq \gamma, \quad n \geq n_{0}, \\
& \left\|X^{\prime}(t)-X^{\prime}(t, n)\right\|<\delta^{-1} \xi q_{0} \omega_{0}, \quad 0 \leq t \leq \gamma, \quad n \geq n_{0}, \\
& \text { entonces } U_{n}(t) \text { es invertible en }[0, \delta] \text { para } n \geq n_{0}, y \\
& \left\|\left(U_{n}(t)\right)^{-1}\right\|<\left\{\left(1-\gamma \delta^{-1}\right) \xi q_{0} \omega_{0}\right\}^{-1}, \quad 0 \leq t \leq \gamma, \quad n \geq n_{0} .
\end{aligned}
$$

\subsection{Error de truncación}

En esta sección, bajo las hipótesis dadas en (5.4), lanzamos la siguiente cuestión: dado un error admisible $\epsilon>0$ y un dominio acotado $[0, \gamma] \operatorname{con} \gamma<\delta$, “cómo construir una solución aproximada finita $W(t, \epsilon)$ definida en $[0, \gamma]$ de modo que el error con respecto a la solución exacta $W(t)=V(t)[U(t)]^{-1}$ esté uniformemente acotado por $\epsilon$ en $[0, \gamma]$ ?

Sea $X(t)$ la solución en forma de serie infinita del problema (5.3). En primer lugar consideraremos el error de truncamiento de la aproximación de grado $m$ sobre un intervalo de longitud $\gamma<\delta$ :

$$
\begin{gathered}
X(t, m)=\sum_{n=0}^{m} X_{n} t^{n}, \quad 0 \leq t \leq \gamma<\delta, \\
X_{0}=\left[\begin{array}{c}
I_{q} \\
W_{0}
\end{array}\right] \\
X_{n+1}=\frac{1}{n+1} \sum_{k=0}^{n} S_{n-k} X_{k}, \quad n \geq 0 .
\end{gathered}
$$

FUNC. ESPECIALES Y EC. DIF. MATRICIALES 
Sea $M_{1}$ de manera que

$$
\sup _{0 \leq t \leq \delta}\|S(t)\| \leq M_{1}
$$

y aplicando el lema 5.2.2 anterior se deduce

$$
\sup _{0 \leq t \leq \delta}\|X(t)\| \leq M_{2}
$$

donde $M_{2}$ está dado por el miembro derecho de (5.21).

Por las desigualdades de Cauchy [14, p.222] en $\left[0, t_{1}\right]$, se obtiene que

$$
\left\|S_{n}\right\| \leq \frac{M_{1}}{t_{1}^{n}}, \quad 0<\gamma<t_{1}<\delta, \quad n \geq 0 .
$$

Por (5.40) y (5.43) los números $\varphi_{n}=\left\|X_{n}\right\|$ verifican

$$
\varphi_{n+1} \leq \frac{M_{1}}{(n+1) t_{1}^{n}} \sum_{k=0}^{n} \varphi_{k} t_{1}^{k}, \quad n \geq 0 .
$$

Luego

$$
X(t)-X(t, m)=\sum_{n \geq m+1} X_{n} t^{n}, \quad 0 \leq t \leq \gamma .
$$

luego el error de trncamiento cumple

$$
\|X(t)-X(t, m)\| \leq \sum_{n \geq m+1}\left\|X_{n}\right\| \gamma^{n} \leq \sum_{n \geq m+1} \frac{M_{1}}{n t_{1}^{n-1}}\left(\sum_{k=0}^{n-1} \varphi_{k} t_{1}^{k}\right) \gamma^{n} .
$$

Usando el resultado (5.5) relativo a la permutación del orden de sumación de una serie doble de números complejos y la convergencia de la serie de términos positivos

$$
\sum_{n \geq 1} \varphi_{n} \gamma^{n}
$$

FUNC. ESPECIALES Y EC. DIF. MATRICIALES 
podemos escribir

$$
\begin{gathered}
\sum_{n \geq m+1} \frac{1}{n t_{1}^{n-1}}\left(\sum_{k=0}^{n-1} \varphi_{k} t_{1}^{k}\right) \gamma^{n} \\
=t_{1} \varphi_{0} \sum_{j \geq 1} \frac{1}{m+j}\left(\frac{\gamma}{t_{1}}\right)^{m+j}+t_{1}^{2} \varphi_{1} \sum_{j \geq 1} \frac{1}{m+j}\left(\frac{\gamma}{t_{1}}\right)^{m+j}+\ldots \\
+t_{1}^{m+1} \varphi_{m} \sum_{j \geq 1} \frac{1}{m+j}\left(\frac{\gamma}{t_{1}}\right)^{m+j}+t_{1}^{m+2} \varphi_{m+1} \sum_{j \geq 2} \frac{1}{m+j}\left(\frac{\gamma}{t_{1}}\right)^{m+j} \\
+t_{1}^{m+3} \varphi_{m+2} \sum_{j \geq 3} \frac{1}{m+j}\left(\frac{\gamma}{t_{1}}\right)^{m+j}+\ldots+t_{1}^{m+l} \varphi_{m+l-1} \sum_{j \geq l} \frac{1}{m+j}\left(\frac{\gamma}{t_{1}}\right)^{m+j}+\ldots
\end{gathered}
$$

Luego

$$
\begin{gathered}
\sum_{n \geq m+1} \frac{1}{n t_{1}^{n-1}}\left(\sum_{k=0}^{n-1} \varphi_{k} t_{1}^{k}\right) \gamma^{n} \\
=\left\{t_{1} \sum_{j \geq 1} \frac{1}{m+j}\left(\frac{\gamma}{t_{1}}\right)^{m+j}\right\}\left(\varphi_{0}+t_{1} \varphi_{1}+\ldots+t_{1}^{m} \varphi_{m}\right) \\
+t_{1}\left\{t_{1}^{m+1} \varphi_{m+1} \sum_{j \geq 2} \frac{1}{m+j}\left(\frac{\gamma}{t_{1}}\right)^{m+j}+t_{1}^{m+2} \varphi_{m+2} \sum_{j \geq 3} \frac{1}{m+j}\left(\frac{\gamma}{t_{1}}\right)^{m+j}+\ldots+\right. \\
\left.+t_{1}^{m+l} \varphi_{m+l} \sum_{j \geq l+1} \frac{1}{m+j}\left(\frac{\gamma}{t_{1}}\right)^{m+j}+\ldots\right\} .
\end{gathered}
$$

Para $l \geq 1$, podemos escribir una cota superior común para todo natural $l$ de la serie geométrica de términos positivos

$$
\sum_{j \geq l}\left(\frac{\gamma}{t_{1}}\right)^{m+j}
$$

ya que, se tiene

FUNC. ESPECIALES Y EC. DIF. MATRICIALES 


$$
\begin{gathered}
\sum_{j \geq l}\left(\frac{\gamma}{t_{1}}\right)^{m+j}=\left(\frac{\gamma}{t_{1}}\right)^{m} \sum_{j \geq l}\left(\frac{\gamma}{t_{1}}\right)^{j}=\left(\frac{\gamma}{t_{1}}\right)^{m} \frac{\left(\frac{\gamma}{t_{1}}\right)^{l}}{1-\frac{\gamma}{t_{1}}} \\
\leq\left(\frac{\gamma}{t_{1}}\right)^{m} \frac{\frac{\gamma}{t_{1}}}{1-\frac{\gamma}{t_{1}}}=\frac{\gamma}{t_{1}-\gamma}\left(\frac{\gamma}{t_{1}}\right)^{m}
\end{gathered}
$$

y

$$
\sum_{j \geq l} \frac{1}{m+j}\left(\frac{\gamma}{t_{1}}\right)^{m+j} \leq \sum_{j \geq l}\left(\frac{\gamma}{t_{1}}\right)^{m+j},
$$

Por tanto se obtiene que

$$
\begin{gathered}
\sum_{n \geq m+1}\left(\frac{1}{n t_{1}^{n-1}} \sum_{k=0}^{n-1} \varphi_{k} t_{1}^{k}\right) \gamma^{n} \\
\leq \frac{t_{1}\left(\frac{\gamma}{t_{1}}\right)^{m}}{1-\frac{\gamma}{t_{1}}}\left[\sum_{n=0}^{m} t_{1}^{n} \varphi_{n}+\sum_{n \geq m+1} \varphi_{n} t_{1}^{n}\left(\frac{\gamma}{t_{1}}\right)^{n-m+1}\right] \\
\leq \frac{t_{1}\left(\frac{\gamma}{t_{1}}\right)^{m}}{1-\frac{\gamma}{t_{1}}} \sum_{n \geq 0} \varphi_{n} t_{1}^{n} .
\end{gathered}
$$

Aplicando las desigualdades de Cauchy en el intervalo $\left[0, t^{*}\right]$, siendo su extremo superior $t^{*}=t_{1}+\frac{\delta-t_{1}}{2}$, entonces podemos escribir las siguientes acotaciones

$$
\begin{aligned}
& \left\|X_{n}\right\|=\varphi_{n} \leq \frac{M_{2}}{\left(t^{*}\right)^{n}}, \quad n \geq 0, \\
& \varphi_{n} t_{1}^{n} \leq M_{2}\left(\frac{t_{1}}{t^{*}}\right)^{n}, \quad n \geq 0,
\end{aligned}
$$

y

$$
\sum_{n \geq 0} \varphi_{n} t_{1}^{n} \leq M_{2} \frac{\frac{t_{1}}{t^{*}}}{1-\frac{t_{1}}{t^{*}}}
$$

FUNC. ESPECIALES Y EC. DIF. MATRICIALES 
Aplicando las desigualdades dadas en (5.44), (5.45), y la igualdad deducida en (5.47), se sigue que el error de truncamiento de la solución matricial $X(t)$ cumple la siguiente desigualdad

$$
\begin{gathered}
\|X(t)-X(t, m)\| \\
\leq \frac{M_{1} M_{2} t_{1}}{1-\frac{\gamma}{t_{1}}}\left(\frac{\gamma}{t_{1}}\right)^{m} \frac{\frac{t_{1}}{t^{*}}}{1-\frac{t_{1}}{t^{*}}}=\frac{M_{1} M_{2} t_{1}^{3}}{\left(t_{1}-\gamma\right)\left(t^{*}-t_{1}\right)}\left(\frac{\gamma}{t_{1}}\right)^{m} .
\end{gathered}
$$

Nosotros estamos interesados en el error de truncación de la derivada $X^{\prime}(t)$, observar para ello que

$$
X^{\prime}(t)-X^{\prime}(t, m)=\sum_{n \geq m+1} n X_{n} t^{n-1} .
$$

Por la igualdad (5.49) y por la acotación dada en (5.44) relativa a $X(t)$, se tiene ahora para $X^{\prime}(t)$ que

$$
\left\|X^{\prime}(t)-X^{\prime}(t, m)\right\| \leq \sum_{n \geq m+1} \frac{M_{1}}{t_{1}^{n-1}}\left(\sum_{k=0}^{n-1} \varphi_{k} t_{1}^{k}\right) \gamma^{n-1}, \quad 0 \leq t \leq \gamma .
$$

De nuevo, por (5.5) relativo al orden de sumación de una serie doble de números complejos y por la convergencia de la serie

$$
\sum_{n \geq 1} \varphi_{n} \gamma^{n}
$$

obtenemos la siguiente representación equivalente de la serie que aparece en el miembro derecho de (5.50)

$$
\begin{gathered}
\sum_{n \geq m+1} \frac{1}{t_{1}^{n-1}}\left(\sum_{k=0}^{n-1} \varphi_{k} t_{1}^{k}\right) \gamma^{n-1} \\
=\frac{1}{\gamma}\left\{\sum_{n \geq m+1} \frac{1}{t_{1}^{n-1}}\left(\sum_{k=0}^{n-1} \varphi_{k} t_{1}^{k}\right) \gamma^{n}\right\}=
\end{gathered}
$$

FUNC. ESPECIALES Y EC. DIF. MATRICIALES 


$$
\begin{gathered}
=\frac{1}{\gamma}\left\{t_{1} \varphi_{0} \sum_{j \geq 1}\left(\frac{\gamma}{t_{1}}\right)^{m+j}+t_{1}^{2} \varphi_{1} \sum_{j \geq 1}\left(\frac{\gamma}{t_{1}}\right)^{m+j}+\ldots\right. \\
t_{1}^{m+1} \varphi_{m} \sum_{j \geq 1}\left(\frac{\gamma}{t_{1}}\right)^{m+j}+t_{1}^{m+2} \varphi_{m+1} \sum_{j \geq 2}\left(\frac{\gamma}{t_{1}}\right)^{m+j}+ \\
+t_{1}^{m+3} \varphi_{m+2} \sum_{j \geq 3}\left(\frac{\gamma}{t_{1}}\right)^{m+j}+\ldots+ \\
\left.+t_{1}^{m+l} \varphi_{m+l-1} \sum_{j \geq l}\left(\frac{\gamma}{t_{1}}\right)^{m+j}+\ldots\right\} .
\end{gathered}
$$

Luego

$$
\begin{gathered}
\sum_{n \geq m+1} \frac{1}{t_{1}^{n-1}}\left(\sum_{k=0}^{n-1} \varphi_{k} t_{1}^{k}\right) \gamma^{n-1} \\
=\frac{1}{\gamma}\left\{t_{1} \sum_{j \geq 1}\left(\frac{\gamma}{t_{1}}\right)^{m+j}\right\}\left(\varphi_{0}+t_{1} \varphi_{1}+\ldots+t_{1}^{m} \varphi_{m}\right) \\
+\frac{t_{1}}{\gamma}\left\{t_{1}^{m+1} \varphi_{m+1} \sum_{j \geq 2}\left(\frac{\gamma}{t_{1}}\right)^{m+j}+t_{1}^{m+2} \varphi_{m+2} \sum_{j \geq 3}\left(\frac{\gamma}{t_{1}}\right)^{m+j}\right. \\
\left.+\ldots+t_{1}^{m+l} \varphi_{m+l} \sum_{j \geq l+1}\left(\frac{\gamma}{t_{1}}\right)^{m+j}+\ldots\right\} .
\end{gathered}
$$

Teniendo en cuenta (5.50) y (5.48), concluimos que

$$
\left\|X^{\prime}(t)-X^{\prime}(t, m)\right\| \leq \frac{M_{1} M_{2} t_{1}^{3}}{\gamma\left(t_{1}-\gamma\right)\left(t^{*}-t_{1}\right)}\left(\frac{\gamma}{t_{1}}\right)^{m}, \quad 0 \leq t \leq \gamma .
$$

Observar que bajo las hipótesis (5.33), por [18, p.114] se tiene

$$
\begin{gathered}
\left\|V_{n}(t)\right\| \leq\|X(t, n)\| \leq\|X(t, n)-X(t)\|+\|X(t)\| \\
\leq \xi q_{0} \omega_{0}+\omega_{0} \exp \left(\gamma k_{0}\right)
\end{gathered}
$$




$$
\left\|V_{n}(t)\right\| \leq \omega_{0}\left(\xi q_{0}+\exp \left(\gamma k_{0}\right)\right), \quad 0 \leq t \leq \gamma, \quad n \geq n_{0} .
$$

Si $W(t)=V(t)[U(t)]^{-1}$ es la solución de (5.1) y

$$
W_{n}(t)=V_{n}(t)\left[U_{n}(t)\right]^{-1}=\left\{\left[\begin{array}{ll}
0 & I_{p}
\end{array}\right] X(t, n)\right\}\left\{\left[\begin{array}{ll}
I_{q} & 0
\end{array}\right] X(t, n)\right\}^{-1},
$$

es la aproximación de grado $n$ por truncación, donde

$$
X(t, m)=\sum_{k=0}^{m} X_{k} t^{k}, \quad 0 \leq t \leq \gamma,
$$

entonces

$$
\left.\begin{array}{c}
W(t)-W_{n}(t)=V(t)[U(t)]^{-1}-V_{n}(t)\left[U_{n}(t)\right]^{-1} \\
=\left[V(t)-V_{n}(t)\right][U(t)]^{-1}+V_{n}(t)\left[(U(t))^{-1}-\left(U_{n}(t)\right)^{-1}\right] .
\end{array}\right]
$$

Tomando normas en (5.54) se tiene

$$
\begin{gathered}
\left\|W(t)-W_{n}(t)\right\| \\
\left.\leq\left\|V(t)-V_{n}(t)\right\|\left\|(U(t))^{-1}\right\|+\left\|V_{n}(t)\right\|\left\|(U(t))^{-1}-\left(U_{n}(t)\right)^{-1}\right\|\right]
\end{gathered}
$$

y por el lema de Banach [21, p.28],

$$
\left\|(U(t))^{-1}-\left(U_{n}(t)\right)^{-1}\right\| \leq\left\|(U(t))^{-1}\right\|\left\|\left(U_{n}(t)\right)^{-1}\right\|\left\|U(t)-U_{n}(t)\right\| .
$$

Por (5.55), (5.56) y teniendo en cuenta que $\left\|V(t)-V_{n}(t)\right\|$ y $\left\|U(t)-U_{n}(t)\right\|$ están ambas acotadas superiormente por $\|X(t)-X(t, n)\|$, se obtiene

$$
\begin{gathered}
\left\|W(t)-W_{n}(t)\right\| \\
\leq\left\|(U(t))^{-1}\right\|\left[\left\|V(t)-V_{n}(t)\right\|+\left\|V_{n}(t)\right\|\left\|\left(U_{n}(t)\right)^{-1}\right\|\left\|U(t)-U_{n}(t)\right\|\right] \\
\leq\left\|(U(t))^{-1}\right\|\left(1+\left\|V_{n}(t)\right\|\left\|\left(U_{n}(t)\right)^{-1}\right\|\right)\|X(t)-X(t, n)\|
\end{gathered}
$$

Notar que por el anterior lema 5.2.3 y por (5.31), (5.52), para $n \geq n_{0}$, $0 \leq t \leq \gamma$, se tiene

$$
\begin{gathered}
\left\|(U(t))^{-1}\right\|\left(1+\left\|V_{n}(t)\right\|\left\|\left(U_{n}(t)\right)^{-1}\right\|\right) \\
\leq\left(\omega_{0} \xi q_{0}\right)^{-1}\left\{1+\xi^{-1} q_{0}^{-1}\left(\xi q_{0}+\exp \left(\gamma k_{0}\right)\right)\left(1-\frac{\gamma}{\delta}\right)^{-1}\right\}
\end{gathered}
$$

FUNC. ESPECIALES Y EC. DIF. MATRICIALES 
Por (5.48) y el lema 5.2.3 anterior, si tomamos $n_{1}$ el primer entero positivo tal que

$$
\left(\frac{\gamma}{t_{1}}\right)^{n}<\min \left\{\frac{\xi q_{0} \omega_{0}\left(t_{1}-\gamma\right)\left(t^{*}-t_{1}\right)}{M_{1} M_{2} t_{1}^{3}}, \frac{\varepsilon\left(t_{1}-\gamma\right)\left(t^{*}-t_{1}\right)}{M_{1} M_{2} t_{1}^{3}}\right\},
$$

entonces por (5.48) se tiene que

$$
\|X(t)-X(t, n)\| \leq \xi q_{0} \omega_{0}, \quad n \geq n_{1} ; \quad 0 \leq t \leq \gamma
$$

$\mathrm{y}$

$$
\|X(t)-X(t, n)\|<\varepsilon, \quad n \geq n_{1} ; \quad 0 \leq t \leq \gamma .
$$

Por (5.51) y por el lema 5.2.3 de la sección anterior, si tomamos $n_{2}$ como el primer entero positivo $n$ tal que

$$
\left(\frac{\gamma}{t_{1}}\right)^{n}<\min \left\{\frac{\delta^{-1} \gamma \xi q_{0} \omega_{0}\left(t_{1}-\gamma\right)\left(t^{*}-t_{1}\right)}{M_{1} M_{2} t_{1}^{3}}, \frac{\varepsilon \gamma\left(t_{1}-\gamma\right)\left(t^{*}-t_{1}\right)}{M_{1} M_{2} t_{1}^{3}}\right\}
$$

entonces por $(5.51),(5.62)$, se concluye que

$$
\left\|X^{\prime}(t)-X^{\prime}(t, n)\right\|<\delta^{-1} \xi q_{0} \omega_{0}, \quad n \geq n_{2}, \quad 0 \leq t \leq \gamma .
$$

$\mathrm{y}$

$$
\left\|X^{\prime}(t)-X^{\prime}(t, n)\right\|<\varepsilon, \quad n \geq n_{2}, \quad 0 \leq t \leq \gamma .
$$

Sin embargo, notemos que como $\gamma \delta^{-1}<1$, el primer término del miembro derecho de la desigualdad (5.62) es menor que

$$
\frac{\xi q_{0} \omega_{0}\left(t_{1}-\gamma\right)\left(t^{*}-t_{1}\right)}{M_{1} M_{2} t_{1}^{3}}
$$

lo cual aparece en (5.59). Por tanto, si $n$ satisface (5.62) y

$$
\left(\frac{\gamma}{t_{1}}\right)^{n}<\frac{\varepsilon\left(t_{1}-\gamma\right)\left(t^{*}-t_{1}\right)}{M_{1} M_{2} t_{1}^{3}}
$$

se cumple también (5.59). Ambas condiciones, (5.62) y (5.65) pueden escribirse en la forma

$$
n>\frac{\ln \left[\frac{\left(t_{1}-\gamma\right)\left(t^{*}-t_{1}\right)}{M_{1} M_{2} t_{1}^{3}} \operatorname{mín}\left\{\varepsilon, \gamma \varepsilon, \delta^{-1} \gamma \xi q_{0} \omega_{0}\right\}\right]}{\ln \left(\frac{\gamma}{t_{1}}\right)} .
$$

Resumiendo los resultados de este apartado y el lema 5.2.2 del apartado segundo podemos establecer el siguiente resultado:

FUNC. ESPECIALES Y EC. DIF. MATRICIALES 
Teorema 5.3.1 . Consideremos el problema de valor inicial (5.1) donde los coeficientes matriciales son funciones continuas en el intervalo $0 \leq t<c$. Sean $k_{0}, q_{0}, \omega_{0}$ y $\delta$ los definidos en el lema 5.2.1, sea $0<\gamma<\delta$ y $\xi>0$ con

$$
\gamma \exp \left(\gamma k_{0}\right)=\left[q_{0} \omega_{0}\right]^{-1}-\xi .
$$

Sea $X(t)$ la solución analítica del problema (5.3) y sea $V(t)=\left[\begin{array}{ll}0 & I_{p}\end{array}\right] X(t)$, $U(t)=\left[\begin{array}{ll}I_{q} & 0\end{array} X(t)\right.$ y sea $W(t)=V(t)[U(t)]^{-1}$ la solución de (5.1) en $[0, \delta]$. Sea $t_{1}$ de manera que $\gamma<t_{1}<\delta, t^{*}=t_{1}+\frac{1}{2}\left(\delta-t_{1}\right)$ y sea $\varepsilon>0$ un error admisible. Sea $n_{1}$ el primer entero $n$ verificando (5.66), sean $M_{1}$ y $M_{2}$ los definidos por las expresiones (5.41) y (5.21) respectivamente, sean $V_{n}(t)$ y $U_{n}(t)$ definidos por

$$
V_{n}(t)=\left[\begin{array}{ll}
0 & I_{p}
\end{array}\right] X(t, n), \quad U_{n}(t)=\left[\begin{array}{ll}
I_{q} & 0
\end{array}\right] X(t, n),
$$

donde $X(t, n)$ es la truncación de orden $n$ de $X(t)$ definida por (5.39). Si

$$
\left.\begin{array}{c}
W_{n}(t)=V_{n}(t)\left[U_{n}(t)\right]^{-1} \\
=\left\{\left[\begin{array}{ll}
0 & I_{p}
\end{array}\right] \sum_{k=0}^{n} X_{k} t^{k}\right\}\left\{\left[\begin{array}{ll}
I_{q} & 0
\end{array}\right] \sum_{k=0}^{n} X_{k} t^{k}\right\}^{-1}, \quad 0 \leq t \leq \gamma, \quad n \geq n_{1}
\end{array}\right]
$$

donde

$$
X_{0}=\left[\begin{array}{c}
I_{q} \\
W_{0}
\end{array}\right], \quad X_{n+1}=\frac{1}{(n+1)} \sum_{k=0}^{n} S_{n-k} X_{k}, \quad n \geq 0,
$$

$y$

$$
S(t)=\sum_{k \geq 0} S_{k} t^{k}, \quad 0 \leq t<\delta,
$$

entonces se cumple

$$
\left\|W(t)-W_{n}(t)\right\|<\varepsilon, \quad n \geq n_{1}, \quad 0 \leq t \leq \gamma .
$$

Veamos un ejemplo, sobre el que testearemos nuestras conclusiones.

Ejemplo 1. Consideremos el problema de valor inicial

$$
W^{\prime}=W+\sin (t) W^{2}, \quad W(0)=1 .
$$

FUNC. ESPECIALES Y EC. DIF. MATRICIALES 
El problema lineal asociado del tipo (5.3) toma en este caso particular la forma

$$
\begin{gathered}
X^{\prime}(t)=\left[\begin{array}{cc}
0 & -\sin (t) \\
0 & 1
\end{array}\right] X(t), X(0)=\left[\begin{array}{l}
1 \\
1
\end{array}\right] \\
S(t)=\sum_{n \geq 0} S_{n} t^{n} ; S_{0}=\left[\begin{array}{cc}
0 & 0 \\
0 & 1
\end{array}\right] ; \\
S_{2 k}=0, k \geq 1 ; S_{2 k+1}=\frac{(-1)^{k+1}}{(2 k+1) !}\left[\begin{array}{cc}
0 & 1 \\
0 & 0
\end{array}\right], k \geq 0 .
\end{gathered}
$$

La solucion exacta de (5.70) es

$$
W(t)=\frac{2 e^{t}}{1-e^{t}(\sin (t)-\cos (t))}
$$

Sea $c=1,03841563726656$. En este caso las constantes del teorema 5.3.1 son

$$
\begin{array}{lll}
\omega_{0}=\sqrt{2} & k_{0}=1,319845 \\
q_{0}=0,861598 & \gamma=0,1 \\
\xi=0,706583 & t_{1}=0,2 \\
\delta & =0,4 & t^{*}=0,3
\end{array}
$$

y el grado de truncación $n_{1}$ de la solución aproximada $W_{n_{1}}(t)$ con error menor que $\epsilon$ para diferentes valores de $\epsilon$, según (5.66), se da en la tabla siguiente. Para ello es necesario calcular previamente las cotas

$$
M_{1}=1,1, \quad M_{2}=13,9
$$

Para los valores $\epsilon=10^{-1}, \epsilon=10^{-2}, \epsilon=10^{-3}, \epsilon=10^{-4}$, el valor de $n_{1}$ no

\begin{tabular}{|c|c|c|c|c|c|c|}
\hline \hline$\epsilon$ & $10^{-1}$ & $10^{-2}$ & $10^{-3}$ & $10^{-4}$ & $10^{-5}$ & $10^{-6}$ \\
\hline$n_{1}$ & 14 & 14 & 14 & 14 & 15 & 18 \\
\hline \hline
\end{tabular}

Cuadro 5.1: Orden de truncación. Ejemplo 1 
cambia porque en (5.66) se tiene

$$
\min \left\{\delta^{-1} \gamma \xi q_{0} \omega_{0}, \varepsilon, \gamma \varepsilon\right\}=\delta^{-1} \gamma \xi q_{0} \omega_{0}
$$

Nota 1. El método presentado aquí para el caso en que los coeficientes son analíticos puede ser visto como una versión continua del método dado por Schiff and Shnider en [57]. Los métodos de un paso propuestos en [57] para la solución numérica de (5.1) toman la forma (ver expresión (27) de [57])

$$
W\left(t_{0}+h\right)=\left[\tilde{\alpha}\left(t_{0}, h\right) W_{0}+\tilde{\beta}\left(t_{0}, h\right)\right]\left[\tilde{\gamma}\left(t_{0}, h\right) W_{0}+\tilde{\delta}\left(t_{0}, h\right)\right]^{-1}
$$

donde

$$
\tilde{\Gamma}\left(t_{0}, h\right)=\left[\begin{array}{ll}
\tilde{\alpha}\left(t_{0}, h\right) & \tilde{\beta}\left(t_{0}, h\right) \\
\tilde{\gamma}\left(t_{0}, h\right) & \tilde{\delta}\left(t_{0}, h\right)
\end{array}\right]
$$

es una aproximación a la solución fundamental $\Gamma\left(t_{0}, h\right)$ del problema (5.3). Observar que si $S(t)$ está dada por $(5.3)$ y $S(t, n)$ es la suma parcial n-ésima del desarrollo de Taylor de $S(t)$, entonces tomando $t_{0}=0$, si $\Gamma_{n}(t)$ es la solución fundamental del sistema truncado

$$
\Gamma_{n}^{\prime}(t)=S(t, n) \Gamma_{n}(t), \quad \Gamma_{n}(0)=I
$$

y $\Gamma(t)$ es la solución fundamental satisfaciendo

$$
\Gamma^{\prime}(t)=S(t) \Gamma(t), \quad \Gamma(0)=I,
$$

entonces por $\left[18\right.$, p.78] se deduce que $\left\{\Gamma_{n}\right\}$ converge uniformemente a $\Gamma$ en un entorno de $t_{0}=0$. Esto significa que $\Gamma_{n}(t)$ puede ser vista como una aproximación de $\Gamma(t)$. Luego la aproximación $W_{n}(t)$ del problema (5.1) dada por el teorema 5.3.1 y definida por $W_{n}(t)=V_{n}(t)\left[U_{n}(t)\right]^{-1}$ puede ser escrita en la forma

$$
\begin{aligned}
W_{n}(t)= & \left\{\left[\begin{array}{ll}
0 & I
\end{array}\right] X(t, n)\right\}\left\{\left[\begin{array}{ll}
I & 0
\end{array}\right] X(t, n)\right\}^{-1}=V_{n}(t)\left[U_{n}(t)\right]^{-1} \\
& =\left\{\alpha_{n}(t) W_{0}+\beta_{n}(t)\right\}\left\{\gamma_{n}(t) W_{0}+\delta_{n}(t)\right\}^{-1}
\end{aligned}
$$

donde

$$
\Gamma_{n}(t)=\left[\begin{array}{ll}
\alpha_{n}(t) & \beta_{n}(t) \\
\gamma_{n}(t) & \delta_{n}(t)
\end{array}\right]
$$

Así, aunque no construimos $W_{n}(t)$ por aproximación de $\Gamma(t)$, la expresión de $W_{n}(t)$ es equivalente a la dada en [57] y así conserva las ventajas de tal método en el dominio considerado sin singularidades.

FUNC. ESPECIALES Y EC. DIF. MATRICIALES 


\subsection{Sobre la estabilidad del método}

En este apartado analizamos algunas características adicionales relacionadas con el método propuesto en el apartado anterior. Dado que la solución aproximada construida se basa en la determinación previa del intervalo, donde con la notación anterior, $U(t)=\left[\begin{array}{ll}I_{q} & 0\end{array}\right] X(t)$ es invertible, algunas preguntas surgen de un modo natural. "Es la solución prolongable a un intervalo mayor desde la parte derecha del dominio de existencia?. "Cuál es el comportamiento de la longitud del nuevo dominio extendido después de más aplicaciones del método?. "Cuál es el error adicional cuando en las sucesivas aplicaciones aproximamos el valor teórico exacto del lado izquierdo del nuevo intervalo de trabajo?. Estos puntos que describen la estabilidad del método son tratados a continuación.

Sea $X(t)$ la solución del problema (5.3) y sea $U(t)=\left[\begin{array}{ll}I_{q} & 0\end{array}\right] X(t)$. Supongamos $0<t_{0}<c$ con $U\left(t_{0}\right)$ invertible. Si $\left\|U(t)-U\left(t_{0}\right)\right\|<\left\|\left(U\left(t_{0}\right)\right)^{-1}\right\|^{-1}$, entonces por el lema de perturbación [15, p.538] se sigue que

$$
\left\|(U(t))^{-1}\right\|<\left\|\left(U\left(t_{0}\right)\right)^{-1}\right\|\left(1-\left\|U(t)-U\left(t_{0}\right)\right\|\left\|\left(U\left(t_{0}\right)\right)^{-1}\right\|\right)^{-1}
$$

Por el teorema del valor medio [14, p.158], se deduce que

$$
\left\|U(t)-U\left(t_{0}\right)\right\| \leq\left|t-t_{0}\right| \sup \left\{\left\|U^{\prime}(s)\right\| ; t_{0} \leq s \leq t\right\} .
$$

Desde aquí, si

$$
M\left(\delta, t_{0}\right)=\sup \left\{\left\|U^{\prime}(s)\right\| \quad ; \quad t_{0} \leq s<t_{0}+\delta\right\} \quad \delta>0,
$$

y

$$
\delta M\left(\delta, t_{0}\right)<\left\|\left(U\left(t_{0}\right)\right)^{-1}\right\|^{-1},
$$

entonces

$$
U(t) \text { es invertible en } t_{0} \leq t<t_{0}+\delta \text {, }
$$

y por (5.73)-(5.74) se deduce de (5.71)

$$
\left\|(U(t))^{-1}\right\|<\left\|\left(U\left(t_{0}\right)\right)^{-1}\right\|\left(1-\left\|\left(U\left(t_{0}\right)\right)^{-1}\right\| \delta M\left(\delta, t_{0}\right)\right)^{-1} .
$$

FUNC. ESPECIALES Y EC. DIF. MATRICIALES 
Notar que por (5.73) y (5.29) se sigue que

$$
\left.\begin{array}{c}
M\left(\delta, t_{0}\right)=\sup \left\{\|[A(s) B(s)] X(s)\| ; t_{0} \leq s \leq t_{0}+\delta\right\} \\
\leq q\left(t_{0}\right) e^{\delta K\left(t_{0}\right)}\left(1+\left\|W\left(t_{0}\right)\right\|^{2}\right)^{\frac{1}{2}}
\end{array}\right]
$$

donde

$$
\begin{gathered}
q\left(t_{0}\right)=\operatorname{máx}\left\{\|[A(s) \quad B(s)]\| ; \quad t_{0} \leq s \leq c\right\} \\
K\left(t_{0}\right)=\operatorname{máx}\left\{\|S(t)\| ; t_{0} \leq t \leq c\right\} .
\end{gathered}
$$

Por tanto la condición (5.74) se verifica si

$$
\delta q\left(t_{0}\right) e^{\delta K\left(t_{0}\right)}\left(1+\left\|W\left(t_{0}\right)\right\|^{2}\right)^{\frac{1}{2}}<1 .
$$

Esta desigualdad motiva la siguiente definición:

Definición 5.4.1 . Sea $U(t)=\left[\begin{array}{ll}I_{q} & 0\end{array}\right] X(t)$ donde $X(t)$ es la solución de (5.3) definida en $[0, c]$, y sea $t^{*}$ un punto $0<t^{*}<c$, donde $U\left(t^{*}\right)$ es invertible. Llamamos longitud de invertibilidad crítica de $U$ en $t^{*}$, al número $\delta\left(t^{*}\right)>0$ con $t^{*}+\delta\left(t^{*}\right)<c$ tal que

$$
\delta\left(t^{*}\right) e^{\delta\left(t^{*}\right) K\left(t^{*}\right)} q\left(t^{*}\right)\left(1+\left\|W\left(t^{*}\right)\right\|^{2}\right)^{\frac{1}{2}}=1 .
$$

Si $t^{*}+\delta\left(t^{*}\right)>c$ entonces la solución está ya definida en todo el dominio. De aquí, si $t^{*}+\delta\left(t^{*}\right) \leq c$, entonces la solución $W$ del problema (5.1) es prolongable en el intervalo $\left[t^{*}, t^{*}+\delta\left(t^{*}\right)[\right.$.

Ahora consideremos la sucesión de puntos

$$
0=t_{0}<t_{1}<t_{2}<\ldots<t_{j}<c,
$$

donde $U\left(t_{i}\right)$ es invertible y $t_{i}+\delta\left(t_{i}\right)<c$ para $0 \leq i \leq j$. Introduzcamos las constantes

$$
\begin{gathered}
q\left(t_{i}\right)=\operatorname{máx}\left\{\|\left[\begin{array}{ll}
A(s) & \left.B(s)] \| \quad ; \quad t_{i} \leq s \leq c\right\} \\
K\left(t_{i}\right)=\operatorname{máx}\left\{\|S(t)\| \quad ; \quad t_{i} \leq t \leq c\right\} .
\end{array}\right]\right.
\end{gathered}
$$


Por (5.77) se tiene

$$
\frac{\delta\left(t_{j}\right)}{\delta\left(t_{j-1}\right)}=\frac{q\left(t_{j-1}\right)}{q\left(t_{j}\right)} \exp \left(\delta\left(t_{j-1}\right) K\left(t_{j-1}\right)-\delta\left(t_{j}\right) K\left(t_{j}\right)\right)\left[\frac{1+\left\|W\left(t_{j-1}\right)\right\|^{2}}{1+\left\|W\left(t_{j}\right)\right\|^{2}}\right]^{\frac{1}{2}}
$$

Luego

$$
\begin{gathered}
\frac{\delta\left(t_{j}\right)}{\delta(0)}=\frac{q(0)}{q\left(t_{j}\right)} \exp \left(\delta(0) K(0)-\delta\left(t_{j}\right) K\left(t_{j}\right)\right)\left[\frac{1+\|W(0)\|^{2}}{1+\left\|W\left(t_{j}\right)\right\|^{2}}\right]^{\frac{1}{2}} \\
\geq \frac{q(0)}{q\left(t_{j}\right)} \exp \left(K(0)\left(\delta(0)-\delta\left(t_{j}\right)\right)\right)\left[\frac{1+\|W(0)\|^{2}}{1+\left\|W\left(t_{j}\right)\right\|^{2}}\right]^{\frac{1}{2}} \cdot \\
\delta\left(t_{j}\right) \geq \frac{\delta(0) q(0) \exp (K(0)(\delta(0)-c))}{q\left(t_{j}\right)}\left[\frac{1+\|W(0)\|^{2}}{1+\left\|W\left(t_{j}\right)\right\|^{2}}\right]^{\frac{1}{2}}, j \geq 1 \\
t_{j}+\delta\left(t_{j}\right) \leq c, \quad j \geq 1 .
\end{gathered}
$$

La desigualdad (5.80) proporciona una medida de la prolongabilidad de la solución $W$ del problema (5.1) en $t=t_{j}$ en términos de los datos y del valor de la solución en $t=t_{j}$.

Desde (5.80) podemos obtener algunas conclusiones muy útiles con objeto de extender el dominio de existencia de la solución $W$ del problema (5.1). Primero, el intervalo inicial de trabajo $[0, c]$ no debería ser grande porque el número $\exp (K(0)[\delta(0)-c])$ decrece con $c$. Además, si la norma $\left\|W\left(t_{j}\right)\right\|$ decrece con respecto a $\|W(0)\|$ la razón $\frac{\delta\left(t_{j}\right)}{\delta(0)}$ crece. En particular, si $W_{0}=0$, el procedimiento de prolongabilidad está peor condicionado que en otro caso. Además, para el caso en que el tiempo sea invariante, la inecuación (5.80) toma la forma

$$
\delta\left(t_{j}\right) \geq \delta(0) e^{(\delta(0)-c) K(0)}\left[\frac{1+\left\|W_{0}\right\|^{2}}{1+\left\|W\left(t_{j}\right)\right\|^{2}}\right]^{\frac{1}{2}} j \geq 1 .
$$

FUNC. ESPECIALES Y EC. DIF. MATRICIALES 
Esta desigualdad es cierta para el caso en que los coeficientes sean variables porque $\frac{q(0)}{q\left(t_{j}\right)} \geq 1$, y es particularmente interesante cuando se conoce una cota de error a priori de la solución $W$. En efecto, si $\|W(t)\| \leq M$ para todo $t$ en $[0, c]$, entonces por $(5.81)$ se tiene

$$
\delta\left(t_{j}\right) \geq \delta(0) e^{(\delta(0)-c) K(0)}\left[\frac{1+\left\|W_{0}\right\|^{2}}{1+M^{2}}\right]^{\frac{1}{2}} .
$$

Esta desigualdad proporciona una cota inferior para el error a priori de la longitud de invertibilidad crítica y así si $\Omega$ es el miembro derecho de (5.82), tomando el primer número positivo $m$ de modo que $m \Omega>c$, se cubre todo el dominio $[0, c]$.

El siguiente ejemplo garantiza que la solución de un modelo particular de ecuaciones del tipo (5.1) puede obtenerse siempre después de la haber aplicado el método un número finito de veces.

Ejemplo 1. Consideremos el problema (5.1) para el caso $B(t)=0$ :

$$
W^{\prime}(t)=C(t)-D(t) W(t)-W(t) A(t) \quad, \quad W(0)=W_{0} .
$$

Como el problema (5.83) es equivalente a la ecuación integral

$$
W(t)=W_{0}+\int_{0}^{t}\{C(s)-D(s) W(s)-W(s) A(s)\} d s \quad, \quad 0 \leq t \leq c .
$$

tomando normas en la ecuación y denotando por $g(t)=\|W(t)\|$, se deduce que

$$
g(t) \leq\left\|W_{0}\right\|+\int_{0}^{c}\|C(s)\| d s+\int_{0}^{t} g(s)(\|D(s)\|+\|A(s)\|) d s .
$$

Por (5.84) y el lema de Gronwall [18, p.95] se sigue

$$
\left.\begin{array}{c}
\|W(t)\| \leq\left(\left\|W_{0}\right\|+\int_{0}^{c}\|C(s)\| d s\right) . \\
\cdot \exp \left(\int_{0}^{c}(\|A(s)\|+\|D(s)\|) d s\right)=M, \quad 0 \leq t \leq c,
\end{array}\right]
$$

FUNC. ESPECIALES Y EC. DIF. MATRICIALES 
Si $\Omega$ es el miembro derecho de (5.82) y $M$ está dado por (5.85), entonces tomando el primer entero positivo $m$ de modo que $m \Omega \geq c$, la solución proporcionada por el método es prolongable en todo el dominio $[0, c]$ después de a lo sumo $m$ iteraciones.

Ejemplo 2. Consideremos el problema unidimensional

$$
W^{\prime}=W+\sin (t) W^{2}, \quad W(0)=1,
$$

cuya solución exacta está definida en $0 \leq t \leq c=1,03841563726656$ por

$$
W(t)=\frac{2 e^{t}}{1-e^{t}(\sin (t)-\cos (t))} .
$$

En este caso tenemos

$$
S=\left[\begin{array}{cc}
0 & -\sin (t) \\
0 & 1
\end{array}\right] \quad, \quad K_{0}=\operatorname{máx}\{\|S(t)\| ; \quad 0 \leq t \leq c\}=1,31984576369327 \text {. }
$$

Considerando la ecuación (5.77) se tiene

$$
\begin{gathered}
\delta\left(t_{0}\right)=\delta(0)>0,4010308655321=t_{1}, \\
\delta\left(t_{1}\right)>0,20359355233278=t_{2} \\
\delta\left(t_{2}\right)>0,24654713969143=t_{3} \\
\delta\left(t_{3}\right)>0,23816773231748=t_{4}
\end{gathered}
$$

con

$$
\sum_{i=0}^{3} \delta\left(t_{i}\right)>c
$$

esto significa que después de cuatro aplicaciones del método se cubre el dominio donde $W$ no tiene singularidades.

Ejemplo 3. Consideremos el problema de Ricatti

$$
W^{\prime}=W^{2} \quad, \quad W(0)=1,
$$


cuya solución exacta es $W(t)=-(t-1)^{-1}$ en [0,1[. En este caso tenemos $K_{0}=q_{0}=1, w_{0}=\sqrt{2}$ y considerando la ecuación (5.77) se tiene

$$
\begin{gathered}
\delta(0)=\delta\left(t_{0}\right)>0,45060051586483=t_{1}, \\
\delta\left(t_{1}\right)>0,177139328564488=t_{2}, \\
\delta\left(t_{2}\right)>0,23375041210495=t_{3}, \\
\delta\left(t_{3}\right)>0,22375274305064=t_{4},
\end{gathered}
$$

con

$$
\sum_{i=0}^{3} \delta\left(t_{i}\right)>1 .
$$

Luego, tras cuatro aplicaciones del método, el intervalo [0,1[ está cubierto.

Concluimos este apartado estudiando el error adicional cuando se prolonga la solución $W$ de (5.1) al intervalo $\left[t_{1}, t_{1}+\delta\left(t_{1}\right)[\right.$, donde se tiene que $0<t_{1}<t_{1}+\delta\left(t_{1}\right) \leq c$ y $U\left(t_{1}\right)=\left[\begin{array}{ll}I_{q} & 0\end{array}\right] X\left(t_{1}\right)$ es invertible. Con objeto de aplicar de nuevo el método empezando desde el punto $t_{1}$, debemos tener en cuenta que el valor $W\left(t_{1}\right)$ de la solución exacta de (5.1) en $t=t_{1}$, no está disponible. En su lugar, tenemos un valor aproximado $W_{n_{1}}\left(t_{1}\right)=\tilde{W}_{1}$ procedente de la aplicación del método en el intervalo $\left[0, t_{1}\right]$.

Sea $n_{1}$ suficientemente grande para que en concordancia con (5.61)

$$
\left.\begin{array}{l}
\left\|X\left(t_{1}\right)-X\left(t_{1}, n_{1}\right)\right\|<\rho \\
0 \\
\left.\left.0 I_{p}\right] X\left(t_{1}, n_{1}\right)\right\}\left\{\left[\begin{array}{ll}
I_{q} & 0
\end{array}\right] X\left(t_{1}, n_{1}\right)\right\}^{-1}
\end{array}\right]
$$

y $X\left(t_{1}, n_{1}\right)$ es la $n_{1}-$ sima suma parcial de la solución $X(t)$ de (5.3). Consideremos el problema de valor inicial

$$
\begin{gathered}
W^{\prime}(t)=C(t)-D(t) W(t)-W(t) A(t)-W(t) B(t) W(t), \quad W\left(t_{1}\right)=\tilde{W}_{1} \\
t_{1} \leq t \leq c .
\end{gathered}
$$

FUNC. ESPECIALES Y EC. DIF. MATRICIALES 
Observar que debemos de considerar el desarrollo en serie de Taylor de $S(t)$ en $t_{1}$. El sistema lineal subyacente es ahora

$$
X^{\prime}\left(t, t_{1}\right)=S(t) X\left(t, t_{1}\right) \quad, \quad X\left(t, t_{1}\right)=\left[\begin{array}{c}
I \\
\tilde{W}_{1}
\end{array}\right] \quad, \quad t_{1} \leq t \leq c .
$$

y la solución de (5.87) está definida por

$$
W\left(t, t_{1}\right)=\left\{\left[\begin{array}{ll}
0 & I_{p}
\end{array}\right] X(t)\right\}\left\{\left[\begin{array}{ll}
I_{q} & 0
\end{array}\right] X(t)\right\}^{-1}=V\left(t, t_{1}\right)\left(U\left(t, t_{1}\right)\right)^{-1} .
$$

en el intervalo $\left[t_{1}, t_{1}+\delta\left(t_{1}\right)\right.$ [ donde $U\left(t, t_{1}\right)$ es invertible. Observar que $\tilde{W}_{1}$ es invertible por el teorema 5.3.1. En efecto, $\tilde{W}_{1}=W_{n_{1}}\left(t_{1}\right)$ siendo $n_{1}$ algún entero positivo satisfaciendo (5.66). Por el lema de Gronwall [18, p.95] la diferencia entre la solución $X(t)$ del problema (5.3) definida en $\left[t_{1}, t_{1}+\delta\left(t_{1}\right)[\right.$ y $X\left(t, t_{1}\right)$ de (5.88), verifica

$$
\begin{gathered}
g(t)=\left\|X(t)-X\left(t, t_{1}\right)\right\|, \\
g(t) \leq\left\|X\left(t_{1}\right)-X\left(t_{1}, n_{1}\right)\right\|+\int_{t_{1}}^{t}\|S(s)\| g(s) d s,
\end{gathered}
$$

y por (5.86)

$$
\left\|X(t)-X\left(t, t_{1}\right)\right\| \leq \rho \exp \left(\left(c-t_{1}\right) K\left(t_{1}\right)\right) \quad, \quad t_{1} \leq t<t_{1}+\delta\left(t_{1}\right),
$$

donde $K\left(t_{1}\right)$ está definida por (5.78). De aquí $V\left(t, t_{1}\right)=\left[\begin{array}{ll}0 & I_{p}\end{array}\right] X\left(t, t_{1}\right)$ verifica

$$
\left.\begin{array}{c}
\left\|U(t)-U\left(t, t_{1}\right)\right\| \leq\left\|X(t)-X\left(t, t_{1}\right)\right\| \leq \rho \exp \left(\left(c-t_{1}\right) K\left(t_{1}\right)\right) \\
\left\|V(t)-V\left(t, t_{1}\right)\right\| \leq\left\|X(t)-X\left(t, t_{1}\right)\right\| \leq \rho \exp \left(\left(c-t_{1}\right) K\left(t_{1}\right)\right)
\end{array}\right]
$$

Por el lema de Banach se sigue que

$$
U(t)=\left[\begin{array}{ll}
I_{q} & 0
\end{array}\right] X(t) \quad, \quad U\left(t, t_{1}\right)=\left[\begin{array}{ll}
I_{q} & 0
\end{array}\right] X\left(t, t_{1}\right)
$$

verifican

$$
\left\|(U(t))^{-1}-\left(U\left(t, t_{1}\right)\right)^{-1}\right\| \leq\left\|U(t)-U\left(t, t_{1}\right)\right\|\left\|(U(t))^{-1}\right\|\left\|\left(U\left(t, t_{1}\right)\right)^{-1}\right\|
$$


Por la demostración del lema 5.2.2, ver (5.22) se tiene

$$
\begin{gathered}
\left\|\left(U\left(t, t_{1}\right)\right)^{-1}\right\| \leq\left(1-\delta\left(t_{1}\right) q\left(t_{1}\right) \omega_{1} e^{\delta\left(t_{1}\right) K\left(t_{1}\right)}\right)^{-1} t_{1} \leq t<t_{1}+\delta\left(t_{1}\right) \\
\omega_{1}=\sqrt{1+\left\|\tilde{W}_{1}\right\|^{2}} .
\end{gathered}
$$

Por (5.90)-(5.92) se sigue que para $t_{1} \leq t<t_{1}+\delta\left(t_{1}\right)$

$$
\left.\begin{array}{c}
\left\|(U(t))^{-1}-\left(U\left(t, t_{1}\right)\right)^{-1}\right\| \leq \rho e^{K\left(t_{1}\right)\left(c-t_{1}\right)} \cdot \\
\cdot\left[\left(1-\delta(0) q(0) \omega_{0} e^{\delta(0) K(0)}\right)\left(1-\delta\left(t_{1}\right) q\left(t_{1}\right) \omega_{1} e^{\delta\left(t_{1}\right) K\left(t_{1}\right)}\right)\right]^{-1}
\end{array}\right]
$$

Por (5.89)-(5.94) la diferencia entre las solución del problema (5.1) y la solución del problema (5.87), para $t_{1} \leq t<t_{1}+\delta\left(t_{1}\right)$ verifica

$$
\begin{gathered}
\left\|W\left(t, t_{1}\right)-W(t)\right\| \\
\leq\left\|V\left(t, t_{1}\right)-V(t)\right\|\left\|\left(U\left(t, t_{1}\right)\right)^{-1}\right\| \\
+\|V(t)\|\left\|\left(U\left(t, t_{1}\right)\right)^{-1}-(U(t))^{-1}\right\| \\
\leq \rho e^{\delta(0) K(0)}\left(1-\delta\left(t_{1}\right) q\left(t_{1}\right) \omega_{1} e^{1-\delta\left(t_{1}\right) K\left(t_{1}\right)}\right)^{-1} . \\
\cdot\left[1+\omega_{0} e^{\delta(0) K(0)}\left(1-\delta(0) q(0) \omega_{0} e^{(\delta(0) K(0))}\right)^{-1}\right] .
\end{gathered}
$$

La última expresión proporciona el error adicional cuando se aplica el método en el intervalo $\left[t_{1}, t_{1}+\delta\left(t_{1}\right)[\right.$, teniendo en cuenta el error en el cálculo de la condición inicial $W\left(t_{1}\right)$, la cual es desconocida y la cual ha sido aproximada por el método en el dominio $\left[0, t_{1}\right]$. 


\section{Parte IV}

\section{BIBLIOGRAFIA}




\section{Bibliografía}

[1] H. ABOU-KANDIL, Elaboration de structures de commande hiérarchisées: Approches mono-critère et multicritère, These de Doctorat d'Etat, Univ. Pierre et Marie Curie-Paris 6, 1986.

[2] U.M. ASCHER, R.M. MATTHEIJ and R.D. RUSSELL, Numerical Solution of Boundary Value Problems for Ordinary Differential Equations, Prentice-Hall, 1988.

[3] A. BAGHI, Stackelberg differential games in economic models, Lectures Notes in Control and Information Sciences, Springer, Berlin, 1984.

[4] M. BALAS, Trends in large space structure control theory: Fondest hopes, wildest dreams, IEEE Trans. Automatic Control, Vol. 27 (1982), 522-535.

[5] A. BARRAUD, A new numerical solution of $\dot{X}=A_{1} X+X A_{2}+$ $D ; \quad X(0)=C$, IEEE Trans. Automatic Control, Vol. 22 (1977), 976977 .

[6] C. BREZINSKI, Other manifestations of the Schur complement, Linear Algebra Appl. 111 (1988), 231-247.

[7] J.L. CASTI, Dynamical Systems and Their Applications: Linear Theory, Academic Press, New York, 1977.

[8] S. CLEMHOUT and H.Y. WAN Jr, Interactive economic dynamics and differential games, J. Optim. Theory Appl. 27 (1979), 7-30.

[9] R. COMPANY, Soluciones Explícitas de Ecuaciones Diferenciales Matriciales con Coeficientes Variables, Tesis Doctoral, Departamento de Matemática Aplicada, Universidad Politécnica de Valencia, 1993. 
[10] A.G. CONSTANTINE and R.J. MUIRHEAD, Partial differential equations for hypergeometric functions of two arguments matrix, J. Multivariate Anal. 3 (1972), 332-338.

[11] J.B. CRUZ and C.I. CHEN, Series Nash solution of two-person nonzero sum linear quadratic games, J. Optim. Theory Appl. 7 (1971), 240257.

[12] E. DAVISON, The numerical solution of $\dot{X}=A_{1} X+X A_{2}+D ; \quad X(0)=$ $C$, IEEE Trans. Automatic Control, Vol. 20 (1975), 566-567.

[13] L. DIECI, Numerical integration of the differential Riccati equation and some related issues, SIAM J. Numerical Anal. 29, (1992), 781-815.

[14] J. DIEUDONNÉ, Foundations of Modern Analysis, Academic Press, New York, 1960.

[15] N. DUNFORD and J. SCHWARTZ, Linear Operators, part I, Interscience, New York, 1957.

[16] A.J. DURAN and P. LOPEZ-RODRIGUEZ, Orthogonal matrix polynomials: zeros and Blumenthal's theorem, J. of Approx. Theory 84 (1996), 96-118.

[17] M.V. FERRER, Construcción de Soluciones Explícitas de Problemas Implícitos de Orden Superior para Ecuaciones Diferenciales y en Diferencias, Tesis Doctoral, Departamento de Matemática Aplicada, Universidad Politécnica de Valencia, 1993.

[18] T.M. FLETT, Differential Analysis, Cambridge Univ. Press, Cambridge, 1980.

[19] H.I. FREEDMAN, An explicit estimate of the norm of the inverse of a matrix, SIAM Rev. 11, (1969), 254-256.

[20] Z. GAJIC and T.J. QURESHI, Lyapunov Matrix Equations in Systems Stability and Control, Academic Press, New York, 1995.

[21] G.H. GOLUB and C.F. VAN LOAN, Matrix Computations, The Johns Hopkins Univ. Press, Baltimore, 1991.

FUNC. ESPECIALES Y EC. DIF. MATRICIALES 
[22] I.S. GRADSHTEYN and I.M. RYZHIK, Table of Integrals, Series and Products, Fifth Ed., Academic Press, New York, 1994.

[23] E. HILLE, Lectures on Ordinary Differential Equations, AddisonWesley, New York, 1969.

[24] R.A. HORN and C.R. JOHNSON, Matrix Analysis, Cambridge Univ. Press, 1993.

[25] W. HOSKINS, D. MEEK and D. WALTON, The numerical solution of $\dot{X}=A_{1} X+X A_{2}+D ; \quad X(0)=C$, IEEE Trans. Automatic Control, Vol. 22 (1977), 881-882.

[26] A. ISERLES and S.P. NORSETT, On the solution of linear differential equations in Lie groups, Tech. Rep. DAMPT 1997/NA3, Univ. of Cambridge.

[27] A. ISERLES, Solving linear ordinary differential equations by the exponentials of iterated commutators. Numer. Math. 45 (1984), 183-199.

[28] A.T. JAMES, Special functions of matrix and single argument in Statistics, (in Theory and Applications of Special Functions, R.A. Askey Ed.) Academic Press, 1975, 497-520.

[29] L. JODAR, Explicit solutions for second order operator differential equations with two boundary value conditions, Linear Algebra Appl. 103 (1988), 73-86.

[30] L. JODAR and H. ABOU-KANDIL, A resolution method for Riccati differential systems coupled in their quadratic terms, SIAM J. Math. Anal. 19 (1988), 1225-1230.

[31] L. JODAR, Solving a class of generalized Lyapunov operator differential equations without the exponential operator function, Publicacions Matemàtiques, 34 (1990), 25-35.

[32] L. JODAR, R. COMPANY and E. NAVARRO, Laguerre matrix polynomials and systems of second-order differential equations, Applied Numer. Math. 15 (1994), 53-63.

FUNC. ESPECIALES Y EC. DIF. MATRICIALES 
[33] L. JODAR, R. COMPANY and E. PONSODA, Orthogonal matrix polynomials and systems of second order differential equations, Diff. Equations \& Dynam. Syst. 3, No.3 (1995), 269-288.

[34] L. JODAR and E. PONSODA, Computing continuous numerical solutions of matrix differential equations, Computers Math. Appl. 29, No.4 (1995), 73-84.

[35] L. JODAR and E. PONSODA, Non-autonomous Riccati-type matrix differential equations: existence interval, construction and continuous numerical solutions and error bounds, IMA J. Numer. Anal. 15 (1995), 61-74.

[36] L. JODAR and R. COMPANY, Hermite matrix polynomials and second order matrix differential equations, Approximation Theory Appl. 12, No.2 (1996), 20-30.

[37] L. JODAR, J.C. CORTES and J.L. MORERA, Construction and computation of variable coefficient Sylvester differential problems, Computers Math. Appl. 32, No.8 (1996), 41-50.

[38] L. JODAR and J.C. CORTES, Some properties of Gamma and Beta matrix functions, Applied Mathematics Letters (1997), (in print).

[39] L. JODAR and J.C. CORTES, Closed form general solution of the hypergeometric matrix differential equation, (in print).

[40] L. JODAR and J.C. CORTES, Rational matrix approximation with a priori error bounds for nonsymmetric matrix Riccati equations with analytic coefficients, (in print).

[41] T. KAILATH, Linear Systems, Prentice Hall, 1980.

[42] H.B. KELLER and M. LENTINI, Invariant imbedding, the box scheme and an equivalence between them, SIAM J. Numer. Anal.19 No.5 (1982), 942-962.

[43] S.S. KENNEY and R.B. LEIPNIK, Numerical integration of the differential matrix Riccati equation, IEEE Trans. Automatic Control, AC-30 (1985), 962-970.

FUNC. ESPECIALES Y EC. DIF. MATRICIALES 
[44] W. KRATZ, Quadratic Functionals in Variational Analysis and Control Theory, Akademie Verlag, Munchen, 1995.

[45] N.N. LÉBEDEV, Special Functions and Their Applications, Dover, New York, 1972.

[46] M. LEGUA, Ecuaciones Matriciales Diferenciales, en Diferencias y en Derivadas Parciales, Tesis Doctoral, Departamento de Matemática Aplicada, Universidad Politécnica de Valencia, 1991.

[47] Y.L. LUKE, The Special Functions and Their Approximations, Vols I and II, Academic Press, New York, 1969.

[48] M. MARITON, Jump Linear Systems in Automatic Control, Marcel Dekker, New York, 1990.

[49] R.J. MUIRHEAD, Systems of partial differential equations for hypergeometric functions of matrix argument, Ann. Math. Statist. 41 (1970), 991-1001.

[50] J.M. ORTEGA and W.C. RHEINBOLDT, Iterative Solution of Nonlinear Equations in Several Variables, Academic Press, New York, 1970.

[51] Y. OSHMAN and I. Y. BAR-ITZHACK, Eigenfactor solution of the matrix Riccati equation. A continuous square root algorithm, Proc. 23rd Conf. on Decision and Control, (Las Vegas, 1984), 503-508.

[52] E.D. RAINVILLE, Special Functions, Chelsea, New York, 1960.

[53] W. T. REID, Riccati Differential Equations, Academic Press, New York, 1972.

[54] K. REKTORYS, The Method of Discretization in Time and Partial Differential Equations, Reidel, Dordrecht, 1982.

[55] W. RUDIN, Principles of Mathematical Analysis, McGraw-Hill, New York, 1964.

[56] S. SAKS and A. ZYGMUND, Analytic Functions, Elsevier, Amsterdam, 1971.

FUNC. ESPECIALES Y EC. DIF. MATRICIALES 
[57] J. SCHIFF and S. SHNIDER, A natural approach to the numerical integration of Riccati differential equations (Tech. Rep. Bar Ilan Univ. Israel, July 1996).

[58] S.M. SERBIN and C.A. SERBIN, A time-stepping procedure for $X^{\prime}=A_{1} X+X A_{2}+D ; \quad X(0)=C$, IEEE Trans. Automatic Control, Vol. 25 (1980), 1138-1141.

[59] A. W. STARR and Y. C. HO, Non-zero sum differential games, J. Optim. Theory Appl. 3 (1969), 179-197.

[60] M. SUBRAHMANYAM, On a numerical method of solving the Lyapunov and Sylvester equations, Int. J. Control, Vol. 43 (1986), 433-439.

[61] D.D. SWORDER, Control of a linear system with non-markovian modal changes, J. Economic Dynamics and Control, 2 (1980), 233-240.

[62] N.M. TEMME, Special Functions, John-Wiley \& Sons, New York, 1996.

[63] E.C. TITCHMARSH, The Theory of Functions, Oxford Univ. Press, Oxford, 1993.

[64] A. ZANNA, The method of iterated commutators for ordinary differential equations on Lie groups, Tech. Rep. DAMPT 1996/NA12, Univ. of Cambridge. 


\title{
PUBLICACIONES EN REVISTAS INTERNACIONALES DE MATEMÁTICAS \\ INDEXADAS EN JCR-SCI-ISI DERIVADAS DE ESTA TESIS
}

\author{
AUTORES: L. Jódar, J.C. Cortés, J.L. Morera \\ TÍTULO: Construction and computation of variable coefficient Sylvester differential \\ problems (doi:10.1016/0898-1221(96)00165-4) \\ REVISTA: Comput. Math. Appl. (ISSN: 0898-1221) \\ VOLUMEN/PÁGINAS/EDITORIAL/AÑO: vol. 32 (8)/41-50/Pergamon/1996 \\ CLAVE: A
}

AUTORES: L. Jódar, J.C. Cortés

TÍTULO: Some properties of gamma and beta matrix functions (doi:10.1016/S08939659(97)00139-0)

REVISTA: Appl. Math. Lett. (ISSN: 0893-9659)

VOLUMEN/PÁGINAS/EDITORIAL/AÑO: vol. 11 (1)/89-93/Pergamon/1998

CLAVE: A

AUTORES: L. Jódar, J.C. Cortés

TÍTULO: Rational matrix approximation with a priori error bounds for nonsymmetric matrix Riccati equations with analytic coefficients

REVISTA: IMA J. Numer. Anal. (ISSN: 0272-4979)

VOLUMEN/PÁGINAS/EDITORIAL/AÑO: vol. 18 (4)/545-561/Oxford University

Press/1998

CLAVE: A

AUTORES: L. Jódar, J.C. Cortés

TÍTULO: On the hypergeometric matrix function (doi:10.1016/S0377-0427(98)00158-

7)

REVISTA: J. Comput. Appl. Math. (ISSN: 0377-0427)

VOLUMEN/PÁGINAS/EDITORIAL/AÑO: vol. 99 (1-2)/205-217/Elsevier/1998

CLAVE: A

AUTORES: L. Jódar, J.C. Cortés

TÍTULO: Closed form general solution of the hypergeometric matrix differential equation (doi:10.1016/S0895-7177(00)00187-4)

REVISTA: Math. Comput. Model. (ISSN: 0895-7177)

VOLUMEN/PÁGINAS/EDITORIAL/AÑO: vol. 32 (9)/1017-1028/Pergamon/2000

CLAVE: A 